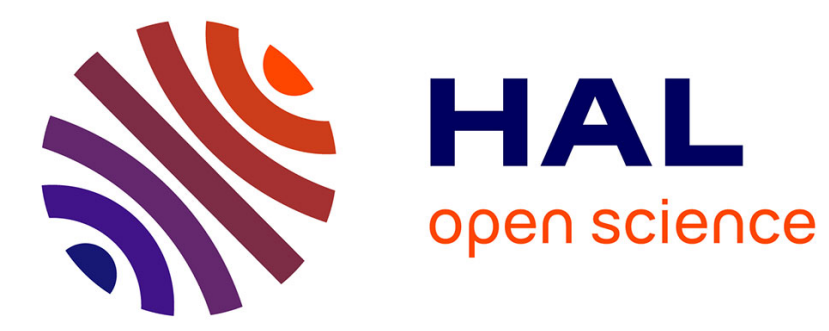

\title{
Equation of state for dense nucleonic matter from metamodeling. I. Foundational aspects
}

Jérôme Margueron, Rudiney Hoffmann Casali, Francesca Gulminelli

\section{To cite this version:}

Jérôme Margueron, Rudiney Hoffmann Casali, Francesca Gulminelli. Equation of state for dense nucleonic matter from metamodeling. I. Foundational aspects. Phys.Rev.C, 2018, 97 (2), pp.025805. 10.1103/PhysRevC.97.025805 . hal-01724996

\section{HAL Id: hal-01724996 https://hal.science/hal-01724996}

Submitted on 16 Mar 2020

HAL is a multi-disciplinary open access archive for the deposit and dissemination of scientific research documents, whether they are published or not. The documents may come from teaching and research institutions in France or abroad, or from public or private research centers.
L'archive ouverte pluridisciplinaire HAL, est destinée au dépôt et à la diffusion de documents scientifiques de niveau recherche, publiés ou non, émanant des établissements d'enseignement et de recherche français ou étrangers, des laboratoires publics ou privés. 


\title{
Equation of state for dense nucleonic matter from metamodeling. I. Foundational aspects
}

\author{
Jérôme Margueron, ${ }^{1,2}$ Rudiney Hoffmann Casali, ${ }^{2,3}$ and Francesca Gulminelli ${ }^{4}$ \\ ${ }^{1}$ Institute for Nuclear Theory, University of Washington, Seattle, Washington 98195, USA \\ ${ }^{2}$ Institut de Physique Nucléaire de Lyon, CNRS/IN2P3, Université de Lyon, Université Claude Bernard Lyon 1, \\ F-69622 Villeurbanne Cedex, France \\ ${ }^{3}$ Departamento de Física, Instituto Tecnológico de Aeronáutica, CTA, 12228900, São José dos Campos, São Paulo, Brazil \\ ${ }^{4}$ CNRS, ENSICAEN, UMR6534, LPC, F-14050 Caen Cedex, France
}

(Received 23 August 2017; revised manuscript received 21 November 2017; published 28 February 2018)

\begin{abstract}
Metamodeling for the nucleonic equation of state (EOS), inspired from a Taylor expansion around the saturation density of symmetric nuclear matter, is proposed and parameterized in terms of the empirical parameters. The present knowledge of nuclear empirical parameters is first reviewed in order to estimate their average values and associated uncertainties, and thus defining the parameter space of the metamodeling. They are divided into isoscalar and isovector types, and ordered according to their power in the density expansion. The goodness of the metamodeling is analyzed against the predictions of the original models. In addition, since no correlation among the empirical parameters is assumed a priori, all arbitrary density dependences can be explored, which might not be accessible in existing functionals. Spurious correlations due to the assumed functional form are also removed. This meta-EOS allows direct relations between the uncertainties on the empirical parameters and the density dependence of the nuclear equation of state and its derivatives, and the mapping between the two can be done with standard Bayesian techniques. A sensitivity analysis shows that the more influential empirical parameters are the isovector parameters $L_{\text {sym }}$ and $K_{\text {sym }}$, and that laboratory constraints at supersaturation densities are essential to reduce the present uncertainties. The present metamodeling for the EOS for nuclear matter is proposed for further applications in neutron stars and supernova matter.
\end{abstract}

DOI: 10.1103/PhysRevC.97.025805

\section{INTRODUCTION}

Since the discovery of neutron stars (NS) in 1967 [1-3], the accurate prediction of the nuclear equation of state (EOS) has become of great importance, and a lot of effort, both from the theoretical and the experimental sides, has been devoted to this aim. The seminal work of Tolman et al. in 1939 had proved that considering only the kinetic contribution of nucleons to nuclear matter equation of state provides a limit in the maximum mass of neutron stars of about $0.7 \mathrm{M}_{\odot}[4,5]$. This contradicts the present observations for the canonical NS mass, which is of the order of $1.44 \mathrm{M}_{\odot}[6]$, as well as the recent observations proving the existence of about $2 \mathrm{M}_{\odot}$ NS $[7,8]$. These observational data clearly demonstrate the importance of the nuclear interaction for understanding the global properties of neutron stars.

Several ab initio approaches have been developed for the accurate prediction of NS equation of state; see, for instance, Refs. $[9,10]$ for recent reviews. More recently, new nuclear potentials (chiral effective field theory (EFT)) have been developed, offering the possibility of performing calculations in perturbation theory $[11,12]$, and they have been implemented as well in quantum Monte Carlo (QMC) methods [13-15]. These potentials have also been applied to the NS EOS; see, for instance, Refs. $[16,17]$. While there is a convergence in the prediction of these EOS at low density, such method might fail above the saturation density of nuclear matter because an expansion in supposedly small parameters is no longer really valid there. In addition, there are larger deviations between the different predictions above saturation density, mainly because of the different treatments of the many-body correlations and the different nuclear interactions; see, for instance, Ref. [18] for a detailed comparison of some of these approaches.

With the development of $\mathrm{x}$-ray observations of the thermal emission from the surface of neutron stars, it was envisioned that the nuclear EOS may be directly determined from observational data such as NS radii [19-30]. In all these papers, the nuclear EOS is expressed in terms of a reduced number of parameters, such as matching densities of piecewise polytropes first introduced in Ref. [19]. The use of polytropes, while extremely simple and not too far from the model predictions, does not allow a simple connection to the present nuclear physics knowledge, such as nuclear saturation and empirical parameters, nor can it bring information concerning matter composition, such as the proton fraction. It is therefore interesting to extend these ideas toward a more complementary approach between astrophysical and nuclear experimental constraints.

Other approaches for the nuclear EOS are derived from some simple nuclear interaction, such as Skyrme-type contact interactions complemented by a density-dependent term [16,31]. While extremely useful and simple, the densitydependent term usually brings correlations among the nuclear empirical parameters which may be unphysical [32,33]. Nonrelativistic Skyrme-type EOS [34,35] as well as relativistic ones $[36,37]$ can be selected according to their ability to reproduce $a b$ initio calculations. 
A third model of the nuclear EOS is based on a Taylor expansion of the nuclear EOS around saturation density [22] or a Fermi momentum expansion [38]. This kind of approach offers a unique possibility to incorporate in the nuclear EOS the best knowledge issued from nuclear physics, reducing the number of free parameters. The Taylor expansion allows the separation of the low-order derivatives, which are better determined by nuclear experiments, from the high-order ones, which are best determined by NS observations. Indeed, the higher order parameters are more sensitive to the EOS at the highest densities, which are difficult to access from nuclear laboratory experiments.

In this paper, a metamodel, or a "model of a model" [39], for the nucleonic EOS is proposed and analyzed. Metamodels are a practical solution to solving complex and numerical issues and/or facilitating optimization under uncertainty. They are therefore often used to provide fast approximations to the results of more complex problems, and to perform comparative analysis of different models belonging to the class covered by the metamodeling. Conceptually, metamodels build a hypersurface from a limited amount of input and output data and approximate the output over a much wider parameter space; see Refs. [40-42] for an overview of metamodeling techniques. Metamodels have to be evaluated with respect to the goodness and there is no proof of existence or of uniqueness in general. A metamodel is always associated to a given model or class of models. In the present application, we will consider homogeneous nucleonic EOS. In principle, different metamodels can be introduced to represent different model classes, e.g., nucleonic EOS against high-density phase transition EOS. The goodness of the data adjustment with respect to one of these classes can, for instance, be analyzed by introducing Bayesian factors [39]. We introduce the concept of a metamodel for the nucleonic EOS since it present several interesting advantages: (i) It provides a unique mapping of very different existing EOS with many different input parameters, (ii) it provides a flexible approach that can interpolate continuously between existing EOS, (iii) as a consequence, it may orientate the preferred input parameters toward values which are not among the existing EOS, (iv) it allows the definition of a generic model where the nuclear physics knowledge acquired from laboratory experiments can be simply encoded as input parameters, (v) it includes in its parameter space the results of complex $a b$ initio models, and can thus be used to extract the constraints on the EOS imposed by them, (vi) and finally, combined with the Bayesian framework, it facilitates the estimation of the experimental and theoretical error bars into confidence levels for the astrophysics observables. In this paper, we introduce and analyze the properties of this nucleonic metamodeling, while the connection with NS observables is performed in a second paper [43]. Further extensions of this approach to the description of nonhomogeneous matter and/or of dense matter phase transitions can easily be developed in the future from the present framework.

The present paper is organized as follows: In Sec. II, a review of the experimental information on the nuclear empirical parameters is performed, and their uncertainties are estimated. To this aim, predictions from relativistic and nonrelativistic, phenomenological and ab initio interactions are compiled and compared, and uncertainties are obtained from a statistical analysis. The metamodeling is formulated in Sec. III, presenting different options for the Taylor expansion. The quality of the different strategies is estimated by comparing the convergence of predictions with respect to a reference EOS. Section IV explores the flexibility of the meta-EOS. We show that this metamodeling can very accurately reproduce a large number of existing EOS, and at the same time it can explore density dependences which are not accessible to usual phenomenological functionals because of the imposed functional form. In that section, it is also shown that the huge uncertainty in higher order empirical parameters can only be reduced if extra empirical information is added on a second higher density reference point, in addition to the saturation density. One of the advantages of the present meta-EOS is the fact that no a priori correlations are imposed on the empirical parameters. The physical correlations can be added $a$ posteriori as illustrated in the second paper [43]. We perform a sensitivity analysis of the meta-EOS to the different empirical parameters by varying them one by one according to their uncertainties. This is done in Sec. V, where we show that the most influential parameters are the isovector ones, namely $L_{\text {sym }}, K_{\text {sym }}$, and $Q_{\text {sym }}$. This stresses once again the need for experimental constraints at high density on asymmetric matter, typically from high-energy heavy-ion collisions with rare isotopic beams. Finally, conclusions and outlooks are presented in Sec. VI.

\section{EMPIRICAL CHARACTERIZATION OF THE NUCLEAR EQUATION OF STATE}

In the following, we analyze the properties of nuclear matter composed of neutrons and protons with different isoscalar (is) density $n_{0}=n_{n}+n_{p}$ and isovector (iv) density $n_{1}=n_{n}-n_{p}$, where $n_{n / p}$ is the neutron/proton density defined as

$$
n_{n / p}=\frac{1}{3 \pi^{2}} k_{F_{n / p}}^{3},
$$

where $k_{F_{n / p}}$ is the neutron/proton Fermi energy. Isospin asymmetric nuclear matter (ANM) can also be defined in terms of the asymmetry parameter $\delta=n_{1} / n_{0}$. The two boundaries $\delta=0$ and 1 correspond to symmetric nuclear matter (SNM) and to pure neutron matter (PNM). The saturation density of SNM is defined as the density at which the symmetric matter pressure is zero and it is denoted as $n_{\text {sat }}$.

The general properties of relativistic and nonrelativistic nuclear interactions are often characterized in terms of the nuclear empirical parameters, defined as the coefficients of the following series expansion in the parameter $x=\left(n_{0}-n_{\text {sat }}\right) /$ $\left(3 n_{\text {sat }}\right)[44]$,

$$
\begin{aligned}
e_{\mathrm{is}}= & E_{\mathrm{sat}}+\frac{1}{2} K_{\mathrm{sat}} x^{2}+\frac{1}{3 !} Q_{\mathrm{sat}} x^{3}+\frac{1}{4 !} Z_{\mathrm{sat}} x^{4}+\cdots, \\
e_{\mathrm{iv}}= & E_{\mathrm{sym}}+L_{\mathrm{sym}} x+\frac{1}{2} K_{\mathrm{sym}} x^{2} \\
& +\frac{1}{3 !} Q_{\mathrm{sym}} x^{3}+\frac{1}{4 !} Z_{\mathrm{sym}} x^{4}+\cdots,
\end{aligned}
$$

where the isoscalar energy $e_{\text {is }}$ and the isovector energy $e_{\mathrm{iv}}$ enter into the definition of the energy per nucleon in nuclear matter, 
defined as

$$
e\left(n_{0}, n_{1}\right)=e_{\mathrm{is}}\left(n_{0}\right)+\delta^{2} e_{\mathrm{iv}}\left(n_{0}\right) .
$$

The isovector energy $e_{\mathrm{iv}}$ is often called the symmetry energy $S\left(n_{0}\right)=e_{\mathrm{iv}}\left(n_{0}\right)$. Note that this definition implies a parabolic approximation for the isospin dependence, while the proper definition is given by the second derivative with respect to $\delta$ around symmetric matter; see Eq. (12) below.

The empirical parameters entering the series expansion (2) and (3) are separated into two channels [18,45]: the isoscalar channel which defines the saturation energy $E_{\text {sat }}$, the saturation density $n_{\text {sat }}$, the incompressibility modulus $K_{\text {sat }}$, the isoscalar skewness $Q_{\text {sat }}$, and the isoscalar kurtosis $Z_{\text {sat }}$; and the isovector channel which defines the symmetry energy $E_{\text {sym }}$, the slope $L_{\text {sym }}$, the isovector incompressibility $K_{\text {sym }}$, the isovector skewness $Q_{\text {sym }}$, and the isovector kurtosis $Z_{\text {sym }}$. There is no unique nomenclature for the empirical parameters, but in principle, Eqs. (2) and (3) make ours unambiguous. A very clear synthesis of the various terminologies used in the literature is discussed in the appendix of Ref. [44].

The energy per nucleon (4) can be expressed in the following compact form $[45,46]$,

$$
e\left(n_{0}, n_{1}\right)=\sum_{\alpha \geqslant 0} \frac{1}{\alpha !}\left(c_{\alpha}^{\mathrm{is}}+c_{\alpha}^{\mathrm{iv}} \delta^{2}\right) x^{\alpha},
$$

where the coefficients $c_{\alpha}^{\text {is/iv }}$ are the empirical parameters introduced in Eqs. (2) and (3) [44]. Note that the coefficient $c_{1}^{\text {is }}=0$ is due to the choice of the saturation density $n_{\text {sat }}$ as the reference density in the definition of $x$. Consequently, choosing an arbitrary density as reference in the definition of $x$ would lead to a nonvanishing $c_{1}^{\text {is }}$, and $n_{\text {sat }}$ would be determined by the isoscalar empirical parameters. The total number of free parameters is thus conserved: Considering $c_{1}^{\text {is }}$ or $n_{\text {sat }}$ as isoscalar empirical parameter for $\alpha=1$, it is two per exponent $\alpha$.

The empirical properties are determined from nuclear physics experiments such as measurements of nuclear masses, charge-density profiles, and analysis of collective modes (ISGMR, IVGDR, etc.). More details of the experimental determinations of the empirical parameters are presented in Sec. II A.

The series expansion (5) in the parameter $x$ is in principle infinite, and it is not guaranteed that this expansion converges. The convergence property is analyzed in Sec. III, however, and anticipating the results, it is shown that in a density range going up to $4 n_{\text {sat }}$ an order by order convergence for the binding energy, the pressure and the sound velocity are found. This result is tested for a large number of nuclear interactions in Sec. IV. In this section, we therefore concentrate on the experimental determination of the first terms in the expansion (5).

The expansion in the asymmetry parameter $\delta$ in Eq. (5) does not include terms beyond second order in $\delta$. Note, however, that small corrections may appear, such as those induced by the $T=0$ pairing or quarteting $[47,48]$, which have been considered in recent works; see, for instance, Refs. [38,49-51]. $A b$ initio approaches show that the energy per nucleon in homogeneous asymmetric nuclear matter is mostly quadratic in $\delta$ [52,53], and residual nonquadraticities are mostly related to the kinetic energy part of the total energy (including the effective mass splitting) [46]. This is also confirmed by an analysis of various finite-range nuclear forces [54]. For this reason, in Sec. III we will replace the global expansion (4) by an expression where the contribution of the kinetic energy is expressed separately, and limit the parabolic approximation to the interaction part.

In the following, we first review the "experimental" determination of the first parameters in Eq. (5), hereafter called "low order." In Sec. II A, we list a large, but certainly not extensive, amount of referenced analyses where authors have optimized their models on specific experimental data to extract some of the empirical parameters. We call these analyses "experimental" in opposition to the generic determination which is presented in Sec. IIB. In the generic approach, the parameters are directly deduced from a set of models known by their ability to reasonably predict a large number of nuclear properties, such as masses and radii at least. The generic approach is supposed to provide an upper bound on the empirical parameters uncertainties. For the low-order empirical parameters, a good overlap is found between the "experimental" analysis and the generic one. The advantage of the generic analysis is that it could also provide an estimation of the uncertainties associated with the high-order empirical parameters which are yet quite unknown.

\section{A. Experimental determination of the nuclear empirical parameters}

There is a very important experimental and theoretical program aiming at a better estimation of the nuclear empirical parameters. For this reason, some of the empirical quantities are rather well determined. This concerns essentially the first terms of the series expansion (5), such as the saturation energy, the saturation density, the incompressibility modulus, and the symmetry energy. We have grouped them in the so-called group A and presented them in Table I. The other empirical parameters are less well known, and we will show that this second group of nuclear empirical parameters can be divided into two subgroups: the one for which we can give a range of variation compatible with our experimental knowledge, the so-called group B shown in Table II, and a group of parameters which are yet quite undetermined and not presently accessible by nuclear experiments, the so-called group C. In the following, we review the experimental determination of the empirical parameters for the groups A and B. Let us, however, notice that the following review is not exhaustive but more illustrative. The aim of the subsection is to justify the current estimation of these empirical parameters.

The values reported in Table I are extracted from experimental analysis and can therefore be considered as closely related to nuclear data. They are not directly determined from experimental data since these quantities are not accessible to experimental probes without the use of a theoretical model. For instance, the saturation density is extrapolated from fits of finite nuclei density profiles. An additional difficulty comes from the fact that the isoscalar density is not directly measurable from electron scattering in finite nuclei, and the relation between 
TABLE I. Group A: saturation energy $E_{\text {sat }}$, density $n_{\text {sat }}$, incompressibility $K_{\text {sat }}$, and symmetry energy $E_{\text {sym }}$ estimated from various analyses of experimental data. See text for more details.

\begin{tabular}{|c|c|c|c|c|c|}
\hline El. scatt. & Wang-99 [55] & & 0.1607 & $\begin{array}{l}235 \\
\pm 15\end{array}$ & \\
\hline LDM & Myers-66 [56] & -15.677 & $0.136^{\mathrm{a}}$ & 295 & 28.06 \\
\hline LDM & Royer-08 [57] & -15.5704 & $0.133^{\mathrm{a}}$ & & 23.45 \\
\hline LSD & Pomorski-03 [58] & -15.492 & $0.142^{\mathrm{a}}$ & & 28.82 \\
\hline & & & \pm 0.004 & & \\
\hline INM & Satpathy-99 [61] & -16.108 & 0.1620 & $\begin{array}{l}288 \\
\pm 20\end{array}$ & \\
\hline DF-Skyrme & Tondeur-86 [62] & & 0.158 & & \\
\hline DF-Skyrme & Klupfel-09 [63] & $\begin{array}{r}-15.91 \\
\pm 0.06\end{array}$ & $\begin{array}{r}0.1610 \\
\pm 0.0013\end{array}$ & $\begin{array}{c}222 \\
\pm 8\end{array}$ & $\begin{array}{r}30.7 \\
\pm 1.4\end{array}$ \\
\hline DF-Skyrme & McDonnell-15 [66] & $\begin{array}{r}-15.75 \\
\pm 0.25\end{array}$ & $\begin{array}{r}0.160 \\
\pm 0.005\end{array}$ & $\begin{array}{l}220 \\
\pm 20\end{array}$ & $\begin{array}{l}29 \\
\pm 1\end{array}$ \\
\hline DF-NLRMF & $\mathrm{NL}^{*}[67]$ & -16.3 & 0.15 & 258 & 38.7 \\
\hline DF-NLRMF & PK [68] & -16.27 & 0.148 & 283 & 37.7 \\
\hline DF-DDRMF & DDME1,2 [69,70] & $\begin{array}{r}-16.17 \\
\pm 0.03\end{array}$ & $\begin{aligned} & 0.152 \\
\pm & 0.00\end{aligned}$ & $\begin{array}{c}247 \\
\pm 3\end{array}$ & $\begin{array}{r}32.7 \\
\pm 0.4\end{array}$ \\
\hline DF-DDRMF & PK [68] & 16.27 & 0.150 & 262 & 36.8 \\
\hline $\begin{array}{l}\text { Present } \\
\text { Estimation }\end{array}$ & & $\begin{array}{r}-15.8 \\
\pm 0.3\end{array}$ & $\begin{array}{r}0.155 \\
\pm 0.005\end{array}$ & $\begin{array}{l}230 \\
\pm 20\end{array}$ & $\begin{array}{l}32 \\
\pm 2\end{array}$ \\
\hline
\end{tabular}

a Value determined from $r_{0}$.

the charge density and the total density is thus performed via a theoretical model. The neutron density can be determined in a relatively model-independent way by measurement of the parity-violating electron scattering asymmetry from ${ }^{208} \mathrm{~Pb}$. This is the aim of the PREX experiment at Jefferson Laboratory [80].

The values for the saturation energy reported in Table I are remarkably stable in the different analysis. From Table I, the current value of $E_{\text {sat }}$ is estimated to be $-15.8 \pm 0.3 \mathrm{MeV}$. Let us mention a recent estimation of $E_{\text {sat }}$ and its uncertainty based on liquid drop models (LDM) and the frequency-domain bootstrap method [81]. The obtained value is $-15.56 \pm 0.17 \mathrm{MeV}$, which is slightly lower, but still compatible with our current estimation.

The saturation density is more difficult to determine from the analysis presented in Table I. The value estimated from LDM is lower than the one obtained from density functional (DF) models, which are supposed to provide the more accurate determination of the saturation density. This is confirmed by the fact that the values extracted from the droplet model (DM) and the finite-range droplet model (FRDM), which are more realistic than the original LDM [59], are closer to the ones extracted by DF. We have selected the DF models for which the value for the saturation density was not assumed a priori in the fitting protocol to global properties of finite nuclei such as binding energies and charge radii. The value obtained for the saturation density could therefore be considered as a prediction of these models. In summary, we consider the following current estimation of $n_{\text {sat }}=0.155 \pm 0.005 \mathrm{fm}^{-3}$. Note that the error in the determination of these quantities was larger some decades ago; see, for instance [82].

The incompressibility modulus $K_{\text {sat }}$ given in Table I varies from 210 up to $300 \mathrm{MeV}$, revealing here also the difficulty in estimating this quantity from experimental data as well as its model dependence. A more systematical review of the various theoretical predictions for $K_{\text {sat }}$ is presented in Ref. [83]. The determination of the incompressibility modulus from the LDM is usually not very accurate $[83,84]$. A better determination can be obtained from a method proposed by Blaizot $[84,85]$, based on the correlation between the isoscalar giant monopole resonance (ISGMR) energy and the empirical parameter $K_{\text {sat }}$. This estimation remains quite model dependent, and for instance, a lower value $K_{\text {sat }} \approx 210 \mathrm{MeV}$ is obtained for the BCP functional [86] and Gogny interactions [84], while a higher value $K_{\text {sat }} \approx 250-270 \mathrm{MeV}$ is predicted from relativistic mean field (RMF) approaches [87-89]. A part of this model dependence can be understood from the violations of self-consistency in some early calculations [90]. This model dependence might also reveal a more complex correlation in terms of several empirical parameters, instead of the single one proposed by Blaizot. It was indeed shown that the ISGMR is also sensitive to symmetry properties, and information on $K_{\text {sat }}$ cannot be easily deconvoluted from information on $K_{\text {sym }}$ [91]. For a deeper review, see Ref. [92]. It was also recently shown that higher 
TABLE II. Group B parameters: isoscalar skewness $Q_{\text {sat }}$, slope of the symmetry energy $L_{\text {sym }}$, and isovector incompressibility $K_{\text {sym }}$. The parameter $K_{\tau}$ is defined as $K_{\tau}=K_{\text {sym }}-6 L_{\text {sym }}-Q_{\text {sat }} L_{\text {sym }} / K_{\text {sat }}$. See text for more details.

\begin{tabular}{|c|c|c|c|c|c|}
\hline Model & Ref. & $\begin{array}{c}Q_{\text {sat }} \\
(\mathrm{MeV})\end{array}$ & $\begin{array}{c}L_{\text {sym }} \\
(\mathrm{MeV})\end{array}$ & $\begin{array}{c}K_{\text {sym }} \\
(\mathrm{MeV})\end{array}$ & $\begin{array}{c}K_{\tau} \\
(\mathrm{MeV})\end{array}$ \\
\hline DF-Skyrme & Berdichevsky-88 [71] & 30 & 0 & & \\
\hline DF-Skyrme & Farine-97 [72] & $\begin{array}{l}-700 \\
\pm 500\end{array}$ & & & \\
\hline DF-Skyrme & Alam-14 [31] & $\begin{array}{l}-344 \\
\pm 46\end{array}$ & $\begin{array}{c}65 \\
\pm 14\end{array}$ & $\begin{array}{l}-23 \\
\pm 73\end{array}$ & $\begin{array}{c}-322 \\
\pm 34\end{array}$ \\
\hline DF-Skyrme & McDonnell-15 [66] & & $\begin{array}{c}40 \\
\pm 20\end{array}$ & & \\
\hline DF-NLRMF & NL3* [67] & 124 & 123 & 106 & -690 \\
\hline DF-NLRMF & PK [68] & -25 & 116 & 55 & -630 \\
\hline DF-DDRMF & DDME1,2 [69,70] & $\begin{array}{l}400 \\
\pm 80\end{array}$ & $\begin{array}{l}53 \\
\pm 3\end{array}$ & $\begin{array}{l}-94 \\
\pm 7\end{array}$ & $\begin{array}{l}-500 \\
\pm 7\end{array}$ \\
\hline DF-DDRMF & PK [68] & -119 & 79.5 & -50 & -491 \\
\hline Correlation & Centelles-09 [73] & & $\begin{array}{c}70 \\
\pm 40\end{array}$ & & $\begin{array}{l}-425 \\
\pm 175\end{array}$ \\
\hline DF-RPA & Carbone-10 [74] & & $\begin{array}{c}60 \\
\pm 30\end{array}$ & & \\
\hline Correlation & Danielewicz-14 [75] & & $\begin{array}{c}53 \\
\pm 20\end{array}$ & & \\
\hline Correlation & Newton-14 [76] & & $\begin{array}{c}70 \\
\pm 40\end{array}$ & & \\
\hline Correlation & Lattimer-14 [77] & & $\begin{array}{c}53 \\
\pm 20\end{array}$ & & \\
\hline GMR & Sagawa-07 [78] & & & & $\begin{array}{c}-500 \\
\pm 50\end{array}$ \\
\hline GMR & Patel-14 [79] & & & & $\begin{array}{l}-550 \\
\pm 100\end{array}$ \\
\hline Present & & 300 & 60 & -100 & -400 \\
\hline Estimation & & \pm 400 & \pm 15 & \pm 100 & \pm 100 \\
\hline
\end{tabular}

order isoscalar parameters also play a role, and the correlation analysis should be performed in terms of several empirical parameters instead of only one, such as $K_{\text {sat }}$ and $Q_{\text {sat }}[32,33]$. The value of $Q_{\text {sat }}$ is yet undetermined, and most of the model dependence in the determination of $K_{\text {sat }}$ can be attributed to the uncertainties in $Q_{\text {sat }}$ [33]. In other words, a better estimation of $Q_{\text {sat }}$ would refine the estimation of $K_{\text {sat }}$ based on the correlation with the ISGMR. From a LDM approach separating the bulk contribution $\left(K_{\text {sat }}\right)$ from the surface one (largely influenced by $Q_{\text {sat }}$ ), the importance of the surface properties for the determination of $K_{\text {sat }}$ was pointed out as well [83]. An estimation of $K_{\text {sat }}=230 \pm 40 \mathrm{MeV}$ was given in Refs. [32,33] where the error bar contains the maximum and minimum possible values for $K_{\text {sat }}$. It is therefore larger than a $1 \sigma$ uncertainty, where $1 \sigma$ is the error bar accounting for $68 \%$ of the models around the centroid. In summary, the current estimation of $K_{\text {sat }}$ can be given as $230 \pm 20 \mathrm{MeV}$, where the error bar corresponds to $1 \sigma$ uncertainty.

It is interesting to observe the correlations between the empirical parameters $K_{\text {sat }}$ and $Q_{\text {sat }}$ represented in Fig. 1 . This correlation is shown for Skyrme models (purple line), RMF models (light-green area), RHF models (light-blue area),

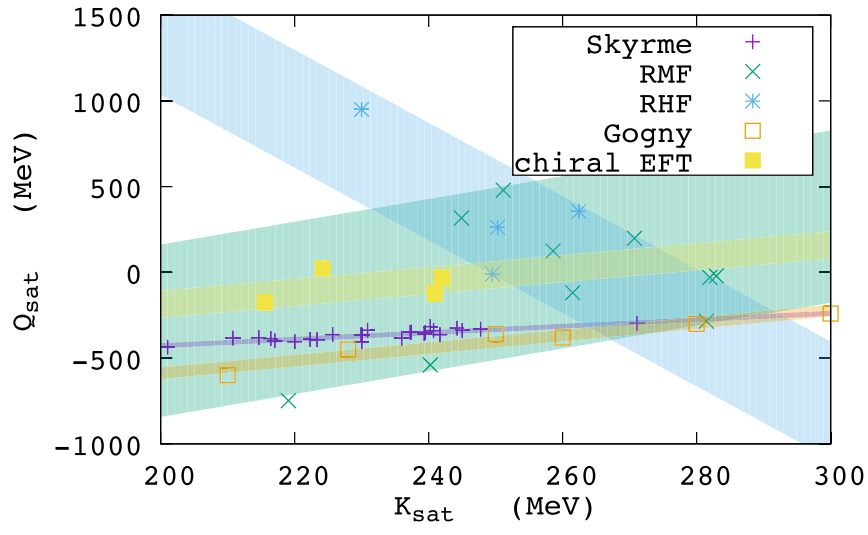

FIG. 1. Correlation between the empirical parameters $K_{\text {sat }}$ and $Q_{\text {sat }}$ for different kind of nuclear interactions: Skyrme, Gogny, RMF, and relativistic Hartree-Fock (RHF). Points from EFT approach are also plotted. The points are obtained from Tables IX-XII, except for the Gogny model, which is extracted from Ref. [33], and the colored bands come from fits of the data including their dispersion considering $67 \%$ of the best models.

Gogny models (orange line), and chiral EFT predictions (yellow line). The correlation bands for each models are shown for clarity. They are obtained assuming a linear correlation between the values of $K_{\text {sat }}$ and $Q_{\text {sat }}$, and the width of the bands are determined from the $1-\sigma$ deviation. The strongest correlation is found for the Skyrme and Gogny models, already suggested in Ref. [33], and the origin of this correlation can be found in the so-called $t_{3}$ density-dependent terms which dominate in $K_{\text {sat }}$ and $Q_{\text {sat }}$. It is, however, interesting to remark that also the relativistic models (RMF and RHF) exhibit a correlation between these empirical parameters, even if its origin is less easy to analyze. In addition, the very different correlations between the various kinds of models shown in Fig. 1 indicate a strong model dependence of the correlation that might not reflect a physical property. Since $K_{\text {sat }}$ and $Q_{\text {sat }}$ govern the density dependence of the equation of state in symmetric matter (SM) and around saturation density, the correlation shown in Fig. 1 indicates that models do not explore all possible density dependences.

This is one of the main motivations of the present work: In the following Sec. V, we propose a metamodeling which can explore the full parameter space (including $K_{\text {sat }}$ and $Q_{\text {sat }}$ ), with no a priori restriction. Physical correlations could be added by imposing some constraints to the metamodeling, as illustrated in the second paper [43].

While the binding energies $E_{\text {sat }}$ are predicted in a quite narrow interval for the various models presented in Table I, the symmetry energy varies substantially among LDM, DM, and DF models. This might be because the value for the symmetry energy is very strongly related to the value of the slope of the symmetry energy $L_{\text {sym }}$ in many models $[74,75,93]$. The quantity which matters in the fit to experimental energies seems to be more closely related to the symmetry energy at the average density of nuclei, at around $(2 / 3) n_{\text {sat }}$ [94]. In addition, it has also been observed that RMF models prefer large values for the symmetry energy, such as $34-36 \mathrm{MeV}$, and it has been proposed that the symmetry energy and the 
incompressibility modulus $K_{\text {sat }}$ are correlated in DF models [91]. Furthermore, a recent analysis of the bulk and surface contributions of the symmetry energy have shown that the sign of surface contribution depends strongly on the choice for the asymmetry parameter: the global asymmetry parameter or the bulk asymmetry parameter, which contains a correction from the neutron skin [95]. Considering this large model dependence, the current estimation of $E_{\mathrm{sym}}$ is approximately $32 \pm 2 \mathrm{MeV}$ and this is in agreement with other estimations; see Refs. [96-99].

We now discuss the parameters of group B given in Table II: $Q_{\text {sat }}, L_{\text {sym }}$, and $K_{\text {sym }}$. These parameters are not yet very well determined, but a better accuracy might be reached in the near future. We first discuss the skewness parameter $Q_{\text {sat }}$. This parameter is poorly known and there are very few experimental analyses which propose an estimation. An analysis of charge and mass radii of tin isotopes concluded that either $Q_{\text {sat }} \approx 30 \mathrm{MeV}$ or $L_{\text {sym }} \approx 0 \mathrm{MeV}$ [71]. Another analysis of the incompressibility modulus concluded that $Q_{\text {sat }} \approx-700 \pm$ $500 \mathrm{MeV}$ [72]. This very large error bar reflects once again the model dependence of $Q_{\text {sat }}$, induced by its correlation with the incompressibility modulus, as shown in Fig. 1. It is therefore very difficult to estimate the value of this parameter and in the following, we shall explore a large domain.

The parameter $L_{\text {sym }}$ is much discussed nowadays and a large number of experiments aim at determining its value [100]. Combining different constraints from neutron skin thickness, heavy-ion collisions, dipole polarizability, nuclear masses, giant-dipole resonances, and isobaric analog states, it was recently concluded that the value of $L_{\mathrm{sym}}$ should be between 33 and $72 \mathrm{MeV}$ [77,101]. Note that in Ref. [77] the symmetry energy is comprised between 31 and $36 \mathrm{MeV}$, which is consistent with the present estimation given in Table I. Other analyses predict slightly larger values for $L_{\text {sym }}$, and integrating all analyses, we come to the following estimation: $L_{\text {sym }}=60 \pm 15 \mathrm{MeV}$.

The isospin dependence of the ISGMR is a natural observable to determine the parameter $K_{\tau}$, defined as $K_{\tau}=K_{\text {sym }}-$ $6 L_{\text {sym }}-Q_{\text {sat }} L_{\text {sym }} / K_{\text {sat }}$ [44]. It represents the isoscalar curvature at the saturation density in asymmetric matter, $n_{\text {sat }}(\delta) \approx$ $n_{\text {sat }}\left(1-3 L_{\text {sat }} \delta^{2} / K_{\text {sat }}\right)$. The parameter $K_{\text {sym }}$ could, in principle, be deduced from $K_{\tau}$ if $L_{\text {sym }}$ and $Q_{\text {sat }}$ were well determined. Considering the uncertainties in these parameters, we found a very naive estimation of the error bar in $K_{\text {sym }}, \sigma \approx 600 \mathrm{MeV}$, which is certainly overestimated. Waiting for better experimental analysis in the future, the value $K_{\text {sym }}=-100 \pm 100 \mathrm{MeV}$ given in Table II is obtained from statistical analysis of various model predictions; see Sec. IIB. It is mainly related to the expected values from chiral EFT approach and is comparable with the recent analysis from unitary gas constraint [102]. Let us mention that this range for $K_{\text {sym }}$ is compatible with the one from Ref. [103] which is $-100 \pm 200 \mathrm{MeV}$. In our case, we cover the same uncertainty range considering $2 \sigma$ deviation from the central value.

We now switch to the discussion of a quantity which is usually not considered as an empirical parameter, but enters nevertheless into the important quantities which characterize nuclear matter properties. The effective mass is a powerful concept used to characterize the propagation of quasiparticles
TABLE III. Landau effective mass properties in nuclear matter at saturation density. From the estimated value of $m_{\text {sat }}^{*} / m$, we can deduce $\kappa_{s}=0.43 \pm 0.1$. See text for more details.

\begin{tabular}{lcccc}
\hline \hline Model & Ref. & $m_{\text {sat }}^{*} / m$ & $\kappa_{v}$ & $\Delta m_{\text {sat }}^{*} / m$ \\
\hline DF-Skyrme [104] & Lipparini-89 & \multicolumn{3}{c}{$0.2-0.54$} \\
DF-Skyrme [105] & Reinhard-99 & $0.8 \pm 0.1$ & $0.25 \pm 0.5$ \\
DF-Skyrme [106] & Lesinski-06 & $0.75 \pm 0.05$ & 0.6 & 0.17 \\
Opt. Pot. [107,108] & Perey-62 & $0.75 \pm 0.05$ & \\
Opt. Pot. [109] & Dover-72 & $0.75 \pm 0.03$ & \\
BHF [110] & Hassaneen-04 & $0.65 \pm 0.05$ & $0.1-0.2$ \\
DBHF [111] & Ma-04 & 0.66 & & $0.1-0.2$ \\
DBHF [112] & VanDalen-05 & 0.78 & & $0.1-0.2$ \\
Present & & $0.75 \pm 0.1$ & $0.4 \pm 0.2$ & $0.1 \pm 0.1$ \\
Estimation & & & & \\
\hline \hline
\end{tabular}

inside a strongly interacting medium, such as nuclei or nuclear matter. It reflects the nonlocality in space and time of the quasiparticle self-energy. The nonlocality in space, also called the Landau effective mass or $k$-effective mass, is related to the momentum dependence of the nuclear interaction. The Landau effective mass depends on the isoscalar and isovector densities and can be different for neutrons and protons, $m_{q}^{*}\left(n_{0}, n_{1}\right)$ where $q=n, p$. In SM, it is generally assumed that $m_{n}^{*}=m_{p}^{*}$, while in AM the neutron and proton Landau effective mass can be different. The isospin splitting of the Landau effective mass can then be expressed as

$$
\Delta m^{*}\left(n_{0}, n_{1}\right)=m_{n}^{*}\left(n_{0}, n_{1}\right)-m_{p}^{*}\left(n_{0}, n_{1}\right) .
$$

Two quantities are usually compared between various nuclear interactions: the Landau effective mass in SM at saturation $m_{\text {sat }}^{*}$ and the isospin splitting taken for $n_{0}=n_{1}=n_{\text {sat }}$ in neutron matter (NM), $\Delta m_{\mathrm{sat}}^{*}$. A summary of the determination of $m_{\mathrm{sat}}^{*}$ and $\Delta m_{\text {sat }}^{*}$ from nuclear experiments is shown in Table III.

The Landau effective mass can be extracted from the energy dependence of the optical potential which is used in phenomenological analyses of nucleon scattering data. By comparing the energy-dependent term of the real optical potential in the energy range $10-30 \mathrm{MeV}$ to the equivalent local potential from the Skyrme interaction, it was deduced that $m_{\text {sat }}^{*}$ is approximately $(0.75 \pm 0.03) m$ [109]. A similar damping of the mass was also found by Perey based on phenomenological local and nonlocal potentials giving the same phase shifts $[107,108]$. Apparently contradictory information comes from the measurement of level densities: It was indeed observed in the 1960s that the experimental level density could be reproduced only if $m_{\mathrm{sat}}^{*} \approx m$ [113]. The solution of this contradiction was found by recognizing that the mean field is not static, but it has also a dynamic component [114]: Among the modes associated with the fluctuations of the field, one finds vibrations of the nuclear surface [115-117], which is associated to an energy-dependent effective mass (or $\omega$ mass, to be distinguished from the Landau effective mass). This nonlocal-in-time property of the effective mass [118-120], however, goes beyond the scope of the present model. It is mentioned here only to illustrate the difficulty of accurately determining the effective mass from experiments. 
TABLE IV. Binding energy $E_{\text {sat }}$, the symmetry energy $E_{\text {sym }}$, saturation density $n_{\text {sat }}$, slope of the symmetry energy $L_{\text {sym }}$, isoscalar incompressibility $K_{\text {sat }}$, isovector incompressibility $K_{\text {sym }}$, isoscalar skewness $Q_{\text {sat }}$, isovector skewness $Q_{\text {sym }}$, isoscalar kurtosis $Z_{\text {sat }}$, isovector kurtosis $Z_{\text {sym }}$, the Landau effective mass at saturation $m_{\text {sat }}^{*}$, and its isospin splitting $\Delta m_{\text {sat }}^{*}$. For the relativistic approaches, the effective mass is defined to be the Landau mass derived from the equivalent Schrödinger equation; see Ref. [125] and references therein for more details.

\begin{tabular}{|c|c|c|c|c|c|c|c|c|c|c|c|c|c|c|c|}
\hline $\begin{array}{l}\text { Model } \\
\left(N_{\alpha}\right)\end{array}$ & Der. order & $\begin{array}{c}E_{\mathrm{sat}} \\
(\mathrm{MeV})\end{array}$ & $\begin{array}{c}E_{\text {sym }} \\
(\mathrm{MeV})\end{array}$ & $\begin{array}{c}n_{\mathrm{sat}} \\
\left(\mathrm{fm}^{-3}\right)\end{array}$ & $\begin{array}{c}L_{\text {sym }} \\
(\mathrm{MeV})\end{array}$ & $\begin{array}{c}K_{\text {sat }} \\
(\mathrm{MeV})\end{array}$ & $\begin{array}{c}K_{\text {sym }} \\
(\mathrm{MeV})\end{array}$ & $\begin{array}{c}Q_{\text {sat }} \\
(\mathrm{MeV})\end{array}$ & $\begin{array}{c}Q_{\text {sym }} \\
(\mathrm{MeV})\end{array}$ & $\begin{array}{c}Z_{\text {sat }} \\
(\mathrm{MeV})\end{array}$ & $\begin{array}{c}Z_{\text {sym }} \\
(\mathrm{MeV})\end{array}$ & $m_{\mathrm{sat}}^{*} / m$ & $\Delta m_{\mathrm{sat}}^{*} / m$ & $\kappa_{v}$ & $\begin{array}{c}K_{\tau} \\
(\mathrm{MeV})\end{array}$ \\
\hline & & 0 & 0 & 1 & 1 & 2 & 2 & 3 & 3 & 4 & 4 & & & & \\
\hline Skyrme & Average & -15.88 & 30.25 & 0.1595 & 47.8 & 234 & -130 & -357 & 378 & 1500 & -2219 & 0.73 & 0.08 & 0.46 & -344 \\
\hline (16) & $\sigma$ & 0.15 & 1.70 & 0.0011 & 16.8 & 10 & 66 & 22 & 110 & 169 & 617 & 0.10 & 0.24 & 0.27 & 25 \\
\hline RMF & Average & -16.24 & 35.11 & 0.1494 & 90.2 & 268 & -5 & -2 & 271 & 5058 & -3672 & 0.67 & -0.09 & 0.40 & -549 \\
\hline (11) & $\sigma$ & 0.06 & 2.63 & 0.0025 & 29.6 & 34 & 88 & 393 & 357 & 2294 & 1582 & 0.02 & 0.03 & 0.06 & 153 \\
\hline RHF & Average & -15.97 & 33.97 & 0.1540 & 90.0 & 248 & 128 & 389 & 523 & 5269 & -9956 & 0.74 & -0.03 & 0.34 & -572 \\
\hline (4) & $\sigma$ & 0.08 & 1.37 & 0.0035 & 11.1 & 12 & 51 & 350 & 237 & 838 & 4156 & 0.03 & 0.01 & 0.07 & 169 \\
\hline $\begin{array}{l}\text { lotal } \\
\text { (50) }\end{array}$ & Average & -16.03 & 33.30 & 0.1543 & 76.6 & 251 & -3 & 13 & 388 & 3925 & -5268 & 0.72 & 0.01 & 0.39 & -492 \\
\hline APR & Average & -16.0 & 33.12 & 0.16 & 50.0 & 270 & -199 & -665 & 923 & 337 & -2053 & 1.0 & 0.0 & 0.0 & -376 \\
\hline (1) & $\sigma$ & a & 0.30 & a & 1.3 & 2 & 13 & 30 & 67 & 94 & 125 & 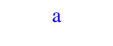 & 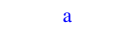 & a & 30 \\
\hline Chiral EFT & Average & -15.16 & 32.01 & 0.171 & 48.1 & 214 & -172 & -139 & -164 & 1306 & -2317 & & & & -428 \\
\hline Drischler 2016 & $\sigma_{\text {tot }}$ & 1.24 & 2.09 & 0.016 & 3.6 & 22 & 40 & 104 & 234 & 214 & 379 & & & & 63 \\
\hline \multirow[t]{2}{*}{ (7) } & Min & -16.92 & 28.53 & 0.140 & 43.9 & 182 & -224 & -310 & -640 & 901 & -2961 & & & & -534 \\
\hline & $\operatorname{Max}$ & -13.23 & 34.57 & 0.190 & 53.5 & 242 & -108 & 24 & 96 & 1537 & -1750 & & & & -334 \\
\hline
\end{tabular}

${ }^{\mathrm{a}}$ This parameter is fixed.

The Landau effective mass in Skyrme models can be expressed as [106]

$$
\frac{m}{m_{q}^{*}}=1+\kappa_{s}+\tau_{3}\left(\kappa_{s}-\kappa_{v}\right) \delta
$$

where $\kappa_{s}=m / m_{\text {sat }}^{*}-1$ in symmetric matter and $\kappa_{v}$ is the enhancement factor entering the Thomas-Reiche-Khun sum rule in the case of the isovector giant dipole resonance E1 (IVGDR) [104]. There is a direct relation between $\kappa_{s}$ and the isoscalar giant quadrupolar resonance (ISGQR) [84,105], while the value of $\kappa_{v}$ depends to a large extent on the energy region of the resonance energy [104,105].

So far, no experimental data from finite nuclei have allowed a determination of the effective mass splitting. Microscopic approaches such as BHF and DBHF have been employed and predict in a nonambiguous way that $m_{n}^{*}>m_{p}^{*}$ in neutron-rich matter [110-112]. The sign of $\Delta m^{*}$ is solidly positive, but its amplitude is not yet clearly determined and is believed to be around 0.1 to $0.2 \mathrm{~m}$. The neutron and proton Landau effective masses calculated within the Brueckner diagrammatic approach [110,112,121-124] are reported in Table III; see lines BHF (Brueckner-Hartree-Fock) and DBHF (Dirac-BruecknerHartree-Fock).

For small values of the isospin splitting $\Delta m_{\text {sat }}^{*} / m \ll 1$, the following relation is approximately satisfied:

$$
\kappa_{v} \approx \kappa_{s}-\frac{1}{2} \frac{\Delta m_{\mathrm{sat}}^{*}}{m}\left(1+\kappa_{s}\right)^{2} .
$$

To summarize, the present estimation for these parameters can be expressed as $m_{\text {sat }}^{*} / m=0.75 \pm 0.1, \kappa_{v}=0.4 \pm 0.2$, and $\Delta m_{\mathrm{sat}}^{*} / m=0.1 \pm 0.1$.

\section{B. Generic determination of the empirical parameters}

Besides the constraints determined from direct analysis of experimental data, we performed a complementary analysis of the predictions for the empirical parameters determined from various relativistic and nonrelativistic functionals. We have investigated several types of relativistic and nonrelativistic phenomenological models, namely 35 Skyrme-type functionals, 11 models based on RMF effective Lagrangians, four RHF effective Lagrangians, as well as two more ab initio approaches, APR and chiral EFT. For simplicity, APR and chiral EFT EOS are grouped together since they are both based on the $N N$ interaction in vacuum, at variance with the so-called phenomenological approaches. The interactions on which they are based are, however, very different in nature, but this goes beyond the present analysis. Concerning the phenomenological models, we report in the appendix the isoscalar and isovector empirical parameters up to the fourth order, the Landau effective mass at saturation $m_{\text {sat }}^{*}$, and its isospin splitting $\Delta m_{\text {sat }}^{*}$; see Tables X, XI, and XII. Details for $a b$ initio chiral EFT approach are discussed in Sec. IV B.

In Table IV, we present a summary of the detailed results shown in the appendix and Sec. IV B: The average values for each type of model (Skyrme, RMF, and RHF) are calculated as well as the standard deviation $\sigma$ for each type of model, 
defined as $\sigma^{2}=\sum_{i}\left[x_{i}^{2}-\langle x\rangle\right] / N_{\text {models }}$, where $x$ stands for an empirical parameter and $N_{\text {models }}$ is the number of parameter sets for each type of modeling $\left(N_{\text {models }}=N_{\alpha}\right)$ given in Table IV. Note that for the Skyrme-type models, we present two different averages, over 16 and 35 models, respectively. Our sampling of Skyrme forces is more limited than in other analysis; see, for instance, Ref. [18] and references therein. The 35 Skyrme forces that we have considered here are among the mostly used forces. In addition, the reduced sampling of 16 Skyrme functionals contains the forces which are usually employed for finite nuclei. Since some groups have produced many different forces, but with rather similar constraints, we have decided to consider only a few of these forces in our sample. In doing so, we limit as much as possible the bias which may come from the details of the fit and give almost equal weights to different groups, thus increasing the meaning of the calculated average and standard deviations. The test of the stability of our statistical analysis is performed by comparing the small sample to the wider one. The central values are shown to be rather independent of the sampling, while the standard deviation $\sigma$ increases with the number of models.

Considering the group of phenomenological approaches, the last lines of this group in Table IV provide the average values, the standard deviations $\sigma_{\text {tot }}$, and the minimal and maximal values found for all empirical parameters. The average and the standard deviation could be influenced by the number of models belonging to each type of model. In order to reduce this influence, the average and standard deviation are weighted differently for the three different type of models. The mean value is defined as $\sum_{\alpha} 1 / 3 \sum_{i} x_{i} / N_{\alpha}$, where $\alpha$ runs over the three types of models (Skyrme, RMF, and RHF) and $i$ over the models themselves. This is strictly identical to take the arithmetic mean of the three first average values given in the first lines of Table IV. In a similar way, the standard deviation is defined as $\sigma_{\text {tot }}^{2}=\sum_{\alpha} 1 / 3 \sum_{i}\left[x_{i}^{2}-\langle x\rangle\right] / N_{\alpha}$, where $N_{\alpha}$ is given in the first column of Table IV and the mean value $\langle x\rangle$ is the one of the final average considering the 50 models. By comparing different types of phenomenological approaches, we expect that the final central values and central deviations that we obtained are weakly impacted by the choice of the samples, provided only models used in finite nuclei are considered.

For the group of $a b$ initio approaches, the same statistical quantities are generated from the seven chiral EFT results. For APR, we have fitted Eq. (5) to the APR symmetric and neutron matter EOS and we provide in Table IV the best fit and its associated error bar.

It is clear from Table IV that all the empirical parameters are model dependent, even the lowest order ones: For instance, the average saturation energies $E_{\text {sat }}$ and densities $n_{\text {sat }}$ are different among Skyrme, RMF, and RHF type of models, and the differences among these average values are larger than the standard deviations. The same remark applies also for the $a b$ initio approaches. This indicates a model dependence for these quantities. It is particularly interesting to remark on the big deviations for the empirical parameters $K_{\text {sym }}$ and $Q_{\text {sat }}$ among the Skyrme, RMF, and RHF models. These two quantities are predicted to be negative for Skyrme interactions, compatible with zero for RMF, and positive (almost equal in absolute value to the Skyrme models) for the RHF approaches. This is an indication that these values are weakly constrained by their fitting protocol, which is mostly based on nuclear masses and charge radii. The higher order empirical parameters $\left(Q_{\text {sym }}\right.$, $Z_{\text {sat }}, Z_{\text {sym }}$ ) are quite unknown, as shown by the fact that their standard deviations are comparable to their average values. Finally, there is also a quite large model dependence for the effective mass $m_{\text {sat }}^{*}$ and the isospin splitting $\Delta m_{\text {sat }}^{*}$.

It is interesting to note from Table IV that the values obtained for the empirical parameters $E_{\text {sat }}, E_{\text {sym }}, n_{\text {sat }}, K_{\text {sat }}$, $L_{\text {sym }}$, and $K_{\tau}$ are rather close to the ones extracted from an analysis of experimental data, as discussed in Sec. II A. This is also the case for the so-called ab initio approaches, except for $n_{\text {sat }}$ which is slightly too high for the chiral EFT case. This is, however, a general issue shared by ab initio approaches [9]. Except for the value of $Q_{\text {sym }}$, the average Skyrme and chiral EFT predictions match in a satisfactory way. The value for $Q_{\text {sat }}$ is systematically lower for Skyrme and chiral EFT than for the relativistic phenomenological approaches. This makes the nonrelativistic EOS generally softer than the relativistic ones. This good match between the low-order empirical parameters deduced from the statistical average and shown in Table IV with the experimental data discussed in Sec. II A indicates that the estimated values provided by Table IV are reasonably well constrained. It appears therefore reasonable to take the values of Table IV also for the empirical parameters for which there are no experimental data.

Some $a b$ initio calculations provide only the neutron matter (NM) EOS, since it does not present the extra complication of the spinodal instability at low density. The NM EOS is obtained in the metamodel by taking the value $\delta=1$ in Eq. (5). At each order, the two isoscalar and isovector coefficients become a single coefficient that we indicate in the following with index NM. Note that since the pressure of symmetric matter at saturation density is zero, $L_{\mathrm{NM}}=L_{\text {sym }}$. In Table V, we show the predictions for the NM empirical parameters of the same approaches as in Table IV plus a couple of other $a b$ initio predictions: GCR 2012 [126] and chiral EFT Tews 2013 [11]. Details on how these numbers have been obtained for GCR 2012 and chiral EFT Tews 2013 are given in Sec. IV B. It is worth noticing that the different $a b$ initio approaches give consistent estimation for $K_{\mathrm{NM}}$ between 120 and $40 \mathrm{MeV}$. Since $K_{\mathrm{NM}}=K_{\text {sat }}+K_{\text {sym }}$ and $K_{\text {sat }}=230 \pm 20 \mathrm{MeV}$, we have approximately $K_{\text {sym }} \approx-100 \pm 100 \mathrm{MeV}$ (including also the preferred values from RMF and RHF approaches). The Skyrme and $a b$ initio approaches prefer values $K_{\text {sym }} \approx$ $-200,-150$ while the relativistic approaches prefer $K_{\text {sym }} \approx$ $0,100 \mathrm{MeV}$.

Let us mention another phenomenological approach, the so-called two-loop quantum hadrodynamics, which is based on RMF with an adjunction of the two-loop exchange diagrams [127]. For fixed values of $E_{\text {sat }}, n_{\text {sat }}, K_{\text {sat }}$, and $E_{\text {sym }}$ comparable with the ones in Table IV, this approach predicts $L_{\text {sym }} \approx$ $83-85 \mathrm{MeV}$ and $K_{\text {sym }} \approx-20 \mathrm{MeV}$, which is in the range of values that we explore.

To conclude this analysis, we now discuss the total average and total standard deviation $\sigma_{\text {tot }}$ shown in Table IV. They provide a global estimation for the empirical parameters including the systematic error bar induced by the model dependence, as 
TABLE V. Neutron matter energy per nucleon $E_{\mathrm{NM}}$, slope of the symmetry energy $L_{\text {sym }}$, neutron matter incompressibility $K_{\mathrm{NM}}$, neutron matter skewness $Q_{\mathrm{NM}}$, and neutron matter kurtosis $Z_{\mathrm{NM}}$ for phenomenological and $a b$ initio approaches. See text for more details as well as Appendix IV B, deduced for GCR 2012 [126] and chiral EFT up to $\mathrm{N}^{3}$ LO [11].

\begin{tabular}{|c|c|c|c|c|c|c|}
\hline $\begin{array}{l}\text { Model } \\
\left(N_{\alpha}\right)\end{array}$ & & $\begin{array}{c}E_{\mathrm{NM}} \\
(\mathrm{MeV})\end{array}$ & $\begin{array}{c}L_{\text {sym }} \\
(\mathrm{MeV})\end{array}$ & $\begin{array}{c}K_{\mathrm{NM}} \\
(\mathrm{MeV})\end{array}$ & $\begin{array}{c}Q_{\mathrm{NM}} \\
(\mathrm{MeV})\end{array}$ & $\begin{array}{c}Z_{\mathrm{NM}} \\
(\mathrm{MeV})\end{array}$ \\
\hline \multicolumn{7}{|c|}{ Phenomenological approaches } \\
\hline Skyrme & Average & 14.95 & 49.6 & 106 & 21 & -727 \\
\hline (35) & $\sigma$ & 1.72 & 21.6 & 116 & 276 & 1580 \\
\hline $\mathrm{RMF}$ & Average & 18.86 & 90.2 & 263 & 269 & 1386 \\
\hline (11) & $\sigma$ & 2.69 & 29.6 & 121 & 750 & 3876 \\
\hline RHF & Average & 17.99 & 90.0 & 376 & 912 & -4686 \\
\hline (4) & $\sigma$ & 1.46 & 11.1 & 63 & 587 & 4994 \\
\hline \multicolumn{7}{|c|}{ Ab initio approaches } \\
\hline APR & Average & 17.27 & 50.0 & 71 & 258 & -1716 \\
\hline (1) & $\sigma$ & 0.30 & 1.3 & 15 & 97 & 219 \\
\hline GCR 2012 & Average & 16.76 & 45.8 & 77 & 80 & -131 \\
\hline (7) & $\sigma$ & 1.39 & 9.7 & 43 & 29 & 15 \\
\hline Chiral EFT & Average & 16.39 & 56.4 & 119 & & \\
\hline Tews 2013 & $\sigma$ & 2.97 & 11.0 & 101 & & \\
\hline Chiral EFT & Average & 16.93 & 48.3 & 41 & -314 & -991 \\
\hline Drischler 2016 (7) & $\sigma$ & 0.92 & 3.5 & 33 & 226 & 349 \\
\hline
\end{tabular}

previously discussed. From these global results, it is possible to separate the empirical quantities into four groups:

(1) The parameters which are known within a few percent: $E_{\text {sat }}$ and $n_{\text {sat }}$.

(2) The parameters which are known within about $10 \%$ : $E_{\text {sym }}, K_{\text {sat }}$, and $m_{\text {sat }}^{*}$.

(3) The parameters which are known within about 50\%: $L_{\text {sym }}$.

(4) The parameters which are almost unknown: $Q_{\text {sat }}, Z_{\text {sat }}$, $K_{\text {sym }}, Q_{\text {sym }}, Z_{\text {sym }}$, and $\Delta m_{\text {sat }}^{*}$.

We may hope that the empirical parameters in the three first groups will be better constrained from nuclear physics experiments in the future, considering in addition to the masses and charge radii constraints the ones provided by the neutron skin radii, the collective modes in neutron-rich nuclei, and possibly large deformations in the ground state. It is, however, hard to imagine that the parameters from the last group will ever be constrained from the properties of finite nuclei around saturation density. To be better determined, they require the knowledge of the properties of systems at densities and asymmetries different from those of finite nuclei. It could be expected that the Heavy Ion Collision (HIC) will provide some constraints, as well as the observed properties of compact stars. This will be further discussed in the following sections.

\section{A METAMODELING FOR THE NUCLEAR EQUATION OF STATE}

In this section, we investigate to which extent a series expansion of the same kind as the one given by Eqs. (2)-(4) can generate a realistic equation of state (EOS). There are two questions to answer, which are as follows: (i) Are the density and isospin dependence rich enough? (ii) What is the convergence in density and isospin parameter of such series expansions?

A purely polynomial density expansion as in Eqs. (2)-(4) is too simple to provide realistic results because it does not catch the natural density and isospin dependence of the kinetic term [46]. For this reason, we will separate the kinetic term from the potential one (2)-(4).

To fully cover the parameter space of both relativistic and nonrelativistic models, the best treatment of the kinetic term would be an expansion in powers of the Fermi momentum $k_{F}[128,129]$. This would, however, introduce a high number of extra poorly constrained parameters. We have therefore chosen to limit ourselves to a nonrelativistic treatment for this paper, such that the kinetic term can be exactly handled and the expansion only concerns the Landau effective mass. It is important to remark that, even if the kinetic energy density is treated nonrelativistically, the functional is still flexible enough to satisfactorily reproduce also the density dependence of relativistic models. This point will be demonstrated in Sec. IV A.

Let us also mention that an expansion of the energy per nucleon in terms of the Fermi momentum $k_{F}$ is also possible [38]. In our present study, we aim at keeping a simple relation between the empirical parameters and the parameters of the model. This determines our choice for an expansion in powers of the density.

The metamodel on which the EOS is based on has therefore four requirements:

(1) The nuclear potential is quadratic in the isospin asymmetry parameter $\delta$.

(2) The EOS is analytic in the parameter $x$, and possible phase transitions are not accounted for.

(3) The energy per nucleon satisfies the following limit: $\lim _{n_{0} \rightarrow 0} e\left(n_{0}, n_{1}\right)=0$.

From the functional form of the energy per nucleon $e\left(n_{0}, n_{1}\right)$, it is possible to calculate analytically its first- and second-order derivatives, which are related to the nucleon pressure and to the nucleon sound velocity as

$$
\begin{aligned}
P_{n}\left(n_{0}, n_{1}\right) & =n_{0}^{2} \frac{\partial e}{\partial n_{0}}, \\
\left(\frac{v_{s, n}}{c}\right)^{2} & =\frac{K_{\text {is }}\left(n_{0}, n_{1}\right)}{9\left[m c^{2}+e+\frac{P\left(n_{0}, n_{1}\right)}{n_{0}}\right]},
\end{aligned}
$$

where the isoscalar compressibility $K_{\text {is }}\left(n_{0}, n_{1}\right)$ is defined as

$$
K_{\text {is }}\left(n_{0}, n_{1}\right)=9 n_{0}^{2} \frac{\partial^{2} e}{\partial n_{0}^{2}}+18 \frac{P\left(n_{0}, n_{1}\right)}{n_{0}} .
$$

Note that $K_{\text {is }}\left(n_{\text {sat }}, 0\right)=K_{\text {sat }}$

The symmetry energy is defined as

$$
S\left(n_{0}\right)=\left.\frac{1}{2} \frac{\partial^{2} e\left(n_{0}, n_{1}\right)}{\partial \delta^{2}}\right|_{n_{1}=0} .
$$


In the following, we first express the kinetic energy contribution to the total energy, and then we explore various approximations for the potential energy.

\section{A. The kinetic energy term}

For a nonrelativistic free Fermi gas (FG), the kinetic energy per particle is simply given by

$$
t^{\mathrm{FG}}\left(n_{0}, n_{1}\right)=\frac{t_{\mathrm{sat}}^{\mathrm{FG}}}{2}\left(\frac{n_{0}}{n_{\mathrm{sat}}}\right)^{2 / 3} f_{1}(\delta),
$$

where $t_{\text {sat }}^{\mathrm{FG}}=3 \hbar^{2} /(10 m)\left(3 \pi^{2} / 2\right)^{2 / 3} n_{\text {sat }}^{2 / 3}$ is the kinetic energy per nucleons in SM and at saturation, $m$ is the nucleonic mass taken identical for neutrons and protons $\left(m=\left(m_{n}+m_{p}\right) / 2=\right.$ $938.919 \mathrm{MeV} / \mathrm{c}^{2}$ ), giving $t_{\mathrm{sat}}^{\mathrm{FG}} \approx 22.1 \mathrm{MeV}$, and the function $f_{1}$ is defined as

$$
f_{1}(\delta)=(1+\delta)^{5 / 3}+(1-\delta)^{5 / 3} .
$$

The momentum dependence of the nuclear interaction gives rise to the concept of effective mass: An average effect of the in-medium nuclear interaction is to modify the inertial mass of the nucleons. The Landau effective mass can be parameterized in the following way $(\tau=n$ or $p$ ),

$$
\frac{m}{m_{\tau}^{*}\left(n_{0}, n_{1}\right)}=1+\left(\kappa_{\mathrm{sat}}+\tau_{3} \kappa_{\mathrm{sym}} \delta\right) \frac{n_{0}}{n_{\mathrm{sat}}},
$$

where $\tau_{3}=1$ for neutrons and -1 for protons, and where the parameters $\kappa_{\text {sat }}$ and $\kappa_{\text {sym }}$ are functions of $m_{\text {sat }}^{*}$ and $\Delta m_{\text {sat }}^{*}$ previously discussed; see Sec. II A. We have for both $\kappa_{\text {sat } / \text { sym }}$ the following expressions taken at $n_{0}=n_{\text {sat }}$ :

$$
\begin{aligned}
\kappa_{\mathrm{sat}} & =\frac{m}{m_{\mathrm{sat}}^{*}}-1=\kappa_{s} \text { in } \operatorname{SM}(\delta=0), \\
\kappa_{\mathrm{sym}} & =\frac{1}{2}\left[\frac{m}{m_{n}^{*}}-\frac{m}{m_{p}^{*}}\right] \text { in } \mathrm{NM}(\delta=1) .
\end{aligned}
$$

Introducing the parameters $\kappa_{s}$ and $\kappa_{v}$, we have $\kappa_{\text {sat }}=\kappa_{s}$ and $\kappa_{\text {sym }}=\kappa_{s}-\kappa_{v}$ [106]. The functional form (15) for the inmedium effective mass is the simplest form of a density series expansion. Truncating the expansion at first order as in (15) allows us to recover the expression used in standard Skyrme functionals. For simplicity, we do not generalize Eq. (15) with a more complete polynomial in this work. Anticipating results presented in Sec. V, it will be shown that the impact of the effective mass on the equation of state is very weak (at zero temperature), justifying our present approximation.

Considering the functional form (15) for the nucleonic effective mass, the new expression for the kinetic energy in nuclear matter reads

$$
\begin{aligned}
t^{\mathrm{FG}}\left(n_{0}, n_{1}\right)= & \frac{t_{\mathrm{sat}}^{\mathrm{FG}}}{2}\left(\frac{n_{0}}{n_{\mathrm{sat}}}\right)^{2 / 3}\left[\left(1+\kappa_{\mathrm{sat}} \frac{n_{0}}{n_{\mathrm{sat}}}\right) f_{1}(\delta)\right. \\
& \left.+\kappa_{\mathrm{sym}} \frac{n_{0}}{n_{\mathrm{sat}}} f_{2}(\delta)\right],
\end{aligned}
$$

where the new function $f_{2}$ is defined as

$$
f_{2}(\delta)=\delta\left((1+\delta)^{5 / 3}-(1-\delta)^{5 / 3}\right) .
$$

In the following, the kinetic energy contribution to the density functional will be given by Eq. (17), which is the simplest way to consider the contribution of the momentum dependence of the nuclear interaction.

This expression gives the exact kinetic energy density only if we want to reproduce models with nonrelativistic kinematics. In the case of relativistic EOS models, it would be more natural to employ a relativistic formulation for the kinetic energy density and use Dirac masses instead of Landau masses as nonlocal empirical parameters. This will certainly be necessary if we want to adress specific observables which are especially sensitive to the kinetic energy term. We also expect that isolating a relativistic kinetic energy density term from the polynomial expansion will improve the convergence of the series when reproducing relativistic models, and such an extension toward a relativistic metamodeling is planned for the future.

Concerning the energy per particle and the pressure of homogeneous matter, which are our main concerns here, we will see in Sec. IV that RMF and RHF models are also satisfactorily reproduced by our metamodeling, even if the degree of reproduction is less accurate than for nonrelativistic ones.

We now discuss the functional form for the potential energy.

\section{B. Metamodeling ELFa: The simplest approach}

Once the kinetic energy density is sorted out via Eq. (17), the energy per nucleon can be written as

$$
e_{E L F a}^{N}\left(n_{0}, n_{1}\right)=t^{\mathrm{FG} *}\left(n_{0}, n_{1}\right)+v_{E L F a}^{N}\left(n_{0}, n_{1}\right),
$$

where the potential energy is expressed as a series expansion in the parameter $x$,

$$
v_{E L F a}^{N}\left(n_{0}, n_{1}\right)=\sum_{\alpha \geqslant 0}^{N} \frac{1}{\alpha !} v_{\alpha}(\delta) x^{\alpha} .
$$

Since Eq. (19) provides an empirical local density functional (ELF), this metamodeling is called ELFa.

Supposing a quadratic approximation for the potential energy, as suggested by microscopic Bruckner calculations [53], we have

$$
v_{\alpha}(\delta)=v_{\alpha}^{\text {is }}+v_{\alpha}^{\text {iv }} \delta^{2} .
$$

Simple relations can be obtained between the model parameters $v_{\alpha}^{\text {is }}$ and $v_{\alpha}^{\text {iv }}$ and the empirical parameters. We have for the isoscalar parameters

$$
\begin{aligned}
& v_{\alpha=0}^{\text {is }}=E_{\text {sat }}-t_{\text {sat }}^{\mathrm{FG}}\left(1+\kappa_{\text {sat }}\right), \\
& v_{\alpha=1}^{\text {is }}=-t_{\text {sat }}^{\mathrm{FG}}\left(2+5 \kappa_{\text {sat }}\right), \\
& v_{\alpha=2}^{\text {is }}=K_{\text {sat }}-2 t_{\text {sat }}^{\mathrm{FG}}\left(-1+5 \kappa_{\text {sat }}\right), \\
& v_{\alpha=3}^{\text {is }}=Q_{\text {sat }}-2 t_{\text {sat }}^{\mathrm{FG}}\left(4-5 \kappa_{\text {sat }}\right), \\
& v_{\alpha=4}^{\text {is }}=Z_{\text {sat }}-8 t_{\text {sat }}^{\mathrm{FG}}\left(-7+5 \kappa_{\text {sat }}\right),
\end{aligned}
$$

and the isovector parameters

$$
\begin{aligned}
& v_{\alpha=0}^{\mathrm{iv}}=E_{\mathrm{sym}}-\frac{5}{9} t_{\mathrm{sat}}^{\mathrm{FG}}\left[1+\left(\kappa_{\mathrm{sat}}+3 \kappa_{\mathrm{sym}}\right)\right], \\
& v_{\alpha=1}^{\mathrm{iv}}=L_{\mathrm{sym}}-\frac{5}{9} t_{\mathrm{sat}}^{\mathrm{FG}}\left[2+5\left(\kappa_{\mathrm{sat}}+3 \kappa_{\mathrm{sym}}\right)\right],
\end{aligned}
$$




$$
\begin{aligned}
& v_{\alpha=2}^{\mathrm{iv}}=K_{\mathrm{sym}}-\frac{10}{9} t_{\mathrm{sat}}^{\mathrm{FG}}\left[-1+5\left(\kappa_{\mathrm{sat}}+3 \kappa_{\mathrm{sym}}\right)\right], \\
& v_{\alpha=3}^{\mathrm{iv}}=Q_{\mathrm{sym}}-\frac{10}{9} t_{\mathrm{sat}}^{\mathrm{FG}}\left[4-5\left(\kappa_{\mathrm{sat}}+3 \kappa_{\mathrm{sym}}\right)\right], \\
& v_{\alpha=4}^{\mathrm{iv}}=Z_{\mathrm{sym}}-\frac{40}{9} t_{\mathrm{sat}}^{\mathrm{FG}}\left[-7+5\left(\kappa_{\mathrm{sat}}+3 \kappa_{\mathrm{sym}}\right)\right] .
\end{aligned}
$$

The simple one-to-one correspondence between the model parameters and the empirical parameter is coming from the expansion of the potential energy in the parameter $x$. It is therefore related to the fact that only integer powers of the density are considered. Another interesting aspect of the series expansion (20) is that we have a clear control on the derivative up to which we think we can determine to potential contribution. For derivative of higher order than the limit $N$ there is no contribution from the potential energy and only the kinetic energy contributes.

In the metamodel ELFa, the parameter $N$ (integer) defines the highest power in $x$. It can vary from 0 to $\infty$. As $N$ is larger, the series expansion becomes better. In order to illustrate the contribution of the different orders in $N$, we show in Fig. 2 the energy per nucleon in symmetric matter as function of $N$ going from 0 to 4 at maximum, and for a range of densities going from $n_{0}=0$ to $0.6 \mathrm{fm}^{-3}$. The values for the empirical parameters are taken from the Skyrme interaction SLy5, and for comparison the energy per nucleon given by SLy5 is also shown in Fig. 2. We first remark that the different metamodels ELFa pass through the saturation point, but only the models with $N \geqslant 2$ reproduce the saturation properties with a minimum value for the energy per nucleon at the saturation point. As the density departs from the saturation density, the models with largest value in $N$ get closer to the reference model given by SLy5 (crosses in Fig. 2).

The inset in Fig. 2 shows in more detail the low-density behavior of the energy per nucleon given by the model ELFa for various $N$. The main default of the model is that the potential energy is not zero at $n_{0}=0$. Since the model is a series expansion around the saturation density $n_{\text {sat }}$, it is indeed not given that the energy per nucleon goes to zero when the density goes to zero. In the following, we propose two modifications of ELFa, namely ELFb and ELFc, for curing this issue at zero density.

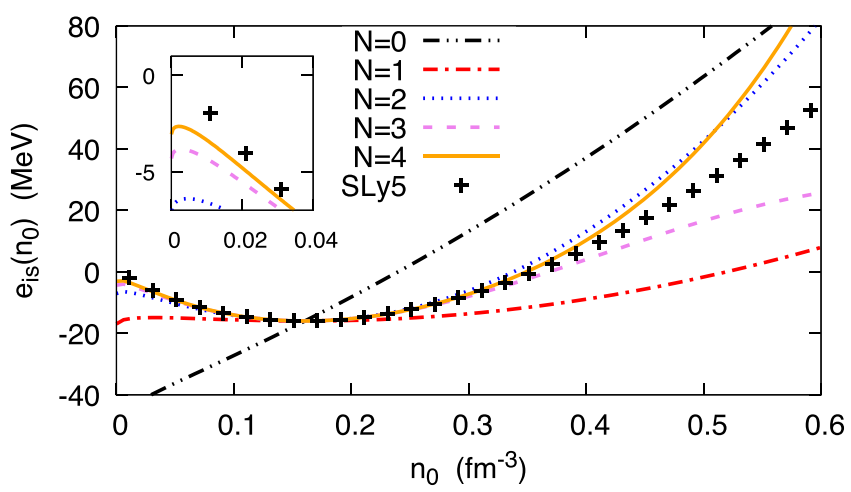

FIG. 2. Comparison of the energy per nucleon in symmetric matter between Skyrme SLy5 and ELFa metamodeling, where the empirical parameters of Skyrme SLy5 has been used as a function of the order $N$ (lines with different colors). The crosses show the reference value given by SLy5.

\section{Metamodeling ELFb: A correction at zero density}

In this section, we still express the energy per nucleon in the following way:

$$
e_{E L F b}^{N}\left(n_{0}, n_{1}\right)=t^{\mathrm{FG} *}\left(n_{0}, n_{1}\right)+v_{E L F b}^{N}\left(n_{0}, n_{1}\right) .
$$

A way to ensure that the zero-density limit is verified is to change the Taylor expansion around $n_{\text {sat }}$ from metamodel ELFa to a polynomial expansion in terms of the density $n_{0}$, as

$$
v_{E L F b}^{N}\left(n_{0}, n_{1}\right)=\sum_{\alpha \geqslant 1}^{N}\left(p_{\alpha}^{\text {is }}+p_{\alpha}^{\text {iv }} \delta^{2}\right) n_{0}^{\alpha} .
$$

The expression (33) has been used in various functionals; see, for instance, Refs. [130,131]. It can, however, be shown that this expansion is strictly equivalent to an expansion around the saturation density,

$$
v_{E L F b}^{N}\left(n_{0}, n_{1}\right)=\sum_{\alpha \geqslant 0}^{N} \frac{1}{\alpha !}\left(v_{\alpha}^{\text {is }}+v_{\alpha}^{\text {iv }} \delta^{2}\right) x^{\alpha},
$$

where the parameters $v_{N}^{\text {is }}$ and $v_{N}^{\text {iv }}$ are fixed by the zero-density limit to be

$$
v_{N}^{\text {is } / \text { iv }}=-\sum_{\alpha \geqslant 0}^{N-1} \frac{N !}{\alpha !} v_{\alpha}^{\text {is/iv }}(-3)^{N-\alpha}
$$

while the model parameters $v_{\alpha}^{\text {is/iv }}$ for $\alpha<N$ are still related to the empirical parameters according to Eqs. (22)-(31).

Combining Eqs. (34) and (35) together, the potential energy can be rewritten as

$$
v_{E L F b}^{N}\left(n_{0}, n_{1}\right)=\sum_{\alpha \geqslant 0}^{N-1} \frac{1}{\alpha !}\left(v_{\alpha}^{\text {is }}+v_{\alpha}^{\text {iv }} \delta^{2}\right) x^{\alpha} u_{E L F b, \alpha}^{N}(x),
$$

where $u_{E L F b, \alpha}^{N}(x)=1-(-3 x)^{N-\alpha}$.

The energy per nucleon deduced from the metamodeling $\mathrm{ELFb}$ is shown in Fig. 3 and can be compared to the previous Fig. 2 for the metamodel ELFa. The zero-density limit is now well satisfied, as shown in the inset figure; however, the convergence ordering with $N$ beyond saturation density is missing with metamodeling ELFb: There is no improvement of the convergence by increasing $N$. This breaking of the convergence ordering observed with metamodeling $\mathrm{ELFb}$ is

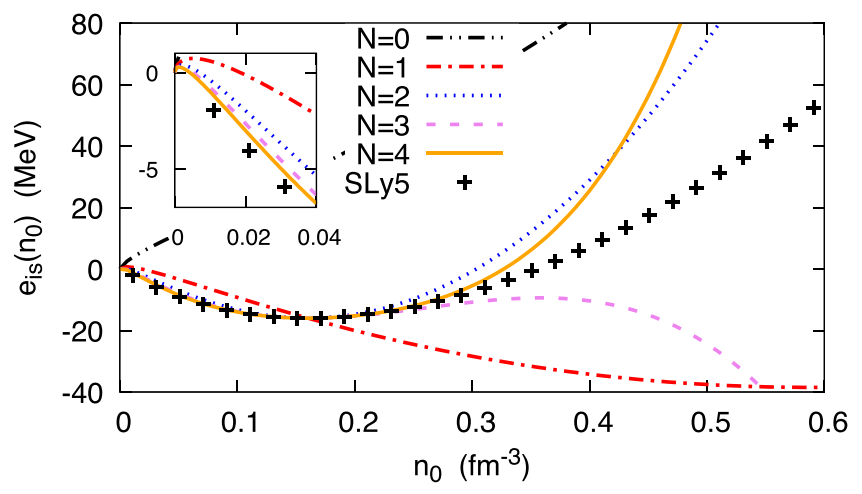

FIG. 3. Same as Fig. 2 for ELFb metamodeling. 
not very surprising since the parameters $v_{N}^{\text {is }}$ and $v_{N}^{\text {iv }}$, which govern the high-density behavior of the energy per nucleon, are now uniquely determined by the zero-density limit. Figure 3 illustrates that the density dependence of the energy per nucleon below and above saturation density is not symmetric, and a condition improving the low-density behavior of the energy per nucleon can strongly deteriorate the properties of the EOS above saturation density.

\section{Metamodeling ELFc: An improved correction at zero density}

Since metamodeling ELFb has shown that the behavior below and above saturation density of the energy per nucleon are essentially disconnected, we investigate with metamodeling ELFc an alternative approach which breaks the symmetry around saturation density. In metamodeling ELFc, a densitydependent term is added at low density in order to satisfy the zero-density limit, and this term drops to zero as the density increases. Since the small correction acts only at very low density, the properties of ELFa around and above saturation density are entirely conserved. The small term is determined such that (i) it decreases quickly with the density, (ii) it does not modify the relation between the model parameters and the empirical quantities (22)-(31), and (iii) it is fixed to annihilate the finite value given by an expansion (20). Considering these requirements, we consider the following expression for the potential energy:

$$
\begin{aligned}
v_{E L F c}^{N}\left(n_{0}, n_{1}\right)= & \sum_{\alpha \geqslant 0}^{N} \frac{1}{\alpha !}\left(v_{\alpha}^{\text {is }}+v_{\alpha}^{\text {iv }} \delta^{2}\right) x^{\alpha}-\left(a_{N}^{\text {is }}+a_{N}^{\text {iv }} \delta^{2}\right) \\
& \times x^{N+1} \exp \left(-b \frac{n_{0}}{n_{\text {sat }}}\right) .
\end{aligned}
$$

The numerical values for the coefficients $a_{N}^{\text {is }}$ and $a_{N}^{\text {iv }}$ are fixed such that the potential energy in Eq. (37) is zero at zero density. These parameters are functions of the order $N$ of the expansion and are defined as

$$
\begin{aligned}
& a_{N}^{\mathrm{is}}=-\sum_{\alpha \geqslant 0}^{N} \frac{1}{\alpha !} v_{\alpha}^{\mathrm{is}}(-3)^{N+1-\alpha}, \\
& a_{N}^{\mathrm{iv}}=-\sum_{\alpha \geqslant 0}^{N} \frac{1}{\alpha !} v_{\alpha}^{\mathrm{iv}}(-3)^{N+1-\alpha} .
\end{aligned}
$$

In Fig. 4, the $N$ dependence of the coefficients $a_{N}^{\text {is }}$ and $a_{N}^{\text {iv }}$ is shown for a set of relativistic (bottom panels) and nonrelativistic (top panels) interactions. It is interesting to remark that as $N$ increases, the absolute value of $a_{N}^{\text {is }}$ and $a_{N}^{\text {iv }}$ decreases to a small number. Therefore, as $N$ is larger, the correction at low density becomes smaller.

The condition at zero density determining the value of the coefficients $a_{N}^{\text {is }}$ and $a_{N}^{\text {iv }}$ does not fix the parameter $b$, which remains free to determine. In the following, it is fixed such that the correction quickly vanishes at small but finite density. Imposing that the exponential function in Eq. (37) is $1 / 2$ at $n_{0}=0.1 n_{\text {sat }}$, we obtain $b=10 \ln 2 \approx 6.93$. Note that the results shown in this paper are not impacted by the choice for the constant $b$ since the correction term plays a role
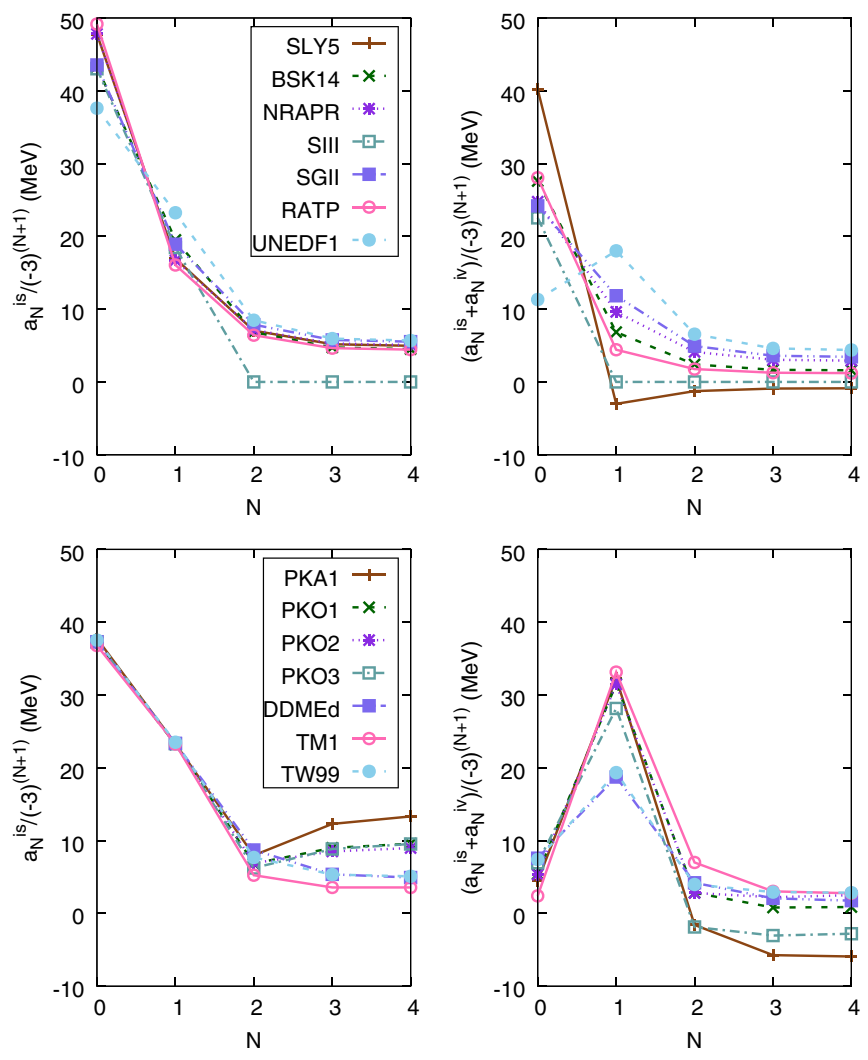

FIG. 4. Coefficients $a_{N}^{\text {is }} /(-3)^{N+1}$ (left) and $a_{N}^{\text {iv }} /(-3)^{N+1}$ (right) as a function of the order $N$ for various relativistic (bottom panels) and nonrelativistic (top panels) nuclear interactions.

only at low density and we focus our study above saturation density.

Finally, the following compact form for the potential energy can be obtained,

$$
v_{E L F c}^{N}\left(n_{0}, n_{1}\right)=\sum_{\alpha \geqslant 0}^{N} \frac{1}{\alpha !}\left(v_{\alpha}^{\text {is }}+v_{\alpha}^{\text {iv }} \delta^{2}\right) x^{\alpha} u_{E L F c, \alpha}^{N}(x),
$$

where $u_{E L F c, \alpha}^{N}(x)=1-(-3 x)^{N+1-\alpha} \exp \left(-b n_{0} / n_{\text {sat }}\right)$, and the energy per particle is defined as

$$
e_{E L F c}^{N}\left(n_{0}, n_{1}\right)=t^{\mathrm{FG} *}\left(n_{0}, n_{1}\right)+v_{E L F c}^{N}\left(n_{0}, n_{1}\right) .
$$

The energy per nucleon provided by metamodeling ELFc is shown in Fig. 5 for various orders $N$. We can see that the convergence at high density is the same as in the metamodeling ELFa (see Fig. 2), while the low-density behavior is now correct. In the following, we now study in more detail the convergence of the energy per nucleon above saturation density and we propose a way to fix the high-order parameters such as to reproduce existing nuclear interaction predictions.

\section{E. Metamodeling ELFd: A faster convergence at high density}

The high-density convergence of the metamodeling ELFc with $N$ is not entirely satisfying, as illustrated in Fig. 5. First, it shall be remarked that the empirical parameters increase (in 


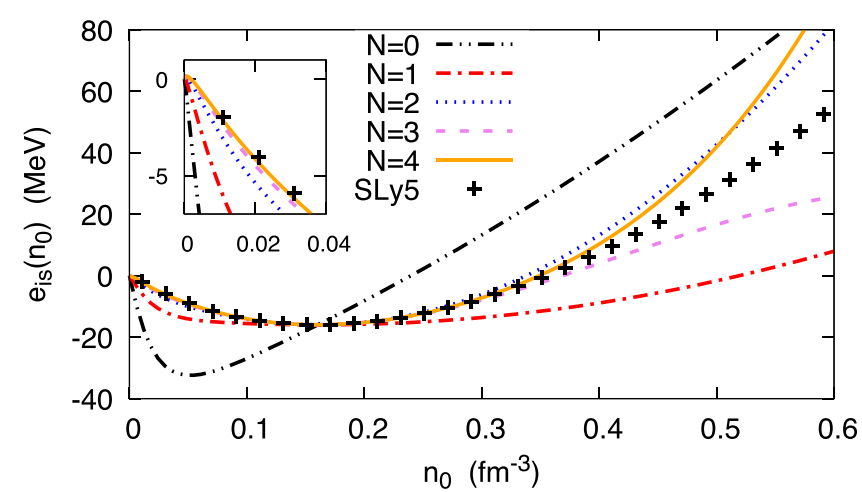

FIG. 5. Same as Fig. 2 for ELFc metamodeling.

absolute value) as the order $\alpha$ increases; see Table IV. Second, the sign of the empirical parameters alternates at each order for $\alpha \geqslant 3$. These two properties of the empirical parameters make the convergence lengthy, meaning that an important number of high-order derivatives are needed to determine the high-density behavior. Moreover, the impact of the high-order empirical parameters around saturation is extremely small. This means that, as we have already discussed, nuclear structure data and $a b$ initio calculations will hardly be able to provide reliable values for these parameters. In this respect, the situation might look hopeless. However, we can observe that the density behavior of a purely nucleonic equation of state above saturation is rather smooth and does not show any complicated structure in all existing models; see, for instance, model SLy5 represented in Fig. 5. This observation implies that the globality of the high-order parameters would be pretty much under control if we would fit these high-order parameters directly to the EOS. Equivalently, we could also impose the value of the EOS at a single high-density point, in addition to the empirical information around saturation. We have checked that there is no major differences between the fit of the high-density behavior of the EOS and the choice of a single high-density point, except that the latter allows for analytical expressions.

To give an illustration of this statement, let us suppose that the energy per nucleon and the pressure are known at $n_{0}=4 n_{\text {sat }}=n_{\text {hd }}$, where $h d$ means high density. The reference density $n_{\text {hd }}$ is quite arbitrary in this illustration; it could also have been chosen at a lower density. The advantage of the present choice is in the simplification of the equations.

Considering $N=3$, the parameters $Q_{\text {sat } / \text { sym }}$ can then be fixed such that $e_{E L F d}^{N}\left(n_{0}=n_{\mathrm{hd}}, n_{1}=0\right)=e_{\mathrm{hd}}^{\mathrm{SM}}$ and $e_{E L F d}^{N}\left(n_{0}=n_{\mathrm{hd}}, n_{1}=n_{0}\right)=e_{\mathrm{hd}}^{\mathrm{NM}}$, giving

$$
\begin{aligned}
& v_{3}^{\text {is }}=6\left(e_{\mathrm{hd}}^{\mathrm{SM}}-t_{\mathrm{hd}}^{\mathrm{SM}}\right)-6 v_{0}^{\mathrm{is}}-6 v_{1}^{\mathrm{is}}-3 v_{2}^{\mathrm{is}}, \\
& v_{3}^{\mathrm{iv}}=-6\left(e_{\mathrm{hd}}^{\mathrm{SM}}-e_{\mathrm{hd}}^{\mathrm{NM}}-t_{\mathrm{hd}}^{\mathrm{SM}}+t_{\mathrm{hd}}^{\mathrm{NM}}\right)-6 v_{0}^{\mathrm{iv}}-6 v_{1}^{\mathrm{iv}}-3 v_{2}^{\mathrm{iv}},
\end{aligned}
$$

while considering $N=4$, the parameters $Q_{\text {sat } / \text { sym }}$ and $Z_{\text {sat } / \text { sym }}$ are given by $e_{E L F d}^{N}\left(n_{0}=n_{\mathrm{hd}}, n_{1}=0\right)=e_{\mathrm{hd}}^{\mathrm{SM}}, e_{E L F d}^{N}\left(n_{0}=n_{\mathrm{hd}}\right.$, $\left.n_{1}=n_{0}\right)=e_{\mathrm{hd}}^{\mathrm{NM}}, \quad$ and $\quad p_{E L F d}^{N}\left(n_{0}=n_{\mathrm{hd}}, n_{1}=0\right)=p_{\mathrm{hd}}^{\mathrm{SM}}$, $p_{E L F d}^{N}\left(n_{0}=n_{\mathrm{hd}}, n_{1}=n_{0}\right)=p_{\mathrm{hd}}^{\mathrm{NM}}$, where $e_{\mathrm{hd}}^{\mathrm{SM} / \mathrm{NM}}$ and $p_{\mathrm{hd}}^{\mathrm{SM} / \mathrm{NM}}$ are the energy per nucleon and pressure at the known reference point.
These conditions lead to the following expressions:

$$
\begin{aligned}
v_{3}^{\mathrm{is}}= & 24\left[e_{\mathrm{hd}}^{\mathrm{SM}}-t_{\mathrm{hd}}^{\mathrm{SM}}\right]-\frac{9}{8 n_{\mathrm{sat}}}\left[p_{\mathrm{hd}}^{\mathrm{SM}}-p_{\mathrm{hd}}^{\mathrm{kin}, \mathrm{SM}}\right] \\
& -6\left[4 v_{0}^{\mathrm{is}}+3 v_{1}^{\mathrm{is}}+v_{2}^{\mathrm{is}}\right] \\
v_{3}^{\mathrm{iv}}= & -24\left[e_{\mathrm{hd}}^{\mathrm{SM}}-e_{\mathrm{hd}}^{\mathrm{NM}}-t_{\mathrm{hd}}^{\mathrm{SM}}+t_{\mathrm{hd}}^{\mathrm{NM}}\right] \\
& +\frac{9}{8 n_{\mathrm{sat}}}\left[p_{\mathrm{hd}}^{\mathrm{SM}}-p_{\mathrm{hd}}^{\mathrm{NM}}-p_{\mathrm{hd}}^{\mathrm{kin}, \mathrm{SM}}+p_{\mathrm{hd}}^{\mathrm{kin}, \mathrm{NM}}\right] \\
& -6\left[4 v_{0}^{\mathrm{iv}}+3 v_{1}^{\mathrm{iv}}+v_{2}^{\mathrm{iv}}\right], \\
v_{4}^{\mathrm{is}}= & -72\left[e_{\mathrm{hd}}^{\mathrm{SM}}-t_{\mathrm{hd}}^{\mathrm{SM}}\right]+\frac{9}{2 n_{\mathrm{sat}}}\left[p_{\mathrm{hd}}^{\mathrm{SM}}-p_{\mathrm{hd}}^{\mathrm{kin}, \mathrm{SM}}\right] \\
& +12\left[6 v_{0}^{\mathrm{is}}+4 v_{1}^{\mathrm{is}}+v_{2}^{\mathrm{is}}\right], \\
v_{4}^{\mathrm{iv}}= & 72\left[e_{\mathrm{hd}}^{\mathrm{SM}}-e_{\mathrm{hd}}^{\mathrm{NM}}-t_{\mathrm{hd}}^{\mathrm{SM}}+t_{\mathrm{hd}}^{\mathrm{NM}}\right] \\
& -\frac{9}{2 n_{\mathrm{sat}}}\left[p_{\mathrm{hd}}^{\mathrm{SM}}-p_{\mathrm{hd}}^{\mathrm{NM}}-p_{\mathrm{hd}}^{\mathrm{kin}, \mathrm{SM}}+p_{\mathrm{hd}}^{\mathrm{kin}, \mathrm{NM}}\right] \\
& +12\left[6 v_{0}^{\mathrm{iv}}+4 v_{1}^{\mathrm{iv}}+v_{2}^{\mathrm{iv}}\right],
\end{aligned}
$$

where $p^{\mathrm{kin}}$ is the kinetic contribution to the pressure.

This high-density reference point $h d$ should ideally be taken from empirical information, such as might be given in the future by high-energy heavy-ion collisions with exotic beams. For the time being, such an empirical reference does not exist, and we will take for $e_{\mathrm{hd}}^{\mathrm{SM} / \mathrm{NM}}$ and $p_{\mathrm{hd}}^{\mathrm{SM} / \mathrm{NM}}$ the values given by a reference model. This introduces again some model dependence in the EOS, which is exactly what we want to avoid with the empirical treatment. To circumvent this problem, in the calculation of nuclear and astrophysical observables we will consider huge uncertainties for the high-order parameters from Table VI, such as to cover the whole domain of density dependence at high density. In this sense, the reference values for $Q_{\text {sat/sym }}, Z_{\text {sat/sym }}$ given by Eqs. (42)-(45) should only be considered as the central values of a large prior distribution which has to be filtered through constraints from astrophysical or laboratory observables.

The new metamodeling ELFd is shown in Fig. 6 and compared to the metamodeling ELFc for $N=3$ and 4 . We are using the nonrelativistic Skyrme SLy5 [132] and the RMF PK1 [68] as reference models for this illustration. We can see that the reference model is very accurately reproduced by ELFd already for $N=4$. A quantitative comparison of the metamodeling ELFd and other reference models is given in Sec. IV. The pressure and the sound velocity which account for first and second derivatives of the EOS are shown in Fig. 7, where metamodelings ELFc and ELFd are compared to the reference models for $N=3$ and 4 . An excellent agreement between metamodeling ELFd with $N=4$ with the reference model can be remarked. This is also reflected in the standard deviations between the binding energies predicted by the models and the ones of the metamodeling, which are shown in Table VI under the columns $\sigma_{e}$ in SM and NM.

To summarize, we have shown that, in order to better reproduce the density dependence of the binding energy and its derivatives up to about $4 n_{\text {sat }}$, it is necessary to readjust the empirical parameters $Q_{\text {sat/sym }}$ and $Z_{\text {sat } / \text { sym }}$ by explicitly introducing some EOS information at another reference density. 
TABLE VI. Modification of the empirical parameters, $Q_{\text {sat/sym }}$ and $Z_{\text {sat/sym }}$, in the metamodeling ELFd adjusted to reproduce reference models. The residual difference between the energy per particle given by the EOS and the one given by the associated metamodeling is shown as $\sigma_{e}$ in SM and NM. This distribution of the residual difference is encoded in terms of an average value and a standard deviation. It is evaluated between $n_{\text {sat }}$ and $4 n_{\text {sat }}$ considering 45 densities in total.

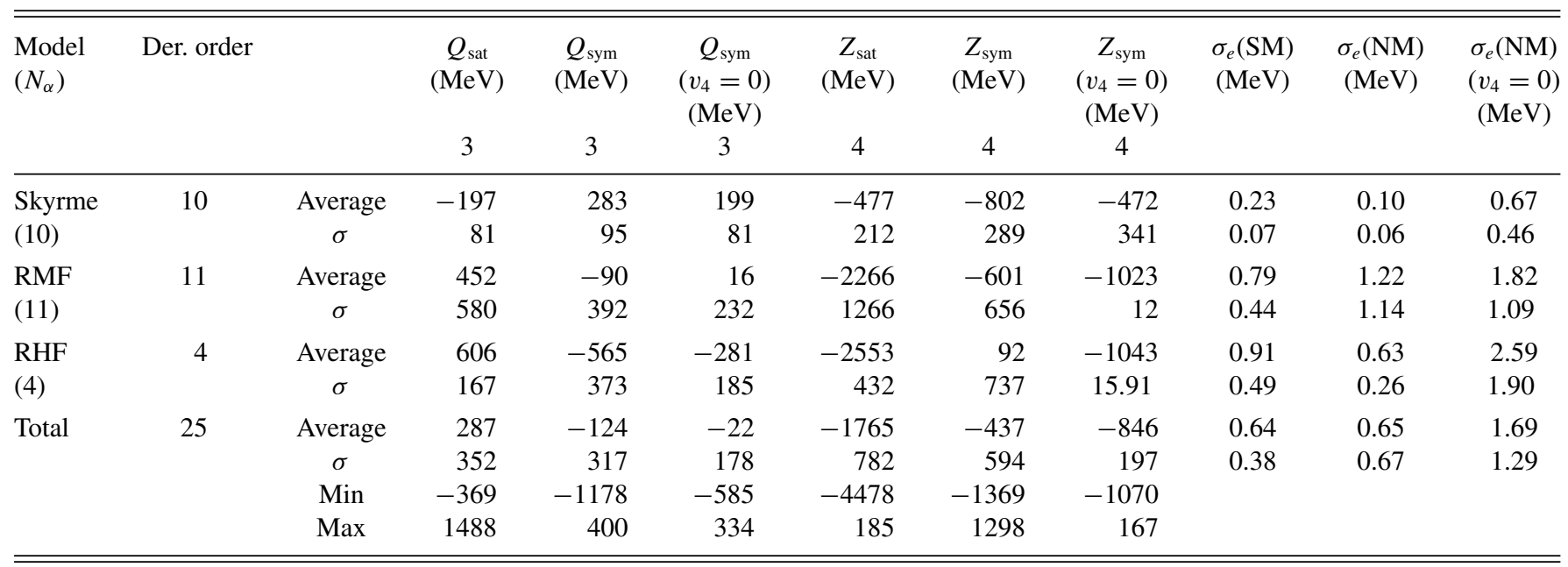

The introduction of this other reference density $n_{\text {hd }}$ stands for the necessity to complement the information determined at saturation density, and could potentially be obtained by different ways, such as for instance HIC or from the properties of NS. We have decided to take the reference density $n_{\text {hd }}=$ $4 n_{\text {sat }}$. It is indeed an arbitrary choice, made only to simplify equations (42)-(44), but we have checked that the final result is largely unaffected by the choice of the reference density, provided it is larger than $2-3 n_{\text {sat }}$.

More systematic comparisons and more quantitative criterion for the comparison between metamodeling ELFd and reference models shall now be presented.

\section{SYSTEMATICAL COMPARISON OF THE METAMODELING ELFD WITH EXISTING EOS}

Our final proposition for the meta-EOS is ELFd presented in Sec. IIIE. We now turn to show the main advantages of this metamodeling, namely (i) the model is sufficiently flexible to be able to reproduce most existing phenomenological and

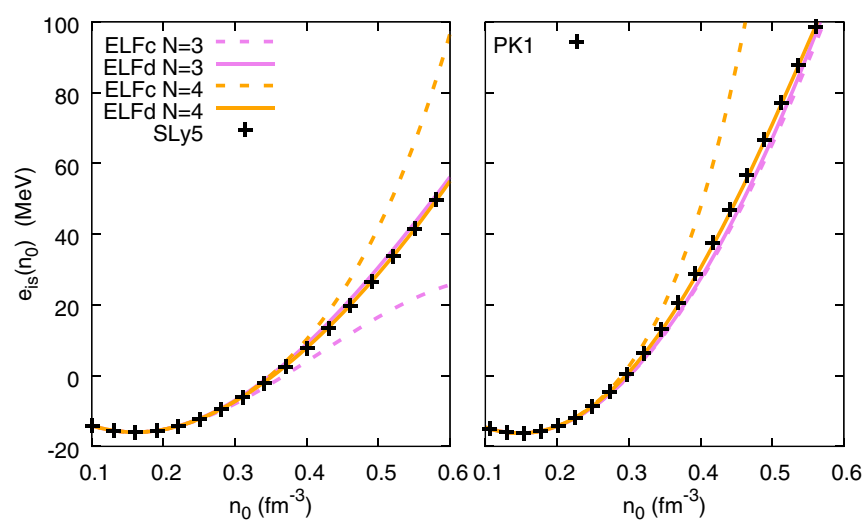

FIG. 6. Comparison of the ELFc (dashed lines) and ELFd (solid lines) metamodels against the reference models SLy5 (left panel) and PK1 (right panel). Only the orders $N=3$ and 4 are plotted. $a b$ initio functionals and (ii) it can also accommodate density dependences which might correspond to a physical behavior but are forbidden in the existing phenomenological EOS because of the assumed functional form.

\section{A. Extracting high-order parameters from the density behavior of existing analytical models}

In this section, the values of the empirical parameters $Q_{\text {sat/sym }}$ and $Z_{\text {sat/sym }}$ are calculated from Eqs. (42)-(45) for a large number of nuclear relativistic and nonrelativistic interactions. We ran over the same models as the one already considered in the study presented in Table IV, for instance. Detailed results are given in Table XIII and summarized in Table VI. In order to evaluate the effect induced by the highest order term in the series expansion, $v_{4}$, on the empirical parameters $Q_{\text {sym }}$ and $Z_{\text {sym }}$, we have added a column in Table VI where these empirical parameters are evaluated, imposing $v_{4}=0$. The last columns in Table VI stand for the average
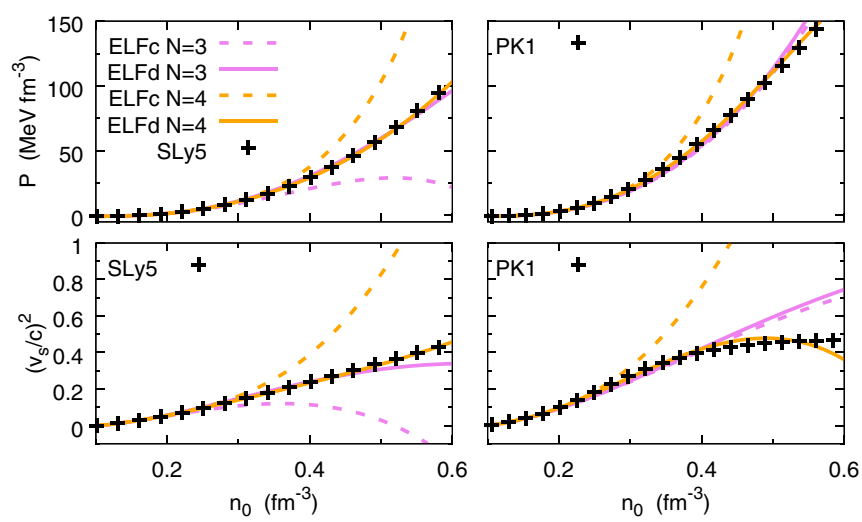

FIG. 7. Comparison of the pressure (top panels) and sound velocity (bottom panels) for the metamodels ELFc (dashed lines) and ELFd (solid lines) against the reference models SLy5 (left panels) and PK1 (right panels). Only the orders $N=3$ and 4 are plotted. 
TABLE VII. Synthesis of the expected values for the empirical parameters and their associated uncertainties. They are extracted from experimental analysis, except for the empirical parameters $Q_{\text {sat/sym }}$ and $Z_{\text {sat/sym }}$, which are estimated from Table VI.

\begin{tabular}{|c|c|c|c|c|c|c|c|c|c|c|c|c|}
\hline$P_{\alpha}$ & $\begin{array}{c}E_{\text {sat }} \\
(\mathrm{MeV})\end{array}$ & $\begin{array}{c}E_{\text {sym }} \\
(\mathrm{MeV})\end{array}$ & $\begin{array}{c}n_{\text {sat }} \\
\left(\mathrm{fm}^{-3}\right)\end{array}$ & $\begin{array}{c}L_{\text {sym }} \\
(\mathrm{MeV})\end{array}$ & $\begin{array}{c}K_{\text {sat }} \\
(\mathrm{MeV})\end{array}$ & $\begin{array}{c}K_{\text {sym }} \\
(\mathrm{MeV})\end{array}$ & $\begin{array}{c}Q_{\text {sat }} \\
(\mathrm{MeV})\end{array}$ & $\begin{array}{c}Q_{\text {sym }} \\
(\mathrm{MeV})\end{array}$ & $\begin{array}{c}Z_{\text {sat }} \\
(\mathrm{MeV})\end{array}$ & $\begin{array}{c}Z_{\mathrm{sym}} \\
(\mathrm{MeV})\end{array}$ & $m_{\text {sat }}^{*} / m$ & $\Delta m_{\text {sat }}^{*} / m$ \\
\hline$\left\langle P_{\alpha}\right\rangle$ & -15.8 & 32 & 0.155 & 60 & 230 & -100 & 300 & 0 & -500 & -500 & 0.75 & 0.1 \\
\hline$\sigma_{P_{\alpha}}$ & \pm 0.3 & \pm 2 & \pm 0.005 & \pm 15 & \pm 20 & \pm 100 & \pm 400 & \pm 400 & \pm 1000 & \pm 1000 & \pm 0.1 & \pm 0.1 \\
\hline
\end{tabular}

dispersion between the fitted EOS and the original data for symmetric and neutron matter, considering densities from $n_{\text {sat }}$ up to $4 n_{\text {sat }}$. It is particularly interesting to remark that the quality of EOS reproduction is very similar for relativistic and nonrelativistic models, even if we have employed a completely classical treatment of the kinetic energy density and no Dirac mass has been introduced.

In the isoscalar channel, it is interesting to remark that the parameter $Q_{\text {sat }}$ is systematically shifted up by about $200 \mathrm{MeV}$, and the average value of the parameter $Z_{\text {sat }}$ is shifted down by a large amount. There is therefore a compensation between the parameters $Q_{\text {sat }}$ and $Z_{\text {sat }}$. In absolute value the parameters $Z_{\text {sat }}$ in Table VI are lower than in Table IV, and the issue related to alternative series is now pushed to densities well above $4 n_{\text {sat }}$, where our approach is not any more well suited.

In the isovector channel, both $Q_{\text {sym }}$ and $Z_{\text {sym }}$ are shifted down and in absolute value the parameters $Z_{\text {sym }}$ in Table VI are lower than in Table IV, in the same way as for $Z_{\text {sat }}$. The issues induced by alternative series expansion are also reduced in NM.

The average dispersion in symmetric and neutron matter given in Table VI shows the excellent reproduction of the original EOS up to $4 n_{\text {sat }}$. It also shows the important role of $v_{4}$ since removing it shifts up the average standard deviation. In conclusion, Table VI shows that by considering the shifted value given in Table VI and Eqs. (42)-(44) it is possible to reproduce very accurately all the tested EOS with the ELFd metamodeling.

In addition to having proven that all the considered EOS can be well reproduced by the ELFd metamodel, we can use the average values given in Table VI to estimate the average values and uncertainties of the highest order empirical parameters $Q_{\text {sat/sym }}$ and $Z_{\text {sat } / \text { sym }}$. In Table VII, we present a global summary of all empirical parameters and estimated uncertainties based on our analysis presented in previous sections. The low-order empirical parameters $E_{\mathrm{sat} / \mathrm{sym}}, L_{\mathrm{sym}}$, and $K_{\text {sat } / \text { sym }}$ as well as the effective masses are compatible with the experimental analysis presented in Sec. II A and model average predictions in Sec. IIB, and synthesized in Tables IV and V. For the highest order empirical parameters $Q_{\text {sat/sym }}$ and $Z_{\text {sat/sym }}$, we have considered the shifted values given in Table VI with large estimated uncertainties $\sigma_{P_{\alpha}}$. The large estimated uncertainties are chosen such as to compensate the fact that the average values taken for these empirical parameters are only determined from the existing models, without any empirical information. The average value of $Z_{\text {sat }}$ is taken identical to $Z_{\text {sym }}$ for simplicity. In the following, we consider average values and uncertainties for the empirical parameters which are summarized in Table VII.

\section{B. Extracting empirical parameters from chiral EFT results}

Neutron matter calculations based on modern potentials have recently been performed up to saturation density; see, for instance, Refs. [11,12,126]. In these approaches, either advanced many-body techniques have been employed, such as auxiliary field diffusion Monte Carlo (AFDMC) with hardcore potentials [126] or with soft potentials from chiral EFT [13-15], or many-body perturbation theory based on chiral EFT [11,12]. The perturbative convergence of the chiral potential can be studied in detail in this approach and the equation of state is usually provided within a band representing the uncertainties in the nuclear interaction as well as in the convergence in the expansion. In this section, we perform an analysis of these recent predictions, based either on the fits provided by the authors or on their numerical results.

Considering the accurate fit for the energy per nucleon in neutron matter provided in Ref. [126],

$$
e\left(n_{0}\right)=a\left(\frac{n_{0}}{n_{\mathrm{sat}}}\right)^{\alpha}+b\left(\frac{n_{0}}{n_{\mathrm{sat}}}\right)^{\beta},
$$

where the parameters $a, \alpha, b$, and $\beta$ are provided in Table I of Ref. [126] for various prescriptions for the three-body force $(3 \mathrm{BF})$, we have calculated the empirical parameters in neutron matter at the fixed density $n_{\mathrm{sat}}=0.16 \mathrm{fm}^{-3}$ : $E_{\mathrm{NM}}=E_{\mathrm{sat}}+E_{\mathrm{sym}}$ (energy per nucleon), $L_{\mathrm{sym}}$ (slope of the symmetry energy), $K_{\mathrm{NM}}=K_{\text {sat }}+K_{\text {sym }}$ (curvature), $Q_{\mathrm{NM}}=$ $Q_{\text {sat }}+Q_{\text {sym }}$ (skewness), and $Z_{\mathrm{NM}}=Z_{\text {sat }}+Z_{\text {sym }}$ (kurtosis). These quantities are given in Table VIII for the seven different Hamiltonians explored in Ref. [126]. As in previous analyses, we have also extracted an average value and standard deviation for these seven Hamiltonians.

We have also analyzed the results for the first complete $\mathrm{N}^{3} \mathrm{LO}$ calculation of the neutron matter energy [11]. In this case, the energy and the pressure in neutron matter are provided as tabulated numbers with error bars, up to the density $n_{0}=$ $0.2 \mathrm{fm}^{-3}$. We have here considered the metamodeling ELFc with $N=2$ since only a small domain of density is provided (the empirical parameters associated to $N=3$ and 4 are undetermined by the provided data). To extract the empirical parameters and their error bars from these results, we have associated to each set of empirical parameters $\left(E_{\mathrm{NM}}, L_{\mathrm{sym}}, K_{\mathrm{NM}}\right)$ a $\chi^{2}$ defined as

$$
\begin{aligned}
\chi^{2}= & \frac{1}{2 M-3} \sum_{i=1}^{M}\left(\frac{e_{i}-e_{E L F c}\left(n_{0}^{i}\right)}{\epsilon_{i}^{e}}\right)^{2} \\
& +\left(\frac{p_{i}-p_{E L F c}\left(n_{0}^{i}\right)}{\epsilon_{i}^{p}}\right)^{2}
\end{aligned}
$$


TABLE VIII. Neutron matter energy per nucleon $E_{\mathrm{NM}}$, slope of the symmetry energy $L_{\text {sym }}$, incompressibility $K_{\mathrm{NM}}, Q_{\mathrm{NM}}$, and kurtosis $Z_{\mathrm{NM}}$ for the seven Hamiltonians studied in Ref. [126]. These Hamiltonians are treated within the AFDMC many-body method and they are based on AV8' NN potential supplemented by various three-body forces (3BF). They are named GCR in reference to the names of the authors.

\begin{tabular}{lcccccr}
\hline \hline Model & $3 \mathrm{BF}$ & $\begin{array}{c}E_{\mathrm{NM}} \\
(\mathrm{MeV})\end{array}$ & $\begin{array}{c}L_{\mathrm{sym}} \\
(\mathrm{MeV})\end{array}$ & $\begin{array}{c}K_{\mathrm{NM}} \\
(\mathrm{MeV})\end{array}$ & $\begin{array}{c}Q_{\mathrm{NM}} \\
(\mathrm{MeV})\end{array}$ & $\begin{array}{c}Z_{\mathrm{NM}} \\
(\mathrm{MeV})\end{array}$ \\
\hline GCR-1 & none & 14.48 & 30.7 & 17 & 55 & -117 \\
GCR-2 & $V_{2 \pi}^{\mathrm{PW}}+V_{\mu=150}^{R}$ & 16.15 & 40.2 & 45 & 52 & -117 \\
GCR-3 & $V_{2 \pi}^{\mathrm{PW}}+V_{\mu=300}^{R}$ & 15.99 & 39.8 & 47 & 59 & -121 \\
GCR-4 & $V_{3 \pi}+V_{R}$ & 16.21 & 42.9 & 76 & 93 & -137 \\
GCR-5 & $V_{2 \pi}^{\mathrm{PW}}+V_{\mu=150}^{R}$ & 17.76 & 50.8 & 82 & 56 & -121 \\
GCR-6 & $V_{3 \pi}+V_{R}$ & 17.71 & 54.7 & 128 & 121 & -149 \\
GCR-7 & $\mathrm{UIX}$ & 19.02 & 61.7 & 147 & 122 & -155 \\
Average & & 16.76 & 45.8 & 77 & 80 & -131 \\
$\sigma$ & & 1.39 & 9.7 & 43 & 29 & 15 \\
Min & & 14.48 & 30.7 & 17 & 52 & -155 \\
Max & & 19.02 & 61.7 & 147 & 122 & -117 \\
\hline \hline
\end{tabular}

where $\left(e_{i}, \epsilon_{i}^{e}\right)$ and $\left(p_{i}, \epsilon_{i}^{p}\right)$ are the average values and error bars for the energies and pressures predicted in Ref. [11]. A likelihood probability is then associated to each set of empirical parameters, as $p\left(E_{\mathrm{NM}}, L_{\mathrm{sym}}, K_{\mathrm{NM}}\right)=\exp \left(-\chi^{2} / 2\right) / p_{\text {norm }}$, and the distance to the maximum likelihood probability $\left(p_{\max }\right)$ is measured in terms of $\sigma$, where the models at $k \sigma$ are those for which $p / p_{\max }>\exp \left(-k^{2} / 2\right)$.

The $1 \sigma, 2 \sigma$, and $3 \sigma$ domains for ELFc meta-EOS passing through the binding energies and pressures calculated in Ref. [11] are represented in Fig. 8, while the representation of the probability distribution showing the correlations among the empirical parameters is shown in the corner of Fig. 9. The two-variable probabilities are defined as $p(A, B)=$ $\sum_{C} p(A, B, C)$, while the single-variable probabilities are $p(A)=\sum_{B, C} p(A, B, C)$, where $(A, B, C)$ can be any permutation of $\left(E_{\mathrm{NM}}, L_{\mathrm{sym}}, K_{\mathrm{NM}}\right)$.

It is interesting to note the nice correlation between $E_{\mathrm{NM}}$ and $L_{\text {sym }}$, as well as between $K_{\mathrm{NM}}$ and $L_{\mathrm{sym}}$, while $K_{\mathrm{NM}}$ is
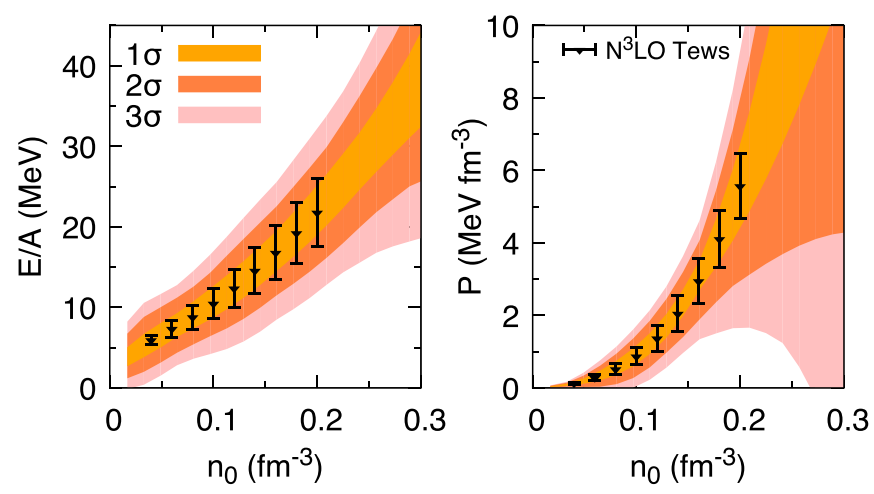

FIG. 8. Equations of state at $1 \sigma, 2 \sigma$, and $3 \sigma$ obtained from the first calculation of neutron matter with chiral EFT at next-to-next-to-next leading order $\left(\mathrm{N}^{3} \mathrm{LO}\right)$ (points with error bars) and given in Ref. [11]. See text for more details.

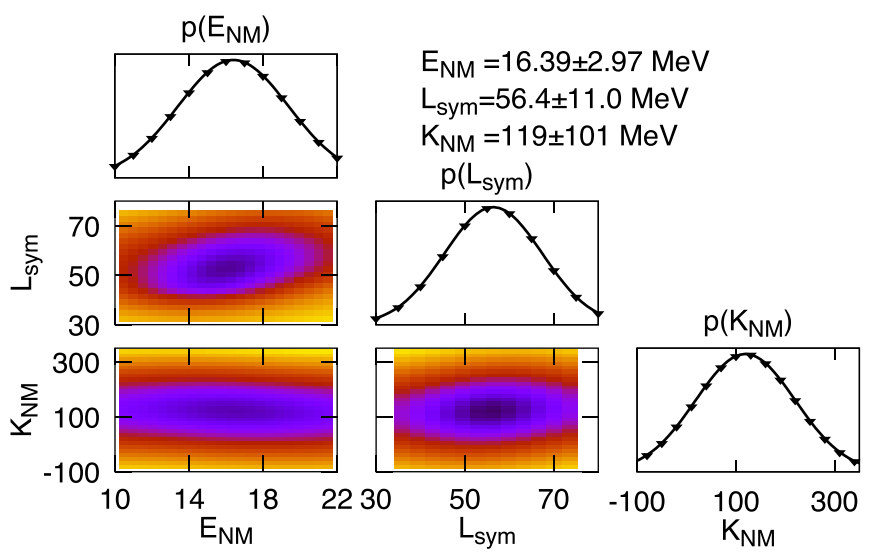

FIG. 9. Analysis of the probability distribution $p\left(E_{\mathrm{NM}}, L_{\mathrm{sym}}\right.$, $K_{\mathrm{NM}}$ ) deduced from the best fits to neutron matter calculations shown in Fig. 8. The centroids and standard deviations for the parameters $E_{\mathrm{NM}}, L_{\mathrm{sym}}$, and $K_{\mathrm{NM}}$ are given in the figure. See text for more details.

almost independent of $E_{\mathrm{NM}}$. The single-variable probabilities are shown on the diagonal of the corner in Fig. 9. The value of the centroids, defined as $\langle A\rangle=\sum_{A} A p(A) / \sum_{A} p(A)$ and of the standard deviation, defined as $\sigma_{A}=\sqrt{\left\langle A^{2}\right\rangle-\langle A\rangle^{2}}$, are given in Fig. 9 as well as in Table V.

Let us remember that the following constraints have been obtained in Ref. [11]: $E_{\text {sym }}=31.9 \pm 3 \mathrm{MeV}$ and $L_{\text {sym }}=$ $54.5 \pm 11.5 \mathrm{MeV}$. Assuming $E_{\text {sat }}=-16.0 \pm 0.5 \mathrm{MeV}$, we deduce $E_{\mathrm{NM}}=15.9 \pm 3.5 \mathrm{MeV}$ from Ref. [11]. These results are compatible with the ones we obtain from a different analysis. In addition, we could obtain a constraint for the neutron matter incompressibility $K_{\mathrm{NM}}$ which was not given in Ref. [11]. Let us finally remark that the determination of the parameter $K_{\mathrm{NM}}$ is possible since the pressure was introduced into the definition of the $\chi^{2}$. With the energies only, we would have obtained a much larger spread in the probability distribution of $K_{\mathrm{MN}}$. The fit of the energy per nucleon and pressure at the same time offers therefore very interesting constraints for the nuclear equation of state and its derivatives.

Finally, we analyze the very recent calculations of asymmetric nuclear matter based on chiral two- and three-body interactions at $\mathrm{N}^{3} \mathrm{LO}$ [12]. The empirical parameters shown in Table IX are deduced from the fit of the binding energy given in Ref. [12],

$$
e\left(n_{0}, n_{1}\right)=\sum_{\nu=2,3,4,5,6}\left(C_{0 v}+C_{2 v} \delta^{2}\right)\left(\frac{n_{0}}{0.16}\right)^{v / 3},
$$

where the values of the parameters $C_{0 v}$ and $C_{2 v}$ are given in Table II for the seven Hamiltonians detailed in Table I of Ref. [12].

The reported ranges for $K_{\text {sat }}$ and $E_{\text {sym }}$ in Ref. [12] are very close to ours, $K_{\text {sat }}=218 \pm 36 \mathrm{MeV}$ and $E_{\text {sym }}=32.05 \pm$ 3.65 MeV, if we consider the values we obtained from the minimal (Min) and maximal (Max) values for these parameters. The slight difference we obtained may come from the fact that the free Hartree-Fock spectrum was also considered in Ref. [12] for the estimation of the error bars. 
TABLE IX. Binding energy $E_{\text {sat }}$, the symmetry energy $E_{\text {sym }}$, saturation density $n_{\text {sat }}$, slope of the symmetry energy $L_{\text {sym }}$, isoscalar incompressibility $K_{\text {sat }}$, isovector incompressibility $K_{\text {sym }}$, isoscalar skewness $Q_{\text {sat }}$, isovector skewness $Q_{\text {sym }}$, isoscalar kurtosis $Z_{\text {sat }}$, isovector kurtosis $Z_{\text {sym }}$, and $K_{\tau}$ for chiral EFT given in Ref. [12].

\begin{tabular}{|c|c|c|c|c|c|c|c|c|c|c|c|}
\hline Model & $\begin{array}{c}E_{\mathrm{sat}} \\
(\mathrm{MeV})\end{array}$ & $\begin{array}{c}E_{\text {sym }} \\
(\mathrm{MeV})\end{array}$ & $\begin{array}{c}n_{\mathrm{sat}} \\
\left(\mathrm{fm}^{-3}\right)\end{array}$ & $\begin{array}{c}L_{\text {sym }} \\
(\mathrm{MeV})\end{array}$ & $\begin{array}{c}K_{\text {sat }} \\
(\mathrm{MeV})\end{array}$ & $\begin{array}{c}K_{\text {sym }} \\
(\mathrm{MeV})\end{array}$ & $\begin{array}{c}Q_{\text {sat }} \\
(\mathrm{MeV})\end{array}$ & $\begin{array}{c}Q_{\text {sym }} \\
(\mathrm{MeV})\end{array}$ & $\begin{array}{c}Z_{\text {sat }} \\
(\mathrm{MeV})\end{array}$ & $\begin{array}{c}Z_{\text {sym }} \\
(\mathrm{MeV})\end{array}$ & $\begin{array}{c}K_{\tau} \\
(\mathrm{MeV})\end{array}$ \\
\hline 1 & -16.92 & 34.57 & 0.189 & 48.5 & 241 & -224 & -125 & -311 & 1281 & -1974 & -490 \\
\hline 3 & -15.18 & 32.18 & 0.174 & 53.0 & 224 & -108 & 24 & 96 & 1299 & -2961 & 432 \\
\hline 4 & -14.84 & 31.63 & 0.170 & 46.1 & 198 & -170 & -223 & -82 & 1534 & -2464 & -395 \\
\hline 5 & -13.80 & 29.83 & 0.158 & 44.5 & 182 & -143 & -310 & 69 & 1537 & -2638 & -334 \\
\hline Average & -15.16 & 32.01 & 0.171 & 48.1 & 214 & -172 & -139 & -164 & 1306 & -2317 & -428 \\
\hline$\sigma$ & 1.24 & 2.09 & 0.016 & 3.6 & 22 & 40 & 104 & 234 & 214 & 379 & 63 \\
\hline Min & -16.92 & 28.53 & 0.140 & 43.9 & 182 & -224 & -310 & -640 & 901 & -2961 & -534 \\
\hline Max & -13.23 & 34.57 & 0.190 & 53.5 & 242 & -108 & 24 & 96 & 1537 & -1750 & -334 \\
\hline
\end{tabular}

The empirical parameters of neutron matter given in Table $\mathrm{V}$ are obtained from the combining of isoscalar and isovector parameters given in Table IX.

\section{Behavior of the symmetry energy around saturation density}

In the previous sections, we have shown that our meta-EOS can very accurately reproduce existing models of very different types (relativistic and nonrelativistic, phenomenological and $a b$ initio). It can also be used to make complete statistical analysis of such models, for example, to evaluate the confidence interval of a nuclear or astrophysical observable compatible with chiral EFT at any chosen confidence level. We now turn to show that the parameter space is large enough to accommodate density behaviors which cannot be explored by existing functionals. To this aim, we will take the example of the density dependence of the symmetry energy.

Many theoretical and experimental studies of the density dependence of the symmetry energy consider models with very limited number of parameters. This leads to some strong correlations in the density dependence of the symmetry energy, e.g., isosoft behavior below $n_{\text {sat }}$ and isostiff behavior above $n_{\text {sat }}$. We have discussed, for instance, the opposite impact of $L_{\text {sym }}$ and $K_{\text {sym }}$ in the analysis of Figs. 12 and 13. Combining together the effect of $L_{\text {sym }}$ and $K_{\text {sym }}$ a rich behavior for the symmetry energy can be explored.

We illustrate our purpose in Fig. 10 where the symmetry energy $S\left(n_{0}\right)$ is represented in various cases: $K_{\text {sym }}$ is varied for fixed values of $L_{\text {sym }}$ (top panels), and $L_{\text {sym }}$ is varied for fixed values of $K_{\text {sym }}$ (bottom panels). We observe on the top
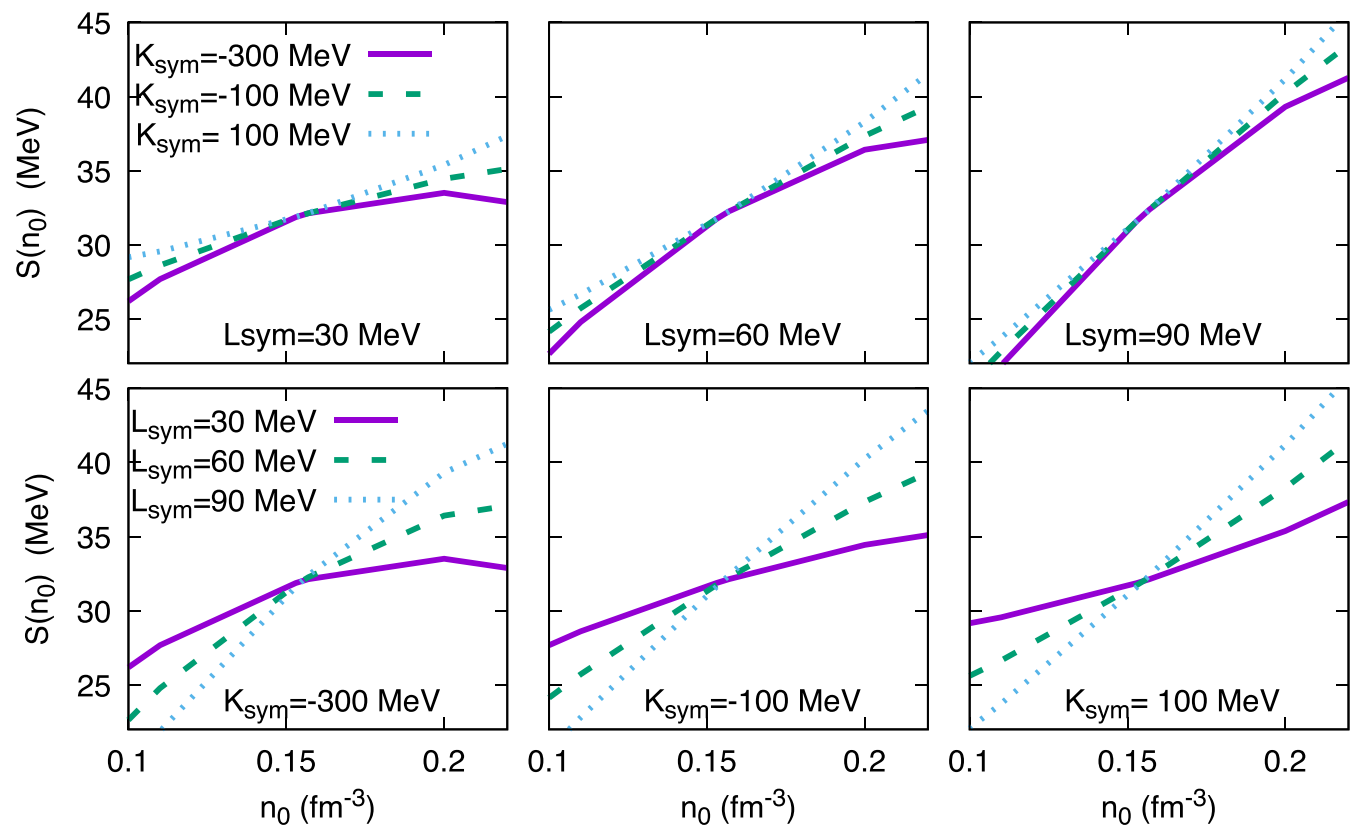

FIG. 10. Effect of varying the value of $L_{\text {sym }}$ and $K_{\text {sym }}$ on the symmetry energy around saturation density. Note that we vary the parameters by $\pm 2 \sigma$ to enhance the effects. Top panels: varying $K_{\text {sym }}$ at fixed $L_{\text {sym }}$. Bottom panels: varying $L_{\text {sym }}$ at fixed $K_{\text {sym }}$. 

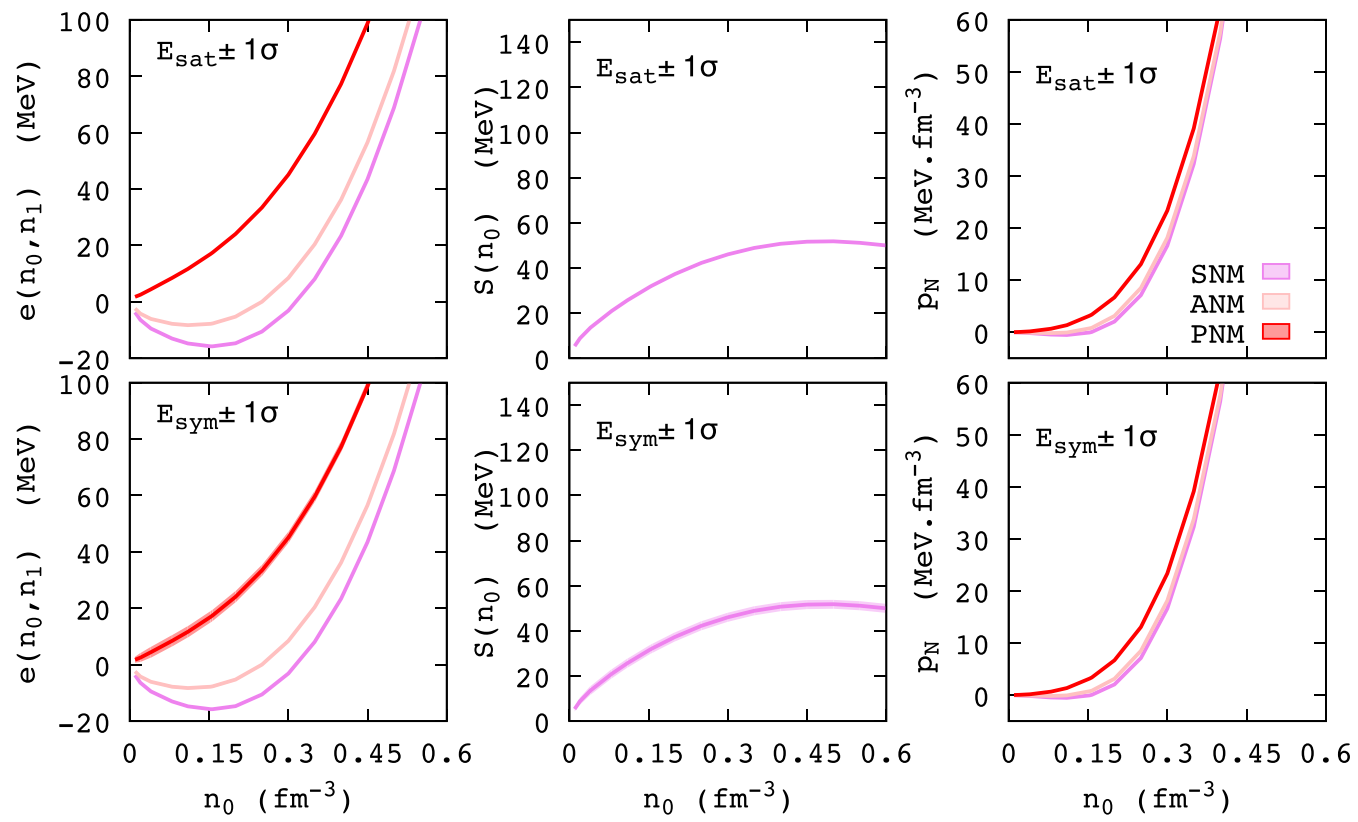

FIG. 11. Effect of varying the value of $E_{\text {sat }}$ (top panels) and $E_{\text {sym }}$ (bottom panels) around the mean value given in Tab. VII considering $1 \sigma$ deviation. Here $1 \sigma$ is also taken from Tab. VII. From left to right: energy per nucleon, symmetry energy and pressure in SNM $(\delta=0)$, ANM $(\delta=0.5)$, and $\operatorname{PNM}(\delta=1)$.

panels of Fig. 10 that for a fixed value of $L_{\text {sym }}$, changing the value of $K_{\text {sym }}$ changes the curvature of the symmetry energy around $n_{\text {sat }}$ : Negative values of $K_{\text {sym }}$ produce a concave density dependence of the symmetry energy, e.g., isosoft below $n_{\text {sat }}$ and isosoft above $n_{\text {sat }}$, while positive values of $K_{\text {sym }}$ produce a convex density dependence of the symmetry energy, e.g., isostiff below $n_{\text {sat }}$ and isostiff above $n_{\text {sat }}$. We now turn to the bottom panels of Fig. 10 where we have varied $L_{\text {sym }}$ at fixed value of $K_{\text {sym }}$. Varying $L_{\text {sym }}$ generates different slopes of the symmetry energy which are more or less isostiff below $n_{\text {sat }}$ and isosoft above $n_{\text {sat }}$.

In conclusion, we have shown that it is possible to play with the two most important empirical parameters $L_{\text {sym }}$ and $K_{\text {sym }}$ to modify the behavior of the symmetry energy around $n_{\text {sat }}$ and explore a wide range of possible density dependence.

\section{EFFECT OF THE PRESENT UNCERTAINTIES ON THE NUCLEAR EOS}

In this section, we will explore another advantage of the proposed formalism, namely the possibility of studying the effect of the different empirical parameters independently from each other. This allows performing a sensitivity study on the meta-EOS and recognizing the most influential empirical parameters.

We consider Table VII as a reference for fixing an average nuclear meta-EOS and probing the impact of varying the empirical parameters within their estimated uncertainties.

\section{A. Effect of varying a single parameter}

In this analysis, we define an average meta-EOS determined by the average empirical parameters given in Table VII, then we vary the empirical parameters one after the other to estimate the impact of the estimated uncertainties on the prediction of the EOS. This impact is obtained as a combined effect between the real influence of the considered parameter and its estimated uncertainty. For instance, an influential empirical parameter known very accurately will have a weak influence, while a less influential but very poorly known empirical parameter might have a large influence. In the following, we will also observe that the different order empirical parameters play a role a different densities. In general, as the order is higher, the farther from saturation density it has an impact.

In Figs. 11-16, we have grouped together the isoscalar and isovector empirical parameters order by order. On the top panels are shown the effects induced by the change of the isoscalar empirical parameters, while on the bottom panels it is the impact isovector empirical parameters which are tested.

Let us start with Fig. 11, where we show the impact of varying $E_{\text {sat } / \text { sym }}$ considering a $1 \sigma$ deviation around the average meta-EOS given in Table VII. The energy per particle and the pressure for SNM, ANM (defined as $\delta=0.5$ ), and PNM are shown as a function of the density, and the symmetry energy $S\left(n_{0}\right)$ is also represented in Fig. 11. The impact of our uncertainty on $E_{\text {sat/sym }}$ is rather weak for the energy per particle, the symmetry energy, and the pressure. Let us note that since the pressure is the derivative of the energy per particle, it is not impacted at all by $E_{\text {sat } / \text { sym }}$.

More interestingly we show in Fig. 12 the impact of varying $n_{\text {sat }}$ and $L_{\text {sym }}$ in the same way as in Fig. 11. The impact of our uncertainty on $n_{\text {sat }}$ is quite small, while the effect of varying $L_{\text {sym }}$ has a noticeable impact above and below saturation density for the energy per particle, the symmetry energy, and the pressure. In asymmetric and neutron matter, the effect of $L_{\text {sym }}$ below saturation density is opposite to above saturation density. So a large value of $L_{\text {sym }}$ will strongly reduce (respectively, increase) the energy per particle below (respectively, above) 

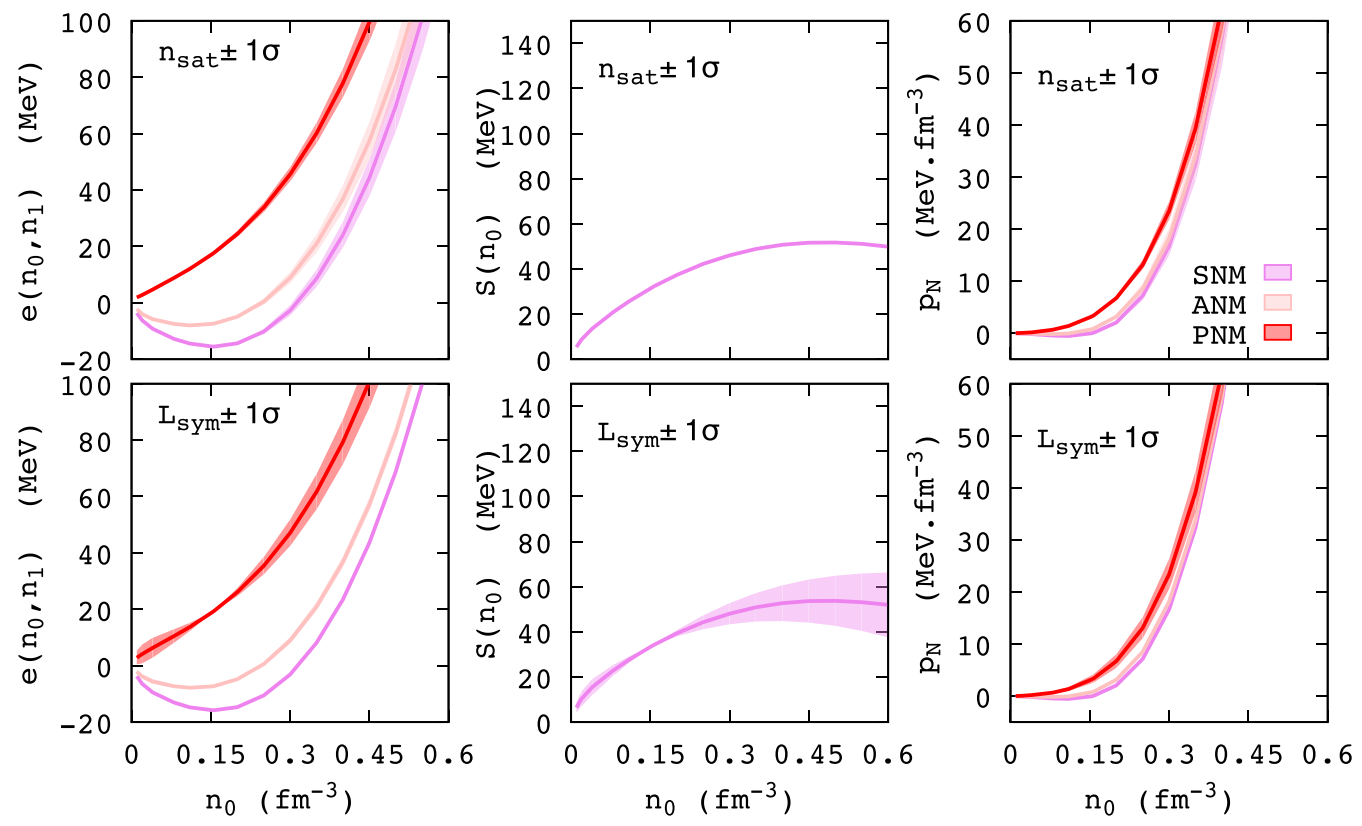

FIG. 12. Same as Fig. 11 for $n_{\text {sat }}$ (top panel) and $L_{\text {sym }}$ (bottom panel).

saturation density. The crossing as saturation density of these quantities is expected since the meta-EOS is a series expansion taking $n_{\text {sat }}$ as the reference density. The slope of the symmetry energy is sensitive to $L_{\text {sym }}$. The largest value of $L_{\text {sym }}$ that we have considered induces a positive slope until $n_{\text {sat }}$ while the lowest value produce a negative slope. For the pressure, since it is obtained as the derivative of the energy per particle, the uncertainty in $L_{\text {sym }}$ propagates almost constantly through the densities.

The impact of our uncertainty in $K_{\text {sat } / \text { sym }}$ is shown in Fig. 13. While the uncertainty in the value of $K_{\text {sat }}$ has a weak impact on the nuclear EOS (almost unnoticeable), the large uncertainty of
$K_{\text {sym }}$ has a large impact on the nuclear EOS. At variance with $L_{\text {sym }}$, the effect of $K_{\text {sym }}$ below and above saturation density is similar: an increase of $K_{\text {sym }}$ increases the energy per particle both below and above $n_{\text {sat }}$. Large negative values of $K_{\text {sym }}$ force the symmetry energy $S$ to bend down at high density. For very large values of $K_{\mathrm{sym}}, S$ could almost become negative below $4 n_{\text {sat }} . K_{\text {sym }}$ is therefore the most influential empirical parameter governing the density dependence of the symmetry energy from $2 n_{\text {sat }}$ to $4 n_{\text {sat }}$.

The impact of the variation of $Q_{\text {sat } / \text { sym }}$ is shown in Fig. 14 . The uncertainty in $Q_{\text {sat }}$ has a quite large impact on the EOS and this is clearly the most important empirical parameter in the
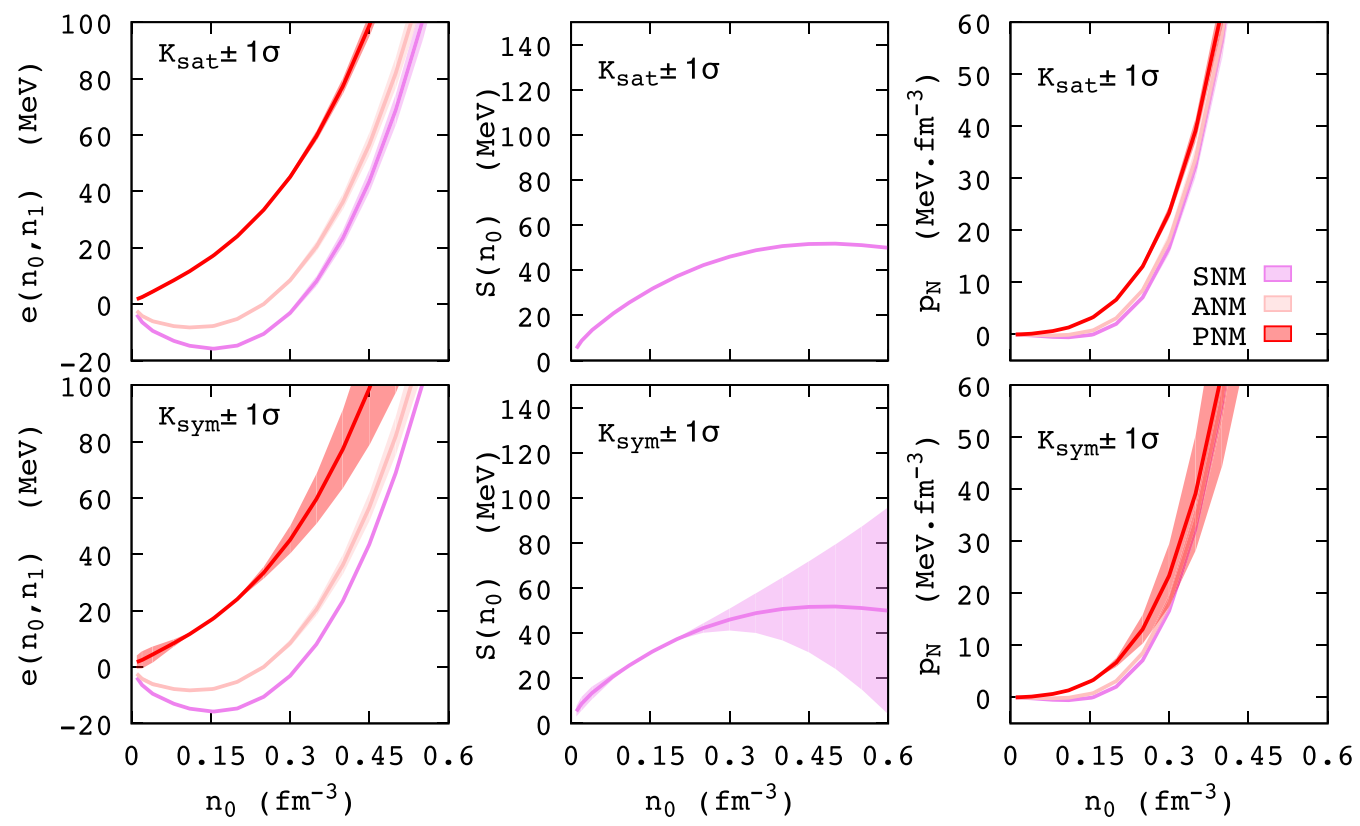

FIG. 13. Same as Fig. 11 for $K_{\text {sat }}$ (top panel) and $K_{\text {sym }}$ (bottom panel). 

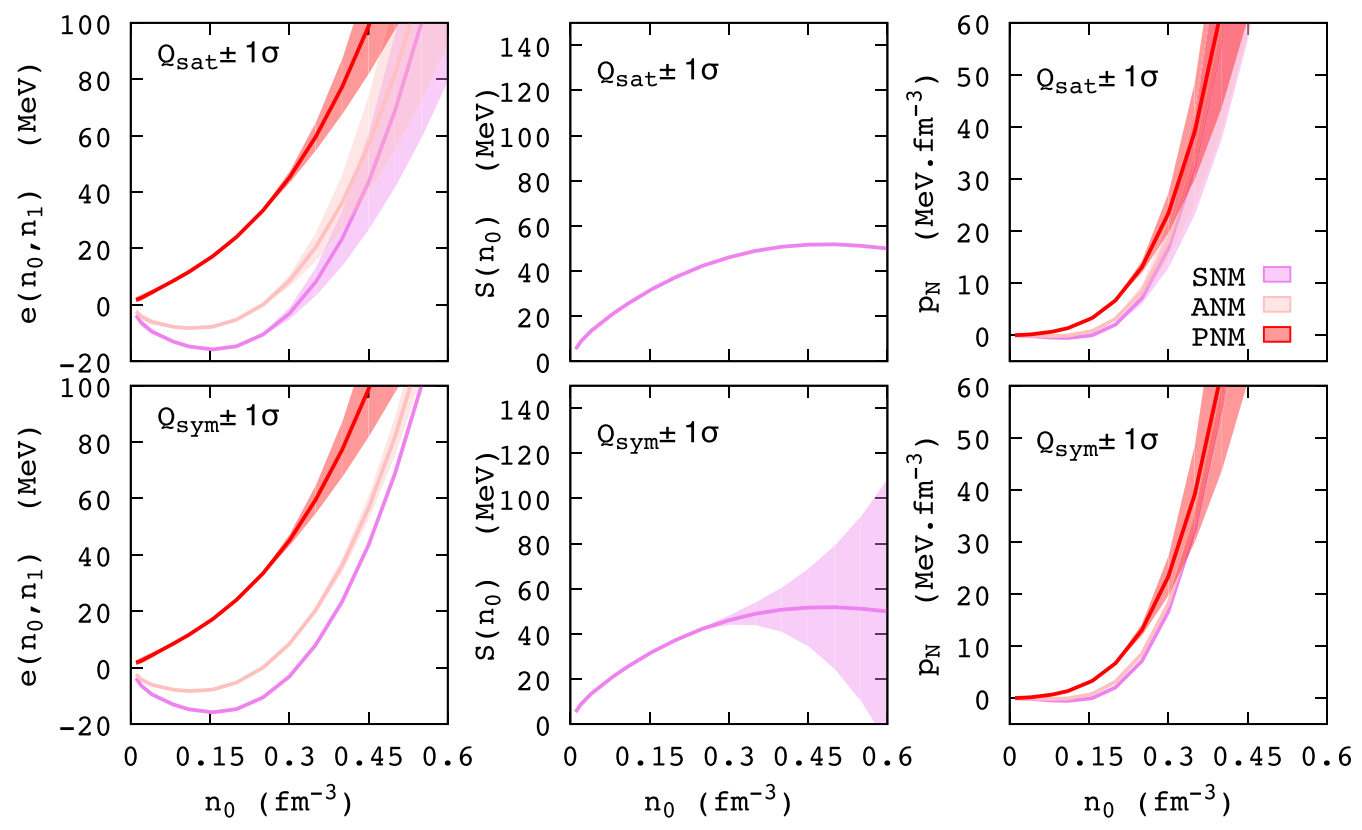

FIG. 14. Same as Fig. 11 for $Q_{\text {sat }}$ (top panel) and $Q_{\text {sym }}$ (bottom panel).

isoscalar channel which is yet weakly known. The uncertainty in $Q_{\text {sym }}$ has also an important impact on the EOS. Its impact starts above $2 n_{\text {sat }}$.

We show in Fig. 15 the impact of our uncertainty in the parameters $Z_{\text {sat/sym }}$. Both $Z_{\text {sat }}$ and $Z_{\text {sym }}$ have an impact on the EOS. By comparing Figs. 12 to 15 , it is interesting to observe that as the empirical parameter is higher, the farther from $n_{\text {sat }}$ it has an impact. This effect is limited by the increasing uncertainty in the empirical parameters as their order increases, but nevertheless it is rather visible. It is a consequence of the series expansion since the different order terms in the series expansion have a weight which goes decreasing around saturation density as their order increases.

The last figure of this series, Fig. 16, shows the impact of the isoscalar and isovector splitting of the Landau effective mass, e.g., $m_{\text {sat }}^{*}$ and $\Delta m_{\text {sat }}^{*}$. It is very interesting to observe that the Landau effective masses $m_{\text {sat }}^{*}$ and $\Delta m_{\text {sat }}^{*}$ have a very weak impact on the EOS. We remember that in our EOS, we are able to probe the impact of each empirical parameter separately, leaving the other parameters unchanged. It is clear from Fig. 16 that the uncertainties in the Landau effective mass are very limited and almost unobservable for the EOS. It is, however, expected to impact more crucially the single-particle properties
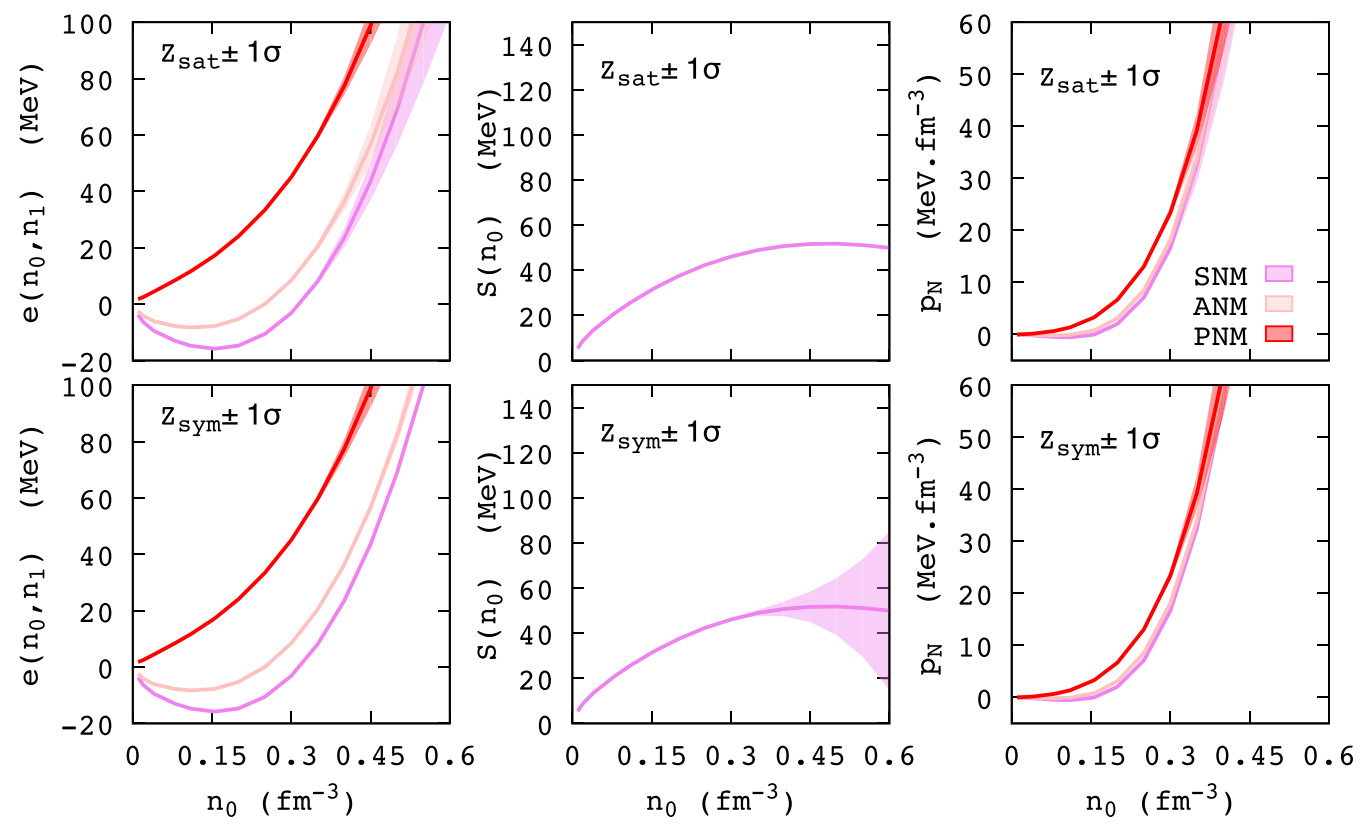

FIG. 15. Same as Fig. 11 for $Z_{\text {sat }}$ (top panel) and $Z_{\text {sym }}$ (bottom panel). 

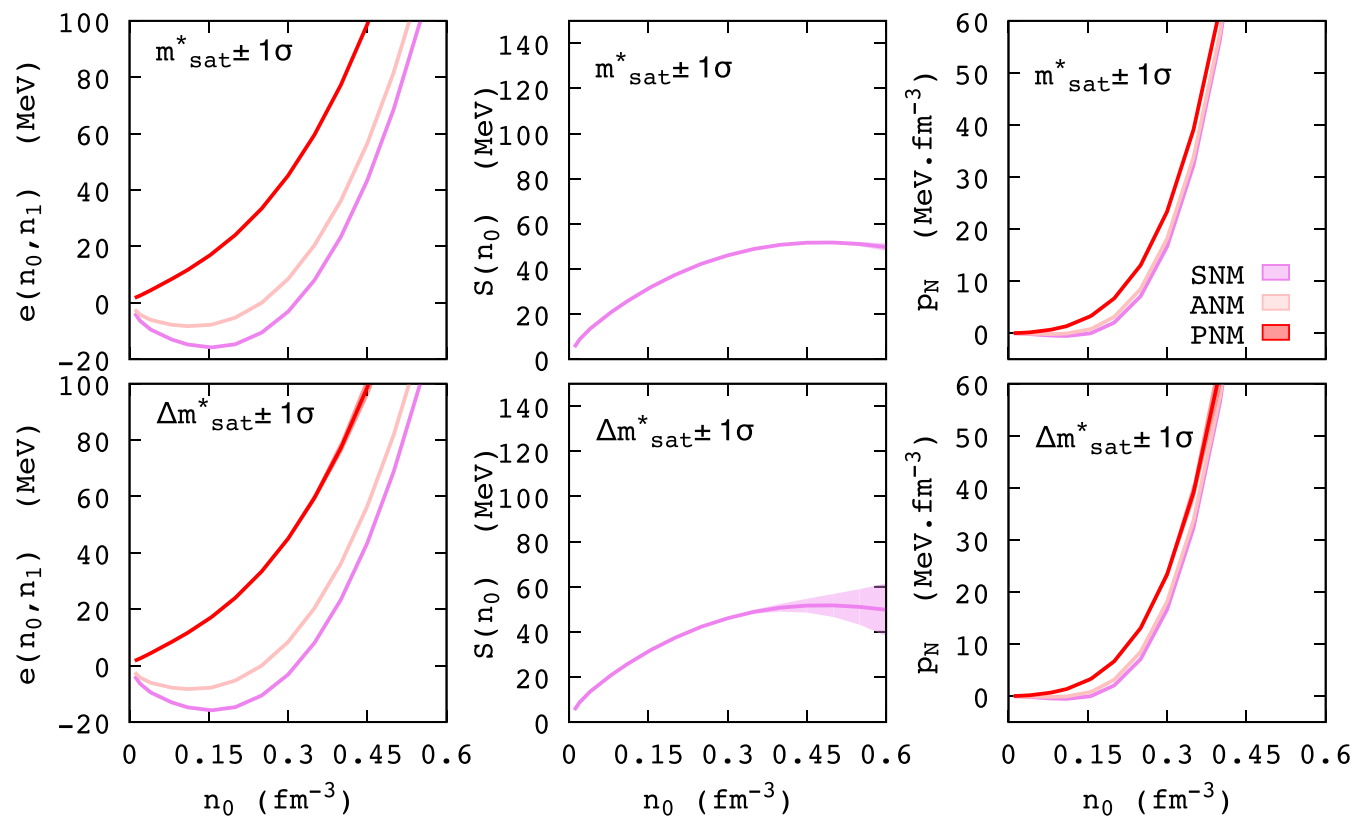

FIG. 16. Same as Fig. 11 for $m_{\text {sat }}^{*}$ (top panel) and $\Delta m_{\text {sat }}^{*}$ (bottom panel).

and the density of state, as well as the temperature dependence of the EOS. This effect will be studied in a future work.

\section{B. Global effect varying isoscalar and isovector empirical parameters}

We now discuss globally the impact of the isoscalar and isovector empirical parameters. They are varied independently all together within their own uncertainties, and the impact is shown in Fig. 17 (for the isoscalar empirical parameters) and in Fig. 18 (for the isovector empirical parameters).
Looking at Figs. 17 and 18, one has the impression that, in spite of the tremendous effort of the community in recent years, the EOS above $2-3 n_{\text {sat }}$ is completely unknown. This impression is, however, not fully correct for at least two reasons. We have seen in the preceding sections that the meta-EOS is sufficiently flexible to explore all possible density dependencies, thus being able to reproduce all existing models. The drawback of this flexibility is that it can also allow unphysical parametrizations. This can be clearly seen in Figs. 17 and 18: Models with negative pressures or negative symmetry energies can be explored by the meta-EOS but are indeed
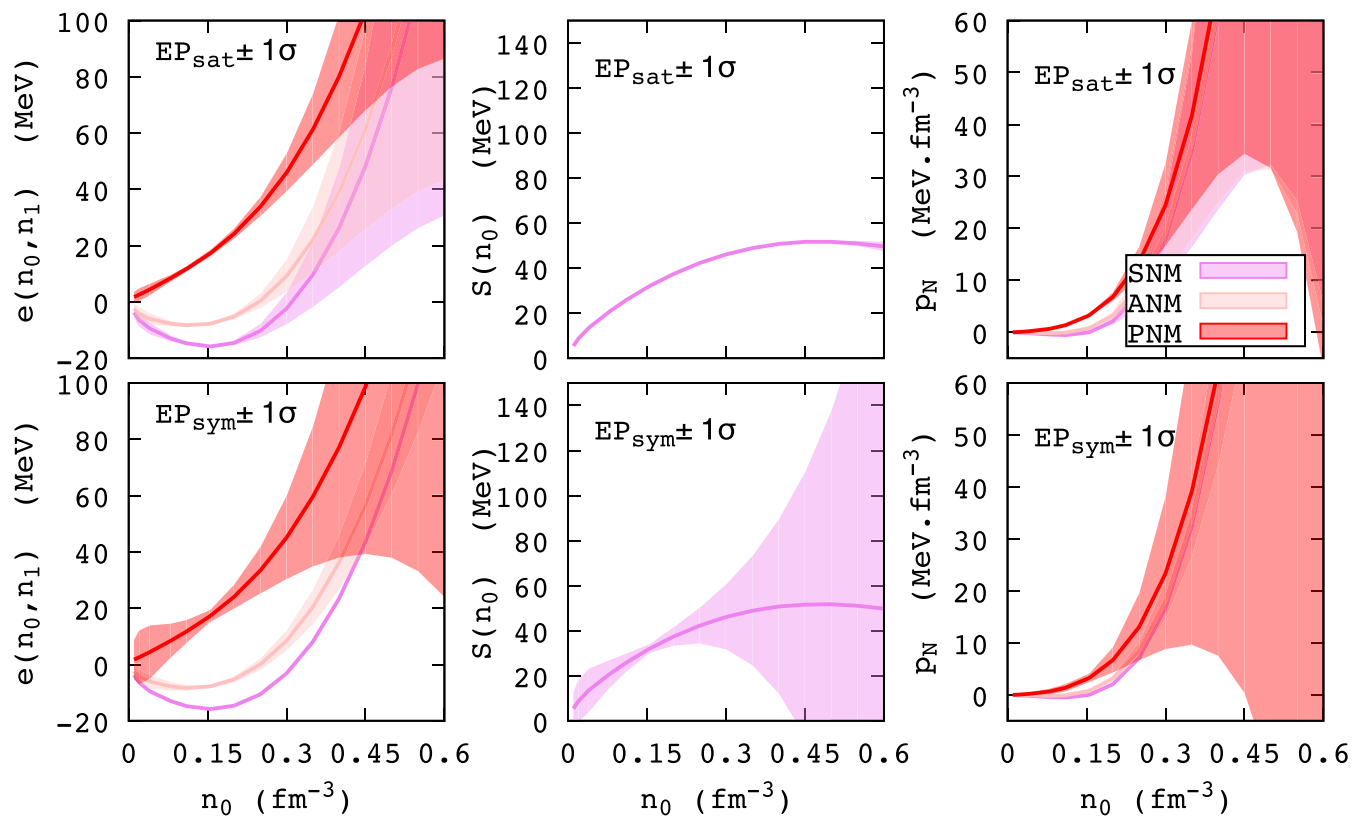

FIG. 17. Effect of varying the value of all empirical parameters in the isoscalar (top panels) and isovector (bottom panels) channels. From left to right: energy per nucleon, symmetry energy, and pressure in $\operatorname{SNM}(\delta=0), \operatorname{ANM}(\delta=0.5)$, and $\operatorname{PNM}(\delta=1)$. 

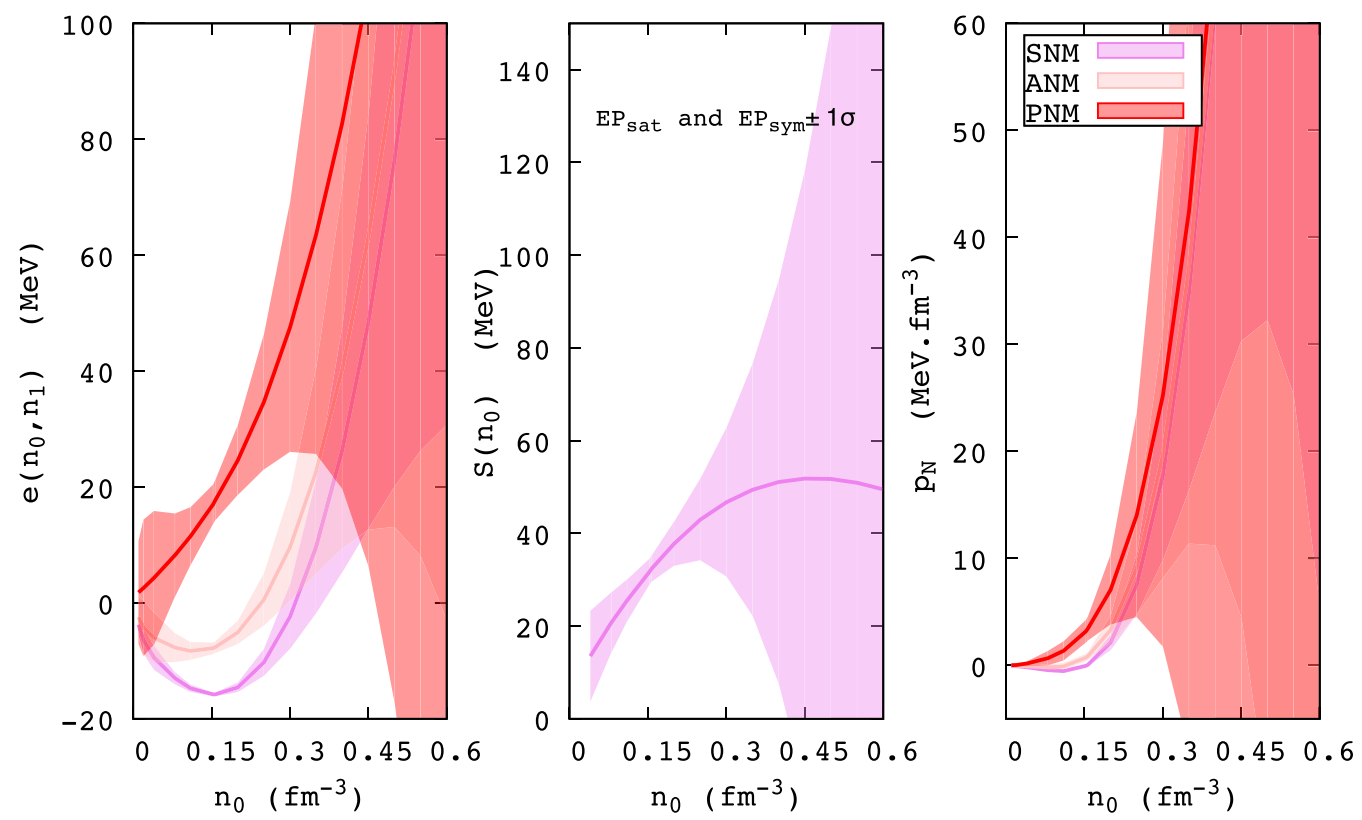

FIG. 18. Same as Fig. 17, varying the value of the empirical parameters all together.

forbidden by the stability requirement. The speed of sound is not represented in these figures, but superluminal models also belong to our possible parameter sets. It is clear that these unphysical models have to be excluded before reasonable error bars on the equation of state can be calculated.

The second reason why the bands of Figs. 17 and 18 cannot be interpreted as error bars on the EOS is that by construction the different parameters of the meta-EOS are independent. This is a quality of the model, because it allows us to do sensitivity studies and it avoids spurious correlations due to a too-limited number of parameters in existing functionals. However, physical correlations among the empirical parameters can exist, like the ones shown by the $a b$ initio calculation in Fig. 9. These correlations limit the parameter space and therefore the uncertainty intervals on the EOS. Such correlations can only be found if the ensemble of metaEOS is filtered through the requirement of reproduction of experimental or observational data. This statistical analysis of the meta-EOS can be performed with standard Bayesian techniques and is the object of a forthcoming paper [43].

\section{CONCLUSIONS}

In this paper, we have presented a meta-EOS for uniform matter which is very simply related to the empirical parameters characterizing the density and isospin density dependence of the EOS. This meta-EOS is based on nucleonic degrees of freedom and assumes that they are nonrelativistic. It allows a natural implementation of our best knowledge of the nuclear empirical parameters which is based on nuclear physics experiments and which we have discussed in some detail.

We have analyzed the confidence intervals of the empirical parameters obtained with different methods, namely from the direct analysis of experimental data and from a statistical analysis of various theoretical modelings. Phenomenological and $a b$ initio approaches, as well as relativistic and nonrela- tivistic interactions, have been analyzed in detail, and from this study, we have proposed a set of average values and estimated uncertainties for all empirical parameters from $E_{\text {sat/sym }}$ up to the kurtosis ones $Z_{\text {sat/sym }}$; see Table VII.

Finally, we have analyzed the impact of the uncertainties on the empirical parameters on the meta-EOS of nucleonic matter. We have deduced that the lowest order empirical parameters which require better determination in the future are the skewness parameter $Q_{\text {sat }}$ in the isoscalar channel, and the slope of the symmetry energy $L_{\text {sym }}$ and its curvature $K_{\text {sym }}$ in the isovector channel. The determination of these parameters needs to depart substantially from saturation density, either below or above. They could be determined either by relativistic heavy-ion collision; see, for instance, Ref. [133], or as we already discussed by the mass and radii constraints of NS [20-26]. The observation of the gravitational waves coming from NS merging are also expected to give tight constraints on the properties of dense matter [134]. Most probably, it is a combination of these different constraints which will be able to provide a better knowledge on these empirical parameters.

We have also discussed the density dependence of the symmetry energy which is provided by our meta-EOS, and we have shown that a rich behavior of the symmetry energy below and above $n_{\text {sat }}$ is possible by combining both $L_{\text {sym }}$ and $K_{\text {sym }}$. If $K_{\text {sym }}$ is negligible, $L_{\text {sym }}$ alone provides the well-known isosoft (below) and isostiff (above) or vice versa, while if $K_{\text {sym }}$ is large enough, different behaviors can be generated: isosoft (below) and isosoft (above) or isostiff (below) and isostiff (above).

The meta-EOS which is presented in this paper is an example of possible metamodeling. Further extensions are possible, such as implementing quartic dependence in the asymmetry parameter, considering relativistic kinetic energies instead of nonrelativistic, or replacing the polynomial expansion in density by an expansion in Fermi momentum or other quantity. Considering the present meta-EOS, it will be applied to $\beta$-equilibrium matter in neutron stars in a 
forthcoming paper [43]. In the future, we want also to use this meta-EOS as a density functional and apply it to the determination of the global structure of finite nuclei [135]. In our future application of this meta-EOS, its simple relation to the empirical parameters is expected to easily highlight the role of the empirical parameters in the nuclear properties. Empirical parameters are indeed a simple way to encode the basic properties of nuclear matter and provides a link between nuclear physics experiments and astrophysical applications. They therefore constitute an interesting and promising tool for better synergy between nuclear physics and astrophysics.
Finally, the interesting aspect of this meta-EOS is its simplicity and richness. Since it is simple, it shall be easy to implement it in many different modelings, going from the global structure of finite nuclei to more complex dynamical simulations. For neutron star physics, it offers the possibility of providing a simple approach which can encode many existing EOS for uniform matter, as well as new EOS, which properties could be tested against observations. It is therefore an interesting theoretical tool which could bring more consistency in the exploration of the nuclear EOS from different branches of nuclear physics and astrophysics.

TABLE X. Empirical properties of nuclear matter for Skyrme-type interactions.

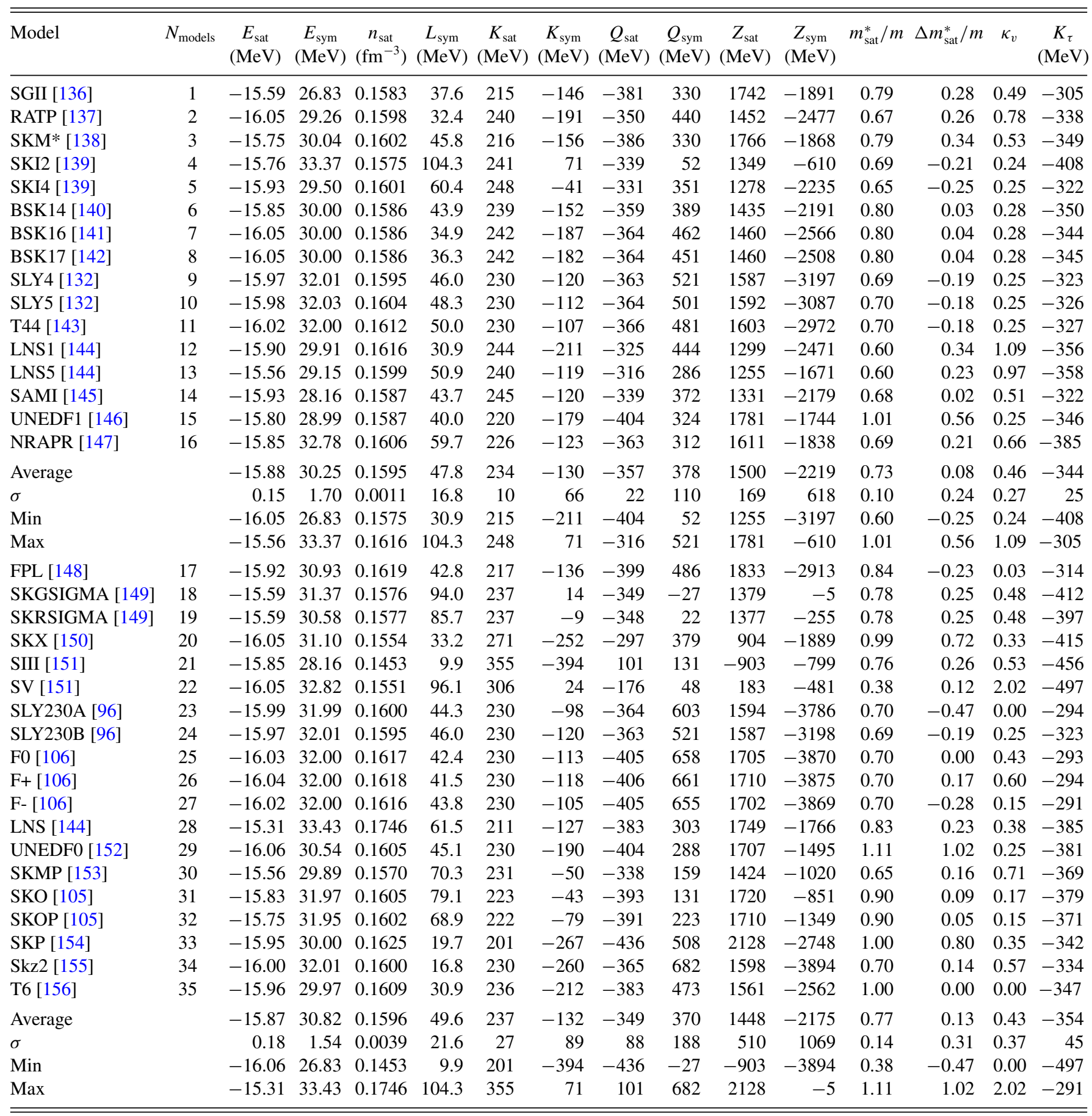


TABLE XI. Same as Table X for RMF models.

\begin{tabular}{|c|c|c|c|c|c|c|c|c|c|c|c|c|c|c|c|}
\hline DDME1 [69] & 36 & -16.20 & 33.07 & 0.1520 & 55.5 & 245 & -101 & 317 & 705 & 4867 & -5717 & 0.66 & -0.06 & 0.45 & -506 \\
\hline $\operatorname{DDME} \delta$ [157] & 38 & -16.12 & 32.35 & 0.1520 & 52.8 & 219 & -118 & -748 & 846 & 3950 & -3545 & 0.69 & -0.17 & 0.27 & -255 \\
\hline NL3 [87] & 39 & -16.24 & 37.35 & 0.1480 & 118.3 & 271 & 101 & 198 & 182 & 9302 & -3961 & 0.67 & -0.08 & 0.40 & -696 \\
\hline NL3s [67] & 40 & -16.31 & 38.71 & 0.1500 & 122.7 & 259 & 106 & 124 & 224 & 9997 & -3920 & 0.67 & -0.09 & 0.39 & -690 \\
\hline PK1 [68] & 42 & -16.27 & 37.59 & 0.1480 & 115.7 & 282 & 55 & -29 & -86 & 4008 & -2866 & 0.68 & -0.08 & 0.38 & -627 \\
\hline PK1R [68] & 43 & -16.27 & 37.78 & 0.1480 & 116.3 & 283 & 56 & -21 & -86 & 4032 & -2902 & 0.68 & -0.08 & 0.38 & -634 \\
\hline PKDD [68] & 44 & -16.27 & 31.19 & 0.1495 & 79.5 & 261 & -50 & -119 & -28 & 4213 & -1315 & 0.65 & -0.08 & 0.44 & -491 \\
\hline TM1 [159] & 45 & -16.26 & 36.94 & 0.1450 & 111.0 & 281 & 34 & -285 & -67 & 2014 & -1546 & 0.71 & -0.09 & 0.32 & -520 \\
\hline TW99 [160] & 46 & -16.25 & 32.77 & 0.1530 & 55.3 & 240 & -125 & -540 & 539 & 3749 & -3307 & 0.64 & -0.06 & 0.49 & -332 \\
\hline Average & & -16.24 & 35.11 & 0.1494 & 90.2 & 268 & -5 & -2 & 271 & 5058 & -3672 & 0.67 & -0.08 & 0.40 & -549 \\
\hline
\end{tabular}

\section{ACKNOWLEDGMENTS}

This work was partially supported by $\mathrm{CNPq}$ (Brazil), Processes No. 209440/2013-9 and No. 400877/2015-5, by the SN2NS Project No. ANR-10-BLAN-0503, and by NewCompStar COST Action No. MP1304. J.M. thanks N. Baillot, G. Bertsch, K. Bennaceur, A. Bulgac, G. Colò, Sébastien Guillot, and J. Meyer for stimulating discussions and interesting suggestions during the completion of this work. J.M. thanks Ingo Tews as well for providing him the data for the energy per nucleon and unpublished pressure from his work [11] and J.-J. Li for providing tables of empirical parameters for relativistic approaches.

\section{APPENDIX: DETAILED TABLES OF EMPIRICAL PARAMETERS}

In this Appendix, we provide more details on the general analysis of the empirical parameters deduced from nuclear interactions or effective Lagrangians.

The empirical parameters provided by Skyrme interactions are given in Table $\mathrm{X}$. This table is separated into two parts, the first 16 Skyrme models and the others. The reason for this separation is explain in Sec. IIB. We briefly summarize it: The first 16 Skyrme models have been selected since they are widely used interactions. In addition, we have limited the number of models per groups generating these interactions in order to mix as much as possible the various assumptions in the fitting protocols. The other Skyrme interactions, from 17th to 35 th, are also Skyrme interactions widely used either in finite nuclei (for most of them) or in nuclear matter. They are there to test the sensitivity of the statistical analysis based on the first 16 .

In Table XI, we have listed the RMF effective Lagrangians that we have studied in this paper. Let us note that the effective mass reported in this table is the Landau (nonrelativistic) one deduced from the momentum dependence of the nonrelativistic energy density.

Finally, we list in Table XII a few of RHF effective Lagrangians. The small number of models in this table is due to the very recent development of such approaches. It shall be remarked that these effective Lagrangians have been determined by a single group, and it would be interesting in the future to see more of these modelings.

In Table XIII are shown the empirical parameters $Q_{\text {sat/sym }}$ and $Z_{\text {sat/sym }}$ which are obtained from the meta-EOS ELFd. The standard deviation in the energy per nucleon in symmetric matter $\sigma_{e}(\mathrm{SM})$ and in neutron matter $\sigma_{e}(\mathrm{NM})$ between ELFd

TABLE XII. Same as Table X for RHF models.

\begin{tabular}{|c|c|c|c|c|c|c|c|c|c|c|c|c|c|c|c|}
\hline Model & $N_{\text {models }}$ & $\begin{array}{c}E_{\text {sat }} \\
(\mathrm{MeV})\end{array}$ & $\begin{array}{c}E_{\text {sym }} \\
(\mathrm{MeV})\end{array}$ & $\begin{array}{c}n_{\mathrm{sat}} \\
\left(\mathrm{fm}^{-3}\right)\end{array}$ & $\begin{array}{c}L_{\text {sym }} \\
(\mathrm{MeV})\end{array}$ & $\begin{array}{c}K_{\text {sat }} \\
(\mathrm{MeV})\end{array}$ & $\begin{array}{c}K_{\text {sym }} \\
(\mathrm{MeV})\end{array}$ & $\begin{array}{c}Q_{\text {sat }} \\
(\mathrm{MeV})\end{array}$ & $\begin{array}{c}Q_{\text {sym }} \\
(\mathrm{MeV})\end{array}$ & $\begin{array}{c}Z_{\text {sat }} \\
(\mathrm{MeV})\end{array}$ & $\begin{array}{c}Z_{\text {sym }} \\
(\mathrm{MeV})\end{array}$ & $m_{\text {sat }}^{*} / m$ & $\Delta m_{\mathrm{sat}}^{*} / m$ & $\kappa_{v}$ & $\begin{array}{c}K_{\tau} \\
(\mathrm{MeV})\end{array}$ \\
\hline PKA1 [161] & 47 & -15.83 & 36.02 & 0.1600 & 103.5 & 230 & 213 & 950 & 292 & 4935 & -16916 & 0.68 & -0.02 & 0.45 & -835 \\
\hline PKO2 [125] & 49 & -16.03 & 32.49 & 0.1510 & 75.9 & 250 & 77 & -10 & 821 & 6703 & -7993 & 0.76 & -0.02 & 0.30 & -375 \\
\hline PKO3 [125] & 50 & -16.04 & 32.99 & 0.1530 & 83.0 & 262 & 116 & 355 & 690 & 4581 & -8921 & 0.76 & -0.03 & 0.29 & -494 \\
\hline Min & & -16.04 & 32.49 & 0.1510 & 75.9 & 230 & 77 & -10 & 290 & 4581 & -16916 & 0.68 & -0.03 & 0.29 & -835 \\
\hline Max & & -15.83 & 36.02 & 0.1600 & 103.5 & 262 & 213 & 950 & 821 & 6703 & -5993 & 0.76 & -0.02 & 0.45 & -375 \\
\hline
\end{tabular}


TABLE XIII. Modification of the empirical parameters, $Q_{\text {sat } / \text { sym }}$ and $Z_{\text {sat/sym }}$, in the model ELFd adjusted to reproduce reference models.

\begin{tabular}{|c|c|c|c|c|c|c|c|c|c|}
\hline Model & $\begin{array}{c}Q_{\text {sat }} \\
(\mathrm{MeV})\end{array}$ & $\begin{array}{c}Q_{\text {sym }} \\
(\mathrm{MeV})\end{array}$ & $\begin{array}{c}Q_{\text {sym }} \\
\left(v_{4}=0\right) \\
(\mathrm{MeV})\end{array}$ & $\begin{array}{c}Z_{\text {sat }} \\
(\mathrm{MeV})\end{array}$ & $\begin{array}{c}Z_{\text {sym }} \\
(\mathrm{MeV})\end{array}$ & $\begin{array}{c}Z_{\text {sym }} \\
\left(v_{4}=0\right) \\
(\mathrm{MeV})\end{array}$ & $\sigma_{e}(\mathrm{SM})$ & $\sigma_{e}(\mathrm{NM})$ & $\begin{array}{l}\sigma_{e}(\mathrm{NM}) \\
\left(v_{4}=0\right)\end{array}$ \\
\hline RATP [137] & -222.18 & 347.83 & 279.10 & -414.59 & -1126.54 & -851.61 & 0.24 & 0.07 & 0.58 \\
\hline $\mathrm{SKM}^{*}[138]$ & -228.35 & 277.66 & 239.32 & -542.05 & -1097.83 & -944.48 & 0.29 & 0.19 & 0.24 \\
\hline SKI2 [139] & -214.11 & 25.49 & 5.56 & -447.81 & -223.15 & -143.41 & 0.23 & 0.18 & 0.10 \\
\hline BSK14 [140] & -217.69 & 297.78 & 227.08 & -578.08 & -884.03 & -601.20 & 0.26 & 0.09 & 0.58 \\
\hline BSK16 [141] & -221.14 & 350.01 & 262.83 & -573.66 & -952.84 & -604.13 & 0.26 & 0.05 & 0.76 \\
\hline BSK17 [142] & -221.14 & 341.95 & 257.21 & -573.68 & -941.63 & -602.66 & 0.26 & 0.06 & 0.74 \\
\hline SLY4 [132] & -225.01 & 350.68 & 226.01 & -443.11 & -690.35 & -191.68 & 0.26 & 0.05 & 1.19 \\
\hline SLY5 [132] & -223.62 & 337.37 & 217.90 & -451.22 & -679.62 & -201.75 & 0.25 & 0.05 & 1.13 \\
\hline DDME1 [69] & 564.80 & 328.92 & 263.14 & -2377.81 & -1297.60 & -1034.45 & 0.62 & 0.81 & 1.38 \\
\hline NL-SH [158] & 965.63 & -472.62 & -207.75 & -3542.60 & 52.43 & -1007.05 & 1.36 & 1.21 & 1.46 \\
\hline PK1 [68] & 240.19 & -316.97 & -125.65 & -2007.56 & -250.93 & -1016.22 & 0.57 & 0.70 & 1.40 \\
\hline PK1R [68] & 249.68 & -320.53 & -127.58 & -2031.73 & -244.39 & -1016.22 & 0.58 & 0.72 & 1.40 \\
\hline PKDD [68] & 175.33 & 125.02 & 95.48 & -1556.55 & -1141.26 & -1023.08 & 0.34 & 4.69 & 4.82 \\
\hline TM1 [159] & -105.20 & -187.08 & -51.79 & -1188.79 & -461.28 & -1002.45 & 0.19 & 0.31 & 1.43 \\
\hline TW99 [160] & -206.47 & 400.18 & 317.66 & -729.57 & -1369.06 & -1038.98 & 0.56 & 0.46 & 0.52 \\
\hline PKA1 [125] & 877.58 & -1176.76 & -584.77 & -3249.25 & 1297.52 & -1070.43 & 1.74 & 1.05 & 5.72 \\
\hline PKO1 [125] & 518.91 & -489.42 & -238.03 & -2342.01 & -28.93 & -1034.45 & 0.62 & 0.41 & 2.35 \\
\hline PKO2 [125] & 432.64 & -169.80 & -85.68 & -2088.47 & -693.41 & -1029.91 & 0.55 & 0.42 & 0.64 \\
\hline PKO3 [125] & 595.24 & -424.75 & -216.59 & -2533.23 & -206.36 & -1038.98 & 0.72 & 0.63 & 1.68 \\
\hline
\end{tabular}

and the original prediction from each of the listed models are also given, showing the very good agreement between the meta-EOS ELFd and the original interaction.

The number of parameters in the meta-EOS, including the effective mass parameters, is 12 . It could be interesting to perform further analysis to reduce this number a bit. For this purpose, we have explored the impact of removing the highest order isovector empirical parameter, by setting $v_{4}^{\text {iv }}=0$, on the comparison between the EOS ELFd and the original EOS. The results are given in Table XIII. It is clear that the impact is extremely small, showing that this parameter has a weak influence on the EOS below $4 n_{\text {sat }}$.
[1] S. J. Bell and A. Hewish, Nature (London) 215, 25 (1967)

[2] A. Hewish, S. J. Bell, J. D. H. Pilkington, P. F. Scott, and R. A. Collins, Nature (London) 224, 472 (1969).

[3] A. Hewish, S. J. Bell, J. D. H. Pilkington, P. F. Scott, and R. A. Collins, Nature (London) 217, 709 (1968).

[4] R. C. Tolman, Phys. Rev. 55, 364 (1939).

[5] J. R. Oppenheimer and G. M. Volkoff, Phys. Rev. 55, 374 (1939).

[6] J. M. Lattimer and M. Prakash, Phys. Rev. Lett. 94, 111101 (2005).

[7] P. B. Demorest, T. Pennucci, S. M. Ransom, M. S. E. Roberts, and J. W. T. Hessels, Nature (London) 467, 1081 (2010).

[8] J. Antoniadis, P. C. C. Freire, N. Wex, T. M. Tauris, R. S. Lynch, M. H. van Kerkwijk, M. Kramer, C. Bassa, V. S. Dhillon, T. Driebe et al., Science 340, 1233232 (2013).

[9] M. Baldo, A. Polls, A. Rios, H.-J. Schulze, and I. Vidaña, Phys. Rev. C 86, 064001 (2012).

[10] S. Gandolfi, J. Carlson, S. Reddy, A. W. Steiner, and R. B. Wiringa, Eur. Phys. J. A 50, 10 (2014).
[11] I. Tews, T. Krüger, K. Hebeler, and A. Schwenk, Phys. Rev. Lett. 110, 032504 (2013).

[12] C. Drischler, K. Hebeler, and A. Schwenk, Phys. Rev. C 93, 054314 (2016).

[13] A. Roggero, A. Mukherjee, and F. Pederiva, Phys. Rev. Lett. 112, 221103 (2014).

[14] G. Wlazłowski, J. W. Holt, S. Moroz, A. Bulgac, and K. J. Roche, Phys. Rev. Lett. 113, 182503 (2014).

[15] J. E. Lynn, I. Tews, J. Carlson, S. Gandolfi, A. Gezerlis, K. E. Schmidt, and A. Schwenk, arXiv:1606.07668.

[16] K. Hebeler, J. M. Lattimer, C. J. Pethick, and A. Schwenk, Astrophys. J. 773, 11 (2013).

[17] I. Tews, Phys. Rev. C 95, 015803 (2017).

[18] M. Dutra, O. Lourenço, J. S. Sá Martins, A. Delfino, J. R. Stone, and P. D. Stevenson, Phys. Rev. C 85, 035201 (2012).

[19] J. S. Read, B. D. Lackey, B. J. Owen, and J. L. Friedman, Phys. Rev. D 79, 124032 (2009).

[20] F. Özel and D. Psaltis, Phys. Rev. D 80, 103003 (2009). 
[21] F. Özel, G. Baym, and T. Güver, Phys. Rev. D 82, 101301(R) (2010).

[22] A. W. Steiner, J. M. Lattimer, and E. F. Brown, Astrophys. J. 722, 33 (2010)

[23] S. Guillot, R. E. Rutledge, and E. F. Brown, Astrophys. J. 732, 88 (2011)

[24] S. Guillot, M. Servillat, N. Webb, and R. E. Rutledge, Astrophys. J. 772, 7 (2013).

[25] A. W. Steiner, J. M. Lattimer, and E. F. Brown, Astrophys. J. 765, L5 (2013).

[26] J. M. Lattimer and A. W. Steiner, Astrophys. J. 784, 123 (2014).

[27] S. Guillot and R. E. Rutledge, Astrophys. J. 796, L3 (2014).

[28] S. Guillot, Mem. S. A. It. 87, 521 (2016).

[29] C. A. Raithel, F. Özel, and D. Psaltis, Astrophys. J. 831, 44 (2016).

[30] C. A. Raithel, F. Özel, and D. Psaltis, Astrophys. J. 844, 156 (2017).

[31] N. Alam, B. K. Agrawal, J. N. De, S. K. Samaddar, and G. Colò, Phys. Rev. C 90, 054317 (2014).

[32] E. Khan, J. Margueron, and I. Vidaña, Phys. Rev. Lett. 109, 092501 (2012); E. Khan and J. Margueron, Phys. Rev. C 88, 034319 (2013).

[33] E. Khan, Phys. Scr. T 152, 014008 (2013).

[34] A. Roggero, A. Mukherjee, and F. Pederiva, Phys. Rev. C 92, 054303 (2015)

[35] E. Rrapaj, A. Roggero, and J. W. Holt, Phys. Rev. C 93, 065801 (2016).

[36] B. M. Santos, M. Dutra, O. Lourenço, and A. Delfino, Phys. Rev. C 92, 015210 (2015)

[37] Ch. C. Moustakidis, T. Gaitanos, Ch. Margaritis, and G. A. Lalazissis, Phys. Rev. C 95, 045801 (2017).

[38] A. Bulgac, M. M. Forbes, and S. Jin, arXiv:1506.09195.

[39] J. P. Kleijnen, Statistical Tools for Simulation Practitioners (Marcel Dekker Inc., New York, 1987), Vol. 73.

[40] T. W. Simpson, J. D. Peplinski, P. N. Koch, J. K. Allen, Eng. Comput. 17, 129 (2001).

[41] R. Jin, X. Du, and W. Chen, Struct. Multidiscip. Optim. 25, 99 (2003).

[42] Y. Jin, Soft Comput. 9, 3 (2005).

[43] J. Margueron, R. Hoffmann Casali, and F. Gulminelli, Phys. Rev. C 97, 025806 (2018).

[44] J. Piekarewicz and M. Centelles, Phys. Rev. C 79, 054311 (2009).

[45] C. Ducoin, J. Margueron, and C. Providência, Europhys. Lett. 91, 32001 (2010).

[46] C. Ducoin, J. Margueron, C. Providência, and I. Vidaña, Phys. Rev. C 83, 045810 (2011).

[47] W. D. Myers and W. J. Swiatecki, Ann. Phys. 55, 395 (1969).

[48] P.-G. Reinhard, M. Bender, W. Nazarewicz, and T. Vertse, Phys. Rev. C 73, 014309 (2006).

[49] B.-J. Cai and L.-W. Chen, Phys. Rev. C 85, 024302 (2012).

[50] C. C. Moustakidis, Phys. Rev. C 86, 015801 (2012).

[51] W. M. Seif and D. N. Basu, Phys. Rev. C 89, 028801 (2014).

[52] W.Zuo, A. Lejeune, U. Lombardo, and J.-F. Mathiot, Eur. Phys. J. A 14, 469 (2002).

[53] I. Vidaña, C. Providência, A. Polls, and A. Rios, Phys. Rev. C 80, 045806 (2009).

[54] Y. Tsukioka and H. Nakada, Prog. Theor. Exp. Phys. 073D02 (2017).

[55] C. S. Wang, K. C. Chung, and A. J. Santiago, Phys. Rev. C 60, 034310 (1999).
[56] W. D. Myers and W. J. Swiatecki, Nucl. Phys. 81, 1 (1966).

[57] G. Royer, Nucl. Phys. A 807, 105 (2008).

[58] K. Pomorski and J. Dudek, Phys. Rev. C 67, 044316 (2003).

[59] W. D. Myers, Droplet Model of Atomic Nuclei (Springer, Berlin, 1977).

[60] F. Buchinger, J. E. Crawford, A. K. Dutta, J. M. Pearson, and F. Tondeur, Phys. Rev. C 49, 1402 (1994).

[61] L. Satpathy, V. S. U. Maheswari, and R. C. Nayak, Phys. Rep. 319, 85 (1999).

[62] F. Tondeur, D. Berdichevsky, and M. Farine, Z. Phys. A 325, 405 (1986).

[63] P. Klüpfel, P.-G. Reinhard, T. J. Bürvenich, and J. A. Maruhn, Phys. Rev. C 79, 034310 (2009).

[64] S. Goriely, M. Samyn, P.-H. Heenen, J. M. Pearson, and F. Tondeur, Phys. Rev. C 66, 024326 (2002).

[65] S. Goriely, Nucl. Phys. A 933, 68 (2015).

[66] J. D. McDonnell, N. Schunck, D. Higdon, J. Sarich, S. M. Wild, and W. Nazarewicz, Phys. Rev. Lett. 114, 122501 (2015).

[67] G. A. Lalazissis, S. Karatzikos, R. Fossion, D. Pena-Arteaga, A. V. Afanasjev, and P. Ring, Phys. Lett. B 671, 36 (2009).

[68] W. Long, J. Meng, N. Van Giai, and S. G. Zhou, Phys. Rev. C 69, 034319 (2004).

[69] T. Nikšić, D. Vretenar, P. Finelli, and P. Ring, Phys. Rev. C 66, 024306 (2002).

[70] G. A. Lalazissis, T. Nikšić, D. Vretenar, and P. Ring, Phys. Rev. C 71, 024312 (2005).

[71] D. Berdichevsky, R. Fleming, D. W. L. Sprung, and F. Tondeur, Z. Phys. A 329, 393 (1988).

[72] M. Farine, J. M. Pearson, and F. Tondeur, Nucl. Phys. A 615, 135 (1997).

[73] M. Centelles, X. Roca-Maza, X. Viñas, and M. Warda, Phys. Rev. Lett. 102, 122502 (2009).

[74] A. Carbone, G. Colò, A. Bracco, L.-G. Cao, P. F. Bortignon, F. Camera, and O. Wieland, Phys. Rev. C 81, 041301 (2010).

[75] P. Danielewicz and J. Lee, Nucl. Phys. A 922, 1 (2014).

[76] W. G. Newton, J. Hooker, M. Gearheart, K. Murphy, D.-H. Wen, F. J. Fattoyev, and B.-A. Li, Eur. Phys. J. A 50, 41 (2014).

[77] J. M. Lattimer and A. W. Steiner, Eur. Phys. J. A 50, 40 (2014).

[78] H. Sagawa, S. Yoshida, G.-M. Zeng, J.-Z. Gu, and X.-Z. Zhang, Phys. Rev. C 76, 034327 (2007).

[79] D. Patel, U. Garg, M. Itoh, H. Akimune, G. P. A. Berg, M. Fujiwara, M. N. Harakeh, C. Iwamoto, T. Kawabata, K. Kawase, J. T. Matta, T. Murakami, A. Okamoto, T. Sako, K. W. Schlax, F. Takahashi, M. White, and M. Yosoi, Phys. Lett. B 735, 387 (2014).

[80] S. Abrahamyan et al. (PREX Collaboration), Phys. Rev. Lett. 108, 112502 (2012).

[81] G. F. Bertsch and D. Bingham, Phys. Rev. Lett. 119, 252501 (2017).

[82] P. Haensel, M. Kutschera, and M. Proszynski, Astron. Astrophys. 102, 299 (1981).

[83] J. R. Stone, N. J. Stone, and S. A. Moszkowski, Phys. Rev. C 89, 044316 (2014).

[84] J.-P. Blaizot, Phys. Rep. 64, 171 (1980).

[85] J.-P. Blaizot, J.-F. Berger, J. Dechargé, and M. Girod, Nucl. Phys. A 591, 435 (1995).

[86] M. Baldo, L. M. Robledo, P. Schuck, and X. Viñas, Phys. Rev. C 87, 064305 (2013). 
[87] G. A. Lalazissis, J. König, and P. Ring, Phys. Rev. C 55, 540 (1997).

[88] Z. Yu Ma, N. V. Giai, A. Wandelt, D. Vretenar, and P. Ring, Nucl. Phys. A 686, 173 (2001).

[89] D. Vretenar, T. Nikšić, and P. Ring, Phys. Rev. C 68, 024310 (2003).

[90] S. Shlomo and A. I. Sanzhur, Phys. Rev. C 65, 044310 (2002).

[91] G. Colò, N. V. Giai, J. Meyer, K. Bennaceur, and P. Bonche, Phys. Rev. C 70, 024307 (2004).

[92] G. Colò, H. Sagawa, S. Fracasso, and P. F. Bortignon, Phys. Lett. B 668, 457 (2008).

[93] X. Roca-Maza, M. Brenna, B. K. Agrawal, P. F. Bortignon, G. Colò, L. G. Cao, N. Paar, and D. Vretenar, Phys. Rev. C 87, 034301 (2013).

[94] L. Trippa, G. Colò, and E. Vigezzi, Phys. Rev. C 77, 061304(R) (2008).

[95] F. Aymard, F. Gulminelli, and J. Margueron, Phys. Rev. C 89, 065807 (2014).

[96] E. Chabanat, P. Bonche, P. Haensel, J. Meyer, and R. Schaeffer, Nucl. Phys. A 627, 710 (1997).

[97] M. Bender, P.-H. Heenen, and P.-G. Reinhard, Rev. Mod. Phys. 75, 121 (2003).

[98] B.-A. Li, L.-W. Chen, and C. Ming, Phys. Rep. 464, 113 (2008).

[99] P. Danielewicz and J. Lee, Nucl. Phys. A 818, 36 (2009).

[100] B.-A. Li, À. Ramos, G. Verde, and I. Vidaña, Eur. Phys. J. A 50, 9 (2014).

[101] B.-A. Li and X. Han, Phys. Lett. B 727, 276 (2013).

[102] I. Tews, J. M. Lattimer, A. Ohnishi, and E. E. Kolomeitsev, Astrophys. J. 848, 105 (2017).

[103] C. Constantinou, B. Muccioli, M. Prakash, and J. M. Lattimer, Phys. Rev. C 89, 065802 (2014).

[104] E. Lipparini and S. Stringari, Phys. Rep. 175, 103 (1989).

[105] P.-G. Reinhard, D. J. Dean, W. Nazarewicz, J. Dobaczewski, J. A. Maruhn, and M. R. Strayer, Phys. Rev. C 60, 014316 (1999).

[106] T. Lesinski, K. Bennaceur, T. Duguet, and J. Meyer, Phys. Rev. C 74, 044315 (2006).

[107] F. G. Perey and B. Buck, Nucl. Phys. 32, 353 (1962).

[108] Z. Y. Ma and J. Wambach, Nucl. Phys. A 402, 275 (1983).

[109] C. B. Dover and N. Van Giai, Nucl. Phys. 190, 373 (1972).

[110] K. S. A. Hassaneen and H. Müther, Phys. Rev. C 70, 054308 (2004).

[111] Z. Y. Ma, J. Rong, B. Q. Chen, Z. Y. Zhu, and H. Q. Song, Phys. Lett. B 604, 170 (2004).

[112] E. N. E. van Dalen, C. Fuchs, and A. Faessler, Phys. Rev. Lett. 95, 022302 (2005).

[113] G. E. Brown and J. H. Gunn, Nucl. Phys. 46, 598 (1963).

[114] G. F. Bertsch and T. T. S. Kuo, Nucl. Phys. A 112, 204 (1968).

[115] V. Bernard and N. Van Giai, Nucl. Phys. A 327, 397 (1979).

[116] G. E. Brown, J. S. Dehesa, and J. Speth, Nucl. Phys. A 330, 290 (1979).

[117] P. F. Bortignon and C. H. Dasso, Phys. Lett. B 189, 381 (1987).

[118] N. Van Giai and P. Van Thieu, Phys. Lett. B 126, 421 (1983).

[119] C. Mahaux, P. F. Bortignon, R. A. Broglia, and C. H. Dasso, Phys. Rep. 120, 1 (1985).

[120] C. Mahaux and R. Sartor, Phys. Rep. 211, 53 (1992).

[121] I. Bombaci and U. Lombardo, Phys. Rev. C 44, 1892 (1991).

[122] W. Zuo, I. Bombaci, and U. Lombardo, Phys. Rev. C 60, 024605 (1999).

[123] F. Hofmann, C. M. Keil, and H. Lenske, Phys. Rev. C 64, 034314 (2001).
[124] W. Satuła, R. A. Wyss, and M. Rafalski, Phys. Rev. C 74, 011301(R) (2006).

[125] W. Long, N. Van Giai, and J. Meng, Phys. Lett. B 640, 150 (2006).

[126] S. Gandolfi, J. Carlson, and S. Reddy, Phys. Rev. C 85, 032801(R) (2012).

[127] X. Zhang and M. Prakash, Phys. Rev. C 93, 055805 (2016).

[128] B. D. Serot and J. D. Walecka, Int. J. Mod. Phys. E 6, 515 (1997).

[129] J. Margueron, E. N. E. van Dalen, and C. Fuchs, Phys. Rev. C 76, 034309 (2007).

[130] M. Baldo, C. Maieron, P. Schuck, and X. Viñas, Nucl. Phys. A 736, 241 (2004).

[131] M. Baldo, P. Schuck, and X. Viñas, Phys. Lett. B 663, 390 (2008).

[132] E. Chabanat, P. Bonche, P. Haensel, J. Meyer, and R. Schaeffer, Nucl. Phys. A 635, 231 (1997).

[133] P. Danielewicz, Nucl. Phys. A 685, 368c (2001).

[134] B. P. Abbott et al. (LIGO Scientific Collaboration and Virgo Collaboration), Phys. Rev. Lett. 119, 161101 (2017).

[135] D. Chatterjee, F. Gulminelli, Ad. R. Raduta, and J. Margueron, Phys. Rev. C 96, 065805 (2017).

[136] Nguyen Van Giai and H. Sagawa, Phys. Lett. B 106, 379 (1981).

[137] M. Rayet, M. Arnould, F. Tondeur, and G. Paulus, Astron. Astrophys. 116, 183 (1982).

[138] J. Bartel, P. Quentin, M. Brack, C. Guet, and H.-B. Hakansson, Nucl. Phys. A 386, 79 (1982).

[139] P.-G. Reinhard and H. Flocard, Nucl. Phys. A 584, 467 (1995).

[140] S. Goriely, M. Samyn, and J. M. Pearson, Phys. Rev. C 75, 064312 (2007).

[141] N. Chamel, S. Goriely, and J. M. Pearson, Nucl. Phys. A 812, 72 (2008).

[142] S. Goriely, N. Chamel, and J. M. Pearson, Phys. Rev. Lett. 102, 152503 (2009).

[143] T. Lesinski, M. Bender, K. Bennaceur, T. Duguet, and J. Meyer, Phys. Rev. C 76, 014312 (2007).

[144] L. G. Cao, U. Lombardo, C. W. Shen, and N. V. Giai, Phys. Rev. C 73, 014313 (2006).

[145] X. Roca-Maza, G. Colò, and H. Sagawa, Phys. Rev. C 86, 031306(R) (2012).

[146] M. Kortelainen, J. McDonnell, W. Nazarewicz, P.-G. Reinhard, J. Sarich, N. Schunck, M. V. Stoitsov, and S. M. Wild, Phys. Rev. C 85, 024304 (2012).

[147] A. W. Steiner, M. Prakash, J. M. Lattimer, and P. J. Ellis, Phys. Rep. 411, 325 (2005).

[148] J. Meyer, Lectures at the 11th Joliot-Curie School of Nuclear Physics, Maubuisson, September 1993 (unpublished).

[149] J. Friedrich and P.-G. Reinhard, Phys. Rev. C 33, 335 (1986)

[150] B. A. Brown, Phys. Rev. C 58, 220 (1998).

[151] M. Beiner, H. Flocard, N. Van Giai, and P. Quentin, Nucl. Phys. A 238, 29 (1975).

[152] M. Kortelainen, T. Lesinski, J. Moré, W. Nazarewicz, J. Sarich, N. Schunck, M. V. Stoitsov, and S. M. Wild, Phys. Rev. C 82, 024313 (2010).

[153] L. Bennour, P. H. Heenen, P. Bonche, J. Dobaczewski, and H. Flocard, Phys. Rev. C 40, 2834 (1989).

[154] J. Dobaczewski, H. Flocard, and J. Treiner, Nucl. Phys. A 422, 103 (1984).

[155] J. Margueron, J. Navarro, and N. V. Giai, Phys. Rev. C 66, 014303 (2002). 
[156] F. Tondeur, M. Brack, M. Farine, and J. M. Pearson, Nucl. Phys. A 420, 297 (1984).

[157] X. Roca-Maza, X. Viñas, M. Centelles, P. Ring, and P. Schuck, Phys. Rev. C 84, 054309 (2011).

[158] M. M. Sharma, M. A. Nagarajan, and P. Ring, Phys. Lett. B 312, 377 (1993).
[159] K. Sumiyoshi, H. Kuwabara, and H. Toki, Nucl. Phys. A 581, 725 (1995).

[160] S. Typel and H. H. Wolter, Nucl. Phys. A 656, 331 (1999).

[161] W. H. Long, H. Sagawa, N. V. Giai, and J. Meng, Phys. Rev. C 76, 034314 (2007). 